\title{
The topological trace formula
}

\author{
By Mark Goresky and Robert MacPherson at Princeton
}

\begin{abstract}
The topological trace formula is a computation of the Lefschetz number of a Hecke correspondence $C$ acting on the weighted cohomology groups, defined in $[\mathrm{GHM}]$, of a locally symmetric space $X$. It expresses this Lefschetz number as a sum of contributions from fixed point components of $C$ on the reductive Borel Serre compactification of $X$. The proof uses the Lefschetz fixed point formula of [GM2].
\end{abstract}

\section{Introduction}

1.1. The goal. Although this paper is self contained, it is actually the fourth in a series of five papers ([GM1], [GM2], $[\mathrm{GHM}]$, this paper, and [GKM]) in which we derive a formula for the Lefschetz number of a Hecke correspondence acting on the weighted cohomology groups of any locally symmetric space $X$. For various reasons, the publication of this paper was delayed for many years, and it is now appearing after [GKM], which logically depends on results from this paper.

In [GM1] the formula is described (without proof) for the special case of locally symmetric spaces associated to $\mathbf{S p}(4, \mathbb{R})$.

In [GM2] we address the general topological problem of determining the contribution from a single fixed point component to the Lefschetz number of an arbitrary "weakly hyperbolic" correspondence acting on a complex of sheaves on a compact stratified space. (An error [GM2] is corrected in $\S 11.7$ of the present paper.)

In $[\mathrm{GHM}]$ we construct a family of (complexes of ) sheaves $\mathbf{W}^{v} \mathbf{C}^{\bullet}(\bar{X}, \mathbf{E})$ on the reductive Borel Serre compactification $\bar{X}$ of the locally symmetric space $X$, with coefficients in a local system E. The (hyper) cohomology of this complex is the weighted cohomology $W^{v} H^{*}(\bar{X}, \mathbf{E})$. For various choices of $v$ the weighted cohomology may be identified with the ordinary cohomology $H^{*}(X, \mathbf{E})$, the compact support cohomology $H_{c}^{*}(X, \mathbf{E})$, the $L^{2}$ cohomology $H_{(2)}^{*}(X, \mathbf{E})$ (when it is finite dimensional), or with Franke's weighted $L^{2}$ cohomology $[\mathrm{F}],[\mathrm{N}]$. The goal is (a) to apply the formula of [GM2] to the action of a Hecke correspondence $C[g]$ on the weighted cohomology $W^{v} H^{*}(\bar{X}, \mathbf{E})$, and (b) to sum these contributions over all the fixed point components in $\bar{X}$, so as to obtain a formula for the Lefschetz trace of $C[g]$ on the weighted cohomology. 
For part (a) one must verify that each Hecke correspondence $C[g]$ is "weakly hyperbolic" in the sense of [GM2], and this is the first main result of the present paper, Theorem 11.9. In the process, we describe some of the rich geometry of Hecke correspondences and their fixed points. In Theorem 13.6, the second main result of this paper, we complete part (a) by describing the local contribution to the Lefschetz number in terms of roots and weights.

For part (b), one may sum the contributions from the fixed point components of $\bar{X}$ in either the adelic setting or the discrete group setting. This is accomplished in the adelic setting using orbital integrals, in [GKM], Theorem 7.14 (p. 535). Theorem 7.14 of [GKM] uses Theorem 13.6 of the present paper as its starting point: it occurs as the expression for $L_{Q}(\gamma)$ on page 534 of $[\mathrm{GKM}]$.

When the $L^{2}$ cohomology of $X$ is finite dimensional (the equal rank case), the Lefschetz number of the Hecke correspondence $C[g]$ acting on the $L^{2}$ cohomology $H_{(2)}^{*}(X, \mathbf{E})$ was computed by J. Arthur in [Ar1], [Ar3] using the trace formula. In this case the $L^{2}$ cohomology coincides with the "middle" weighted cohomology (see [GHM]), so we obtain an independent computation of this Lefschetz number. In [GKM], it was shown that these two computations agree. Consequently, the present paper completes an independent proof of Arthur's formula.

In the (slightly more general) discrete group setting, the fixed points can be explicitly "counted" using double cosets. This is accomplished in Proposition 8.4 which some geometers may find to be more accessible than the orbital integrals of [GKM] (although they are in fact equivalent). As a consequence, we obtain a Lefschetz formula, Theorem 1.5 (the third main result of this paper), in the discrete group setting. These matters will now be described in more detail.

1.2. Geometric overview. For the purposes of this introduction, a locally symmetric space $X$ is a complete connected Riemannian manifold with finite volume and non-positive curvature, such that every point $p \in X$ has a neighborhood $U_{p}$ with a Cartan symmetry: an isometry $U_{p} \rightarrow U_{p}$ that takes $p$ to itself and induces minus the identity on the tangent space to $X$ at $p$. As for any manifold, we have $X=\Gamma \backslash D$ where $D$ is the simply connected covering space of $X$ and $\Gamma$ is the fundamental group of $X$. Because $X$ has nonpositive curvature, it follows that $D$ is a Riemannian symmetric space of noncompact type, that is, the metric product of a negatively curved symmetric space from Cartan's classification, and a Euclidean factor $\mathbb{R}^{n}$. The discrete group $\Gamma$ acts by Riemannian automorphisms of $D$. We assume that this action is "arithmetic" (see $§ 1.3)$.

Correspondences. We are interested in the automorphisms of $X$. A morphism $f: X \rightarrow Y$ of locally symmetric spaces is a local isometry; i.e. a map $f$ that restricts to an isometry $U_{p} \rightarrow U_{f(p)}$ for appropriate choices of neighborhoods. Topologically, a morphism is a covering map of finite degree. There are finitely many morphisms $X \rightarrow X$. Instead, we consider a correspondence on $X$, i.e. a locally symmetric space $C$ together with two morphisms $c_{1}$ and $c_{2}$ from $C$ to $X$. We can think of $\left(c_{1}, c_{2}\right): C \rightrightarrows X$ as a multi-valued function, whose values at $p \in X$ are the points in the set $c_{2}\left(c_{1}^{-1}(p)\right)$. There is a rich supply of correspondences. They include the Hecke correspondence, see $\$ 1.3$.

Lefschetz numbers. Consider a cohomology theory of $X$, such as the $L^{2}$ cohomology 
$H_{(2)}^{i}(X)$. A correspondence $\left(c_{1}, c_{2}\right): C \rightrightarrows X$ acts on $H_{(2)}^{i}(X)$ by sending a differential form $\omega$ to $C^{*} \omega=\left(c_{1}\right)_{*}\left(c_{2}\right)^{*} \omega$. (The map $\left(c_{1}\right)_{*}$ adds the differential form over the sheets of the finite covering map $c_{1}$.) It is believed that the induced maps $C^{*}: H_{(2)}^{i}(X) \rightarrow H_{(2)}^{i}(X)$ carry deep number theoretic significance. One would like to compute them. Unfortunately, this is too difficult. Even the rank of $H_{(2)}^{i}(X)$ is too hard to compute in most cases. As often happens, however, a more accessible goal is the computation of the Lefschetz number

$$
L(C)=\sum_{i}(-1)^{i} \operatorname{Tr}\left(C^{*}: H_{(2)}^{i}(X) \rightarrow H_{(2)}^{i}(X)\right)
$$

Our goal is to use the Lefschetz fixed point theorem to express the Lefschetz number

$$
L(C)=\sum_{F} L(F)
$$

as a sum over fixed point components $F$ of some local contribution $L(F)$.

Compactifying $X$. The first obstacle is the fact that $X$ is, in general, non-compact. (When $X$ is compact, the Lefschetz formula was described by M. Kuga and J. H. Sampson $[\mathrm{Ku}]$.$) There is no hope for a Lefschetz fixed point formula on a noncompact space. To see$ this, consider the example where $X=\mathbb{C}$ is the complex line with the self map that sends $x \in X$ to $x+1$. The Lefschetz number for ordinary cohomology is 1 . But there are no fixed points, so the right hand side is 0 no matter how $L(F)$ is interpreted. There are similar examples for $L^{2}$ cohomology and locally symmetric spaces $X$.

The solution is to pass to a compactification $\bar{X}$ of $X$. We need a compactification $X \subseteq \bar{X}$ satisfying:

(1) The $L^{2}$ cohomology of $X$ can be expressed locally on $\bar{X}$.

(2) The correspondence $\left(c_{1}, c_{2}\right): C \rightrightarrows X$ extends to a compactified correspondence $\left(c_{1}, c_{2}\right): \bar{C} \rightrightarrows \bar{X}$

(3) The singularities of $\bar{X}$ are simple enough to allow a calculation of $L(F)$.

Remarks on these properties. In (1), "expressed locally" means that the $L^{2}$ cohomology is the cohomology of a complex of sheaves on $\bar{X}$. For (2), we want a functorially constructed compactification $\bar{C}$ of $C$. The map $C^{*}$ on $L^{2}$ cohomology should be induced by a lift of the complex of sheaves to $\bar{C}$. Properties (1) and (2) together will imply that there exists an expression $L(C)=\sum L(F)$ for $L(C)$ as a sum of contributions $L(F)$ over fixed point components $F$ of $\bar{C}$, by applying the fixed point theorem of Grothendieck and Illusie $[\mathrm{GI}]$.

A lot of effort has gone in to constructing various compactifications of $X$. Most of these fail some of the criteria, however. For example, the toroidal compactification [AMRT] for Hermitian $X$ satisfies (3) but neither (1) nor (2). The Borel-Serre compactification [BS] satisfies (2) and (3) but not (1). (It does satisfy (1) in the case of ordinary cohomology rather than $L^{2}$ cohomology. In this case, U. Weselmann, following suggestions of G. Harder, has carried out the Lefschetz computations ([We]).) 
It is likely that (for sufficiently high rank) any compactification satisfying (1) and (2) must be singular. A well known example is the Baily-Borel compactification for Hermitian $X$. This satisfies (1) because of the Zucker conjecture (i.e. the Looijenga [Lo], Saper-Stern [SaS] theorem) which expresses the $L^{2}$ cohomology of $X$ on the Baily-Borel compactification as the intersection cohomology, which is the cohomology of a complex of sheaves (see [GM4]). It satisfies (2) because it is functorial. However, the singularities of the Baily-Borel compactification are as complex as a locally symmetric space only slightly smaller than $X$ and are too complicated to allow a direct computation of $L(F)$.

The first miracle is that there is a compactification satisfying all three properties: the reductive Borel-Serre compactification $\bar{X}$ (defined for all $X$, Hermitian or not). In the Hermitian case, it may be thought of as a (non algebraic) partial resolution of singularities of the Baily-Borel compactification. The reductive Borel-Serre compactification satisfies property (2) because it is functorial with respect to morphisms of locally symmetric spaces. So in the diagram $\left(c_{1}, c_{2}\right): \bar{C} \rightrightarrows \bar{X}$, the space $\bar{C}$ is the reductive Borel-Serre compactification of $C$. It satisfies property (1) because of the existence of weighted cohomology described below, and it satisfies property (3) because its singularities may be explicitly constructed from certain nilmanifolds (see $\$ 1.4$ ).

Weighted cohomology. The Lefschetz fixed point formula of this paper is for the weighted cohomology groups $W^{v} H^{i}(\bar{X}, \mathbf{E})$ where $X$ is any locally symmetric space and $\mathbf{E}$ is a local system over it. These were introduced in $[\mathrm{GHM}]$. The weighted cohomology is the cohomology of a complex of sheaves $\mathbf{W}^{v} \mathbf{C}^{\bullet}(\mathbf{E})$. Therefore it satisfies property (1) mentioned above.

The weighted cohomology groups $W^{v} H^{i}(\bar{X}, \mathbf{E})$ depend on an auxiliary parameter $v$ called a weight profile. When $X$ has finite dimensional $L^{2}$ cohomology, then $W^{v} H^{i}(\bar{X}, \mathbf{E})=H_{(2)}^{i}(X, \mathbf{E})$ provided the weight profile $v$ is chosen to be the "middle weight" ([GHM], $[\mathrm{N}])$, so our formula includes the $L^{2}$ case. Another weight profile gives the ordinary cohomology of $X$. A. Nair $[\mathrm{N}]$ has shown that for any weight profile, the weighted cohomology $W^{v} H^{i}(X, \mathbf{E})$ is equal to J. Franke's "weighted $L^{2}$ cohomology" [F] for a particular weight function. For a leisurely account of the properties of weighted cohomology, see the introduction to [GHM].

The Lefschetz formula. Even on a compact space with mild singularities, the fixed point contribution $L(F)$ is usually too difficult to compute. The second miracle is that on the reductive Borel-Serre compactification $\bar{X}$, each correspondence is hyperbolic. A formula for the contributions $L(F)$ for hyperbolic correspondences was developed in [GM1], [GM2] expressly for the application in this paper to Hecke correspondences. A related result in a different language, which applies to functions (rather than correspondences) was disocvered in $[\mathrm{KS}]$, Prop. 9.6.12.

The rest of the introduction proceeds as follows. The next section enumerates the fixed point components and determines their topology. Section 1.4 describes the local contribution from each fixed point component, and $\$ 1.5$ adds them up to give the Lefschetz number $L(C)$.

1.3. The structure of a correspondence on $\bar{X}$. The theory of correspondences on $\bar{X}$ is very self-referential. The reductive Borel-Serre compactification $\bar{X}$ is a stratified space 
whose strata are themselves locally symmetric spaces. The closure of such a stratum is its reductive Borel-Serre compactification. The fixed point components of a correspondence on $X$ are (almost) locally symmetric. A correspondence restricted to a stratum of $\bar{X}$ is itself a correspondence.

Obtaining $\boldsymbol{X}$ from G. In order to be precise, we need the language of algebraic groups. We use boldface symbols for linear algebraic groups, and Roman symbols for their Lie group of real points, for example, $G=\mathbf{G}(\mathbb{R})$. Throughout this paper we fix a reductive linear algebraic group $\mathbf{G}$ defined over the rational numbers $\mathbb{Q}$. The symmetric space $D$ for $\mathbf{G}$ is defined to be $G / S_{G} K$. Here $K$ is a maximal compact subgroup of $G$ and $\mathbf{S}_{\mathbf{G}}$ is the greatest $\mathbb{Q}$-split torus in the center of $\mathbf{G}$. The group $G$ acts on $D$ by Riemannian automorphisms. Let $X=\Gamma \backslash D=\Gamma \backslash G / S_{G} K$, where $\Gamma \subset \mathbf{G}(\mathbb{Q}) \subset G$ is assumed to be a neat arithmetic subgroup. This is the arithmeticity assumption on the locally symmetric space $X$ of $\S 1.2$. By results of Margulis, in most cases the arithmeticity assumption is automatically satisfied. (The space $D$ may have Euclidean factors because $G$ may have a part of its center that is split over $\mathbb{R}$ but not over $\mathbb{Q}$. After dividing by $\Gamma$, these Euclidean factors will become wound into tori.)

Rational parabolic subgroups $\mathbf{P}$ of $\mathbf{G}$. If $\mathbf{P}$ is a rational parabolic subgroup, let $\mathbf{L}_{\mathbf{P}}$ be its Levi quotient; let $\nu_{\mathbf{P}}: \mathbf{P} \rightarrow \mathbf{L}_{\mathbf{P}}$ be the projection; let $\mathscr{U}_{\mathbf{P}}$ be the unipotent radical; let $\mathbf{S}_{\mathbf{P}} \subseteq \mathbf{L}_{\mathbf{P}}$ be the maximal $\mathbb{Q}$-split torus in the center of $\mathbf{L}_{\mathbf{P}}$; and let $\Delta_{P}$ be the set of simple roots occurring in $\mathfrak{N}_{P}=\operatorname{Lie}\left(\mathscr{U}_{P}\right)$. Let $K_{P}=K \cap L_{P}$ be the maximal compact subgroup of $L_{P}$ which corresponds to $K$; set $\Gamma_{P}=\Gamma \cap P$; and $\Gamma_{L}=v_{P}\left(\Gamma_{P}\right)$.

The reductive Borel-Serre compactification $\bar{X}(\$ 2.10)$. The strata of $\bar{X}$ are indexed by $\Gamma$-conjugacy classes of rational parabolic subgroups $\mathbf{P}$ of $\mathbf{G}$. The stratum $X_{P} \subseteq \bar{X}$ corresponding to the conjugacy class containing a parabolic $\mathbf{P}$ is the locally symmetric space $\Gamma_{L} \backslash D_{P}$, where $D_{P}=L_{P} / S_{P} K_{P}$ is the symmetric space of the Levi factor $\mathbf{L}_{\mathbf{P}}$. If $\mathbf{P} \subseteq \mathbf{Q}$ then the stratum $X_{P}$ is contained in the closure $\bar{X}_{Q}$ of $X_{Q}$ (which is the reductive Borel-Serre compactification of $X_{Q}$ ).

Hecke correspondences. Let $g \in \mathbf{G}(\mathbb{Q})$. Let $\Gamma^{\prime} \subset \Gamma \cap g^{-1} \Gamma g$ be a subgroup of finite index. This data determines a correspondence $\left(c_{1}, c_{2}\right): C\left[g, \Gamma^{\prime}\right] \rightrightarrows X$ as follows. Let $C\left[g, \Gamma^{\prime}\right]=\Gamma^{\prime} \backslash D$. The mapping $c_{1}$ is obtained by factoring the projection $d_{1}: D \rightarrow \Gamma \backslash D=X$ through $C\left[g, \Gamma^{\prime}\right]$ which may be done since $\Gamma^{\prime} \subset \Gamma$. The mapping $c_{2}$ is obtained by factoring the projection $d_{2}: D \rightarrow \Gamma \backslash D$ through $C\left[g, \Gamma^{\prime}\right]$, where $d_{2}(x)=d_{1}(g x)$. Such a factoring exists because $\Gamma^{\prime} \subset g^{-1} \Gamma g$.

It is a fact (Proposition 6.9) that every correspondence may be obtained in this way. For the maximal choice $\Gamma^{\prime}=\Gamma \cap g^{-1} \Gamma g$, the resulting correspondence is called a Hecke correspondence and is denoted $C[g] \rightrightarrows X$. Up to isomorphism, this correspondence depends only on the double coset $\Gamma g \Gamma \in \Gamma \backslash \mathbf{G}(\mathbb{Q}) / \Gamma$ (cf. Lemma 6.6).

The correspondence $C\left[g, \Gamma^{\prime}\right] \rightrightarrows X$ is a covering of the correspondence $C[g] \rightrightarrows X$ of degree $d=\left[\Gamma: \Gamma^{\prime}\right]$. The action of $C\left[g, \Gamma^{\prime}\right]$ on weighted cohomology is simply $d$ times the action of $C[g]$. So, without loss of generality, we may concentrate on computing the Lefschetz number of the Hecke correspondence $C[g]$ for a fixed double coset $\Gamma g \Gamma \in \Gamma \backslash \mathbf{G}(\mathbb{Q}) / \Gamma$.

The correspondence on a stratum of $\bar{X}$. Each Hecke correspondence $C[g] \rightrightarrows X$ has a 
unique continuous extension to the reductive Borel-Serre compactification $\bar{C}[g] \rightrightarrows \bar{X}$. Every boundary stratum $C_{Q}$ of $\bar{C}[g]$ will be a correspondence taking a boundary stratum of $\bar{X}$ to another one. Since we are interested in fixed points, we want to classify those $C_{Q}$ which take a stratum $X_{P} \subseteq \bar{X}$ to itself. There is one of these for every double coset $\Gamma_{P} g_{i} \Gamma_{P}$ in the intersection $P \cap \Gamma g \Gamma$ (Proposition 7.3). It is isomorphic to a correspondence of the form $C\left[\bar{g}_{i}, \Gamma_{L}^{\prime}\right]$ as described above, but with $G$ replaced by $L_{P}$. (Here $\bar{g}_{i}=v_{P}\left(g_{i}\right) \in L_{P}$ and $\left.\Gamma_{L}^{\prime}=v_{P}\left(\Gamma_{P} \cap g_{i}^{-1} \Gamma g_{i}\right) \subset L_{P}.\right)$

Fixed point components. The fixed point set (sometimes called the coincidence set) of a correspondence $\left(c_{1}, c_{2}\right): C \rightrightarrows X$ is by definition the set of points in $C$ on which the two maps $c_{1}$ and $c_{2}$ agree.

The fixed point set of the correspondence $C\left[g, \Gamma^{\prime}\right] \rightrightarrows X$ (before compactification) breaks up into constituent pieces $F(e)$ indexed by $\Gamma$ conjugacy classes of elliptic (modulo $S_{G}$ ) elements $e \in \Gamma g \Gamma(\$ 8.2)$. The piece $F(e)$ corresponding to the conjugacy class containing $e$ is the space $\Gamma_{e}^{\prime} \backslash G_{e} / K_{e}^{\prime}$, where $G_{e}$ is the centralizer of $e$ in $G, \Gamma_{e}^{\prime}=\Gamma^{\prime} \cap G_{e}$, and $K_{e}^{\prime}=G_{e} \cap\left(z\left(S_{G} K\right) z^{-1}\right)$ where $z A_{G} K \in D=G / A_{G} K$ is a fixed point of the action of $e$ on $D$. (Such a point exists since $e$ is elliptic (§8.4).) The constituent $F(e)$ is a finite union of spaces, each of which is almost a locally symmetric space for the group $G_{e}$. (It may have infinite volume because it may have Euclidean factors that are not wound into tori.)

Applying this result to the boundary stratum $X_{P}$, in conjunction with the calculation $(\S 1.3)$ of the part of the correspondence $\bar{C}[g]$ which preserves $X_{P}$, we get a group theoretic enumeration of all the fixed points of $\bar{C}[g]$ which lie over points in $X_{P}$ : For each choice of a double coset $\Gamma_{P} g_{i} \Gamma_{P} \subset \Gamma g \Gamma \cap P$, and for each conjugacy class of elliptic (modulo $S_{P}$ ) elements $e$ in $\Gamma_{L} \bar{g}_{i} \Gamma_{L}$, there is a fixed point constituent $F_{P}(e)$. (It is a smooth submanifold of the stratum $C_{Q}$ of $\bar{C}[g]$ which is determined by the double coset $\Gamma_{P} g_{i} \Gamma_{P}$ as in $\S 1.3$.) Summing over $\Gamma$ conjugacy classes of rational parabolics $P$ gives the complete enumeration of fixed points of $\bar{C}[g]$.

The topology of the fixed point set. There are finitely many constituents $F_{P}(e)$ of the fixed point set and they are disjoint. Unfortunately however they may not be topologically isolated from each other. If $X_{P^{\prime}} \subseteq \bar{X}_{P}$, then the closure of $F_{P}(e)$ can contain points in some $F_{P^{\prime}}\left(e^{\prime}\right)$. So a single connected component of the fixed point set may have a very complicated structure. This phenomenon is the main source of technical difficulty of this paper. (The only real limit we have found on the possible complexity of a connected component of the fixed point set is Proposition 10.4.) We get around this problem by composing the correspondence with a mapping, very close to the identity, which shrinks a neighborhood of the singularity set $\bar{X}-X$ into the singularity set, and which does something similar on the closure of each stratum of $\bar{X}$. This has the effect of "truncating" each connected component of the fixed point set into pieces each of which is contained in a single stratum of $\bar{X}$ (a process which may be considered as a sort of topological analog to Arthur's truncation procedure). The Lefschetz number of this "modified" Hecke correspondence is equal to that of the original one. We prove that the modified Hecke correspondence is hyperbolic. The resulting formula (Theorem 1.5) would be the same if no truncation were used, however the proof would be more technical.

1.4. Calculating the local contribution $\boldsymbol{L}(\boldsymbol{F})$. Let $E$ be a finite dimensional representation of $\mathbf{G}$, and let $\mathbf{E}$ be the associated local system over $X$. Denote by $\mathbf{P}_{\mathbf{0}}$ a fixed min- 
imal ("standard") rational parabolic subgroup of $G$ and by $\mathbf{S}_{\mathbf{0}}$ a maximal $\mathbb{Q}$-split torus in the center of its Levi factor. Then $\mathbf{S}_{\mathbf{G}} \subseteq \mathbf{S}_{\mathbf{0}}$. A weight profile $v \in \chi_{\mathbb{Q}}^{*}\left(\mathbf{S}_{\mathbf{0}}\right)(\S 12.2)$ is a (quasi-) character of $\mathbf{S}_{\mathbf{0}}$ whose restriction to $\mathbf{S}_{\mathbf{G}}$ coincides with the character by which $\mathbf{S}_{\mathbf{G}}$ acts on $E$. The Hecke correspondence $\bar{C}[g] \rightrightarrows \bar{X}$ has a canonical lift (\$13.1) to the weighted cohomology sheaf $\mathbf{W}^{v} \mathbf{C}^{\bullet}(\mathbf{E})$, so it induces a homomorphism on weighted cohomology whose Lefschetz number

$$
L(C[g])=\sum_{i \geqq 0}(-1)^{i} \operatorname{Tr}\left(C[g] ; W^{v} H^{i}(\bar{X} ; \mathbf{E})\right)
$$

is what we want to compute.

Hyperbolicity of the correspondence. Let us assume for the moment that the fixed point set is topologically the disjoint union of the constituent pieces $F_{P}(e)$. This is not always the case, but the formula we obtain is nevertheless always valid, as explained in $\S 1.3$.

We focus on a single stratum $X_{P}$ which is preserved by the correspondence, and on a single stratum of the correspondence $\bar{C}$ corresponding to a single double coset $\Gamma_{P} g_{i} \Gamma_{P} \subset P \cap \Gamma g \Gamma$. Within this stratum, we focus on a single constituent $F_{P}(e)$ of the fixed point set. Each stratum $X_{Q}$ which contains $X_{P}$ in its closure correspondence to a rational parabolic subgroup $\mathbf{Q}$ containing $\mathbf{P}$, and therefore to a unique subset $I \subset \Delta_{P}$. The empty subset corresponds to $X_{P}$ itself and the largest subset $\Delta_{P} \subseteq \Delta_{P}$ corresponds to $X$. Let $a_{e}$ be the projection of $e$ to the identity component $A_{P}$ of $\mathbf{S}_{\mathbf{P}}(\mathbb{R})$. The elements of $\Delta_{P}$ are rational characters of $\mathbf{S}_{\mathbf{P}}$ so we may define

$$
\Delta_{P}^{+}(e)=\left\{\alpha \in \Delta_{P} \mid \alpha\left(a_{e}\right)<1\right\} .
$$

Let $X_{Q}$ be the stratum containing $X_{P}$ which corresponds to the subset $\Delta_{P}^{+}(e) \cong \Delta_{P}$. The correspondence $C[g]$ is hyperbolic near $F_{P}(e)$ (11.7), with "expanding" (or "unstable") set $X_{Q}$ (Theorem 11.9, §13.10). In other words, near $F_{P}(e)$ the Hecke correspondence is "expanding" in those directions normal to $X_{P}$ which point into $X_{Q}$.

Let $F^{\prime}=c_{1}\left(F_{P}(e)\right)=c_{2}\left(F_{P}(e)\right) \subset X_{P}$ and let $L_{e}$ be the centralizer of $e$ in $L_{P}$. There are diffeomorphisms (Proposition 8.4),

$$
F_{P}(e) \cong \Gamma_{e}^{\prime} \backslash L_{e} / K_{e}^{\prime} \quad \text { and } \quad F^{\prime} \cong \Gamma_{e} \backslash L_{e} / K_{e}^{\prime}
$$

where $K_{e}^{\prime}=L_{e} \cap z\left(K_{P} A_{P}\right) z^{-1}$ (for appropriately chosen $\left.z \in L_{P}\right), \quad \Gamma_{e}=L_{e} \cap \Gamma_{L}$, and $\Gamma_{e}^{\prime}=L_{e} \cap \Gamma_{L}^{\prime}$. The projection $F_{P}(e) \rightarrow F^{\prime}$ is a covering of degree $d_{e}=\left[\Gamma_{e}: \Gamma_{e}^{\prime}\right]$ (cf. §8.6).

It follows from the Lefschetz fixed point theorem of [GM2] that the local contribution is given by

$$
L\left(F_{P}(e)\right)=\chi_{c}\left(F^{\prime}\right) \sum_{i \geqq 0}(-1)^{i} \operatorname{Tr}\left(C[g]^{*}: H_{x}^{i}\left(\mathbf{A}^{\bullet}\right) \rightarrow H_{x}^{i}\left(\mathbf{A}^{\bullet}\right)\right)
$$

(see Theorem 13.2). Here $\mathbf{A}^{\bullet}=h^{!} j^{*} \mathbf{W}^{v} \mathbf{C}^{\bullet}(E)$ where $h$ is the inclusion $F^{\prime} \hookrightarrow X_{P} \hookrightarrow \bar{X}_{Q}$ and $j$ is the inclusion $\bar{X}_{Q} \hookrightarrow \bar{X} ; H_{x}^{i}\left(\mathbf{A}^{\bullet}\right)$ denotes its stalk cohomology at $x \in F^{\prime}$; and $\chi_{c}$ denotes 
the Euler characteristic with compact supports. See the introduction to [GM2] for a geometric account of hyperbolic correspondences.

The stalk cohomology. Let $c$ denote the codimension of $F^{\prime}$ in $X_{P}$ and let $\mathcal{O}$ be the top exterior power of the normal bundle of $F^{\prime}$ in $X_{P}$. Let $r$ denote the index $\left[\Gamma_{P} \cap \mathscr{U}_{P}: \Gamma_{P}^{\prime} \cap \mathscr{U}_{P}\right]$ where $\Gamma_{P}^{\prime}=\Gamma_{P} \cap g_{i}^{-1} \Gamma_{P} g_{i}$.

The stalk cohomology of the sheaf $\mathbf{A}^{\bullet}$ at a fixed point $x \in F^{\prime} \subset X_{P}$ is given by (13.10.4) and Proposition 12.8:

$$
H_{x}^{\bullet}\left(\mathbf{A}^{\bullet}\right) \cong \bigoplus_{\substack{w \in W_{P}^{1} \\ I_{v}(w)=\Delta_{P}^{+}(e)}} V_{w\left(\lambda_{B}+\rho_{B}\right)-\rho_{B}}^{L}\left[-\ell(w)-\left|\Delta_{P}^{+}(e)\right|-c\right] \otimes \mathcal{O}_{x}
$$

The Hecke correspondence $\bar{C}[g]$ acts on the first factor by $r d_{e}$ times the action of $e^{-1}$ and it acts on the second factor by $(-1)^{c}$, cf. (13.10.5). We now describe the other symbols in this formula.

Let $\mathbf{T}$ be a maximal torus (over $\mathbb{C}$ ) in $\mathbf{G}$, and let $\mathbf{B}$ be a Borel subgroup (over $\mathbb{C}$ ) of $\mathbf{G}$ containing $\mathbf{T}$. These may be chosen as in $\$ 12.7$ so that $\mathbf{S}_{\mathbf{0}}(\mathbb{C}) \subset \mathbf{T}(\mathbb{C})$ and so that $\mathbf{B} \subset \mathbf{P}_{\mathbf{0}}$. Let $W_{G}=W(\mathbf{G}(\mathbb{C}), \mathbf{T}(\mathbb{C}))$ denote the Weyl group of $\mathbf{G}$. The choice of $\mathbf{B}$ determines positive roots $\Phi_{G}^{+}=\Phi^{+}(\mathbf{G}(\mathbb{C}), \mathbf{T}(\mathbb{C}))$, and a length function $\ell$ on $W_{G}$. Let $W_{P}^{1} \subset W_{G}$ denote the set of Kostant representatives: the unique elements of minimal length from each of the cosets $W_{P} x \in W_{P} \backslash W_{G}$, where $W_{P}=W\left(\mathbf{L}_{\mathbf{P}}(\mathbb{C}), \mathbf{T}(\mathbb{C})\right),(\S 12.7)$. The sum in (1.4.2) is over those $v \in W_{P}^{1}$ such that the set

$$
I_{v}(w)=\left\{\alpha \in \Delta_{P} \mid\left\langle w\left(\lambda_{B}+\rho_{B}\right)-\rho_{B}-v, t_{\alpha}\right\rangle<0\right\}
$$

coincides with the set $\Delta_{P}^{+}(e)$ defined above (after conjugating $\mathbf{P}$ so as to contain $\left.\mathbf{B}\right)$. Here, as in $\$ 12.7, \lambda_{B}$ denotes the highest weight of the representation $E$, and $\left\{t_{\alpha}\right\}$ form the basis of the cocharacter group $\chi_{*}^{\mathbb{Q}}\left(\mathbf{S}_{\mathbf{P}} / \mathbf{S}_{\mathbf{G}}\right)$ which is dual to the basis $\Delta_{P}$ of the simple roots. Also, $\rho_{B}$ denotes the half-sum of the positive roots $\Phi_{G}^{+}$. The product $\left\langle v\left(\lambda_{B}+\rho_{B}\right)-\rho_{B}-v, t_{\alpha}\right\rangle$ makes sense: the restriction $\left(v\left(\lambda_{B}+\rho_{B}\right)-\rho_{B}-v\right) \mid \mathbf{S}_{\mathbf{P}}$ is trivial on $\mathbf{S}_{\mathbf{G}}$ and hence defines an element of $\chi^{*}\left(\mathbf{S}_{\mathbf{P}}^{\prime}\right) \otimes \mathbb{Q}$ which can then be paired with $t_{\alpha}$. For any B-dominant weight $\beta$, the symbol $V_{\beta}^{L}$ denotes the irreducible $\mathbf{L}_{\mathbf{P}}$-module with highest weight $\beta \in \chi^{*}(\mathbf{T}(\mathbb{C}))$ and $V_{\beta}^{L}[-m]$ means that the module $V_{\beta}^{L}$ is placed in degree $m$.

The geometry behind this formula is roughly this: Consider the intersection of a small neighborhood of $x$ in $\bar{X}$ with the largest stratum $X$. This intersection will deformation retract to the nilmanifold $\left(\Gamma \cap \mathscr{U}_{P}\right) \backslash \mathscr{U}_{P}$. The cohomology of this intersection with coefficients in $\mathbf{E}$ coincides with the $\mathfrak{N}_{P}$ cohomology by Van Est's theorem, which is computed by Kostant's theorem to be $\bigoplus_{w \in W_{P}^{1}} V_{w\left(\lambda_{B}+\rho_{B}\right)-\rho_{B}}^{L}[-\ell(w)]$. The cut-off $\left(I_{v}(w)=\Delta_{P}^{+}(e)\right)$ and the degree shift (by $\ell(w)+\left|\Delta_{P}^{+}(e)\right|$ ) come from the computation of $h^{!} j^{*} \mathbf{W}^{v} \mathbf{C}^{\bullet}(E)$ in $\S 12$. The integer $r$ is the ramification index: the degree of the mapping $c_{1}$ when it is restricted to this nilmanifold (\$13.9).

By adding the contributions $L\left(F_{P}(e)\right)$ over all the fixed point constituents $F_{P}(e)$ we arrive at the final result in this paper. It is proven in $\S 14$. 
1.5. Theorem. Let $g \in \mathbf{G}(\mathbb{Q})$. Let $\bar{C}[g] \rightrightarrows \bar{X}$ be the resulting Hecke correspondence. Fix a weight profile $v \in \chi_{\mathbb{Q}}^{*}\left(\mathbf{S}_{\mathbf{0}}\right)$. The Lefschetz number $L(C[g])(1.4 .1)$ is given by

$$
\sum_{[\mathbf{P}]} \sum_{i} \sum_{\{e\}} r \chi_{c}\left(\Gamma_{e}^{\prime} \backslash L_{e} / K_{e}^{\prime}\right)(-1)^{\left|\Delta_{P}^{+}(e)\right|} \sum_{\substack{w \in W_{P}^{1} \\ I_{v}(w)=\Delta_{P}^{+}(e)}}(-1)^{\ell(w)} \operatorname{Tr}\left(e^{-1} ; V_{w\left(\lambda_{B}+\rho_{B}\right)-\rho_{B}}^{L}\right) .
$$

The first sum is over a choice of representative $\mathbf{P}$, one from each $\Gamma$-conjugacy class of rational parabolic subgroups of $\mathbf{G}$. For such a $\mathbf{P}$, set $\Gamma g \Gamma \cap \mathbf{P}(\mathbb{Q})=\coprod_{i} \Gamma_{P} g_{i} \Gamma_{P}$ (where $\Gamma_{P}=\Gamma \cap \mathbf{P}(\mathbb{Q})$ and where $\left.g_{i} \in \mathbf{P}(\mathbb{Q})\right)$. The second sum is over these finitely many double cosets. Set $\bar{g}_{i}=v_{P}\left(g_{i}\right) \in L_{P}$ and $\Gamma_{L}=v_{P}\left(\Gamma_{P}\right)$. The third sum is over a choice of representatives $e$, one from each $\Gamma_{L}$-conjugacy class of elliptic (modulo $S_{P}$ ) elements $e \in \Gamma_{L} \bar{g}_{i} \Gamma_{L}$. The rest of the notations are explained above.

There are various ways to rewrite the Lefschetz formula; see $\S 14.4, \S 15.8$ and $\S 15.9$.

1.6. Adelic formulation. One of the main goals of the series of papers [GM1], [GM2], [GHM], [GKM], and the present paper is Theorem 7.14.B (p. 535) of [GKM], an expression for the Lefschetz number $L(C[g])$ in the adelic setting. If the weight profile $v$ is the "middle" weight (and if the rank of $\mathbf{G}$ equals the rank of $\mathbf{K}$ ) then the weighted cohomology coincides with the $L^{2}$ cohomology, and this formula coincides with Arthur's formula [Ar1] (Theorem 6.1). If the weight profile $v=-\infty$ then the weighted cohomology coincides with the ordinary "full cohomology" $H^{*}(X, E)$ and this formula coincides with Franke's formula $[\mathrm{F}]($ thm. 21, p. 273). The paper $[\mathrm{GKM}]$ uses the above Theorem 1.5 as its starting point (see the remarks following Theorem 13.6), then modifies it using three main steps.

(1) The quantity $r \chi_{c}\left(\Gamma_{e}^{\prime} \backslash L_{e} / K_{e}^{\prime}\right)$ which appears in Theorem 1.5, and the sum $\sum_{i}$ over double cosets $\Gamma_{P} g_{i} \Gamma_{P} \subset \Gamma g \Gamma \cap P$ (which precedes it) are replaced by an orbital integral.

(2) If $L_{P} / A_{P}$ does not contain a compact maximal torus, then the stratum $C_{P}$ makes no contribution to Arthur's formula or to Franke's formula. The same holds for the general formula in Theorem 7.14.B of [GKM]. However fixed points in such a stratum may make a nonzero contribution to the formula in Theorem 1.5 above. In [GKM], §7.14 the method of descent is used to re-attribute such a contribution to smaller strata $C_{Q}$ for which $L_{Q} / A_{Q}$ does admit a compact maximal torus. See also $§ 15.8$ of this paper.

(3) Theorem 1.5 above involves a sum over parabolic subgroups, while Theorem 7.14.B of $[\mathrm{GKM}]$ involves a sum over Levi subgroups. This is achieved in $[\mathrm{GKM}]$ (p. 529) by grouping together the contributions from those parabolic subgroups with a given Levi factor. (This has the remarkable effect of grouping together fixed points with different contracting-expanding behavior.) In $[\mathrm{GKM}]$ it is shown that the resulting contribution from a single Levi subgroup may be interpreted in terms of the (Harish-Chandra) character of a certain admissible representation. In the case of the middle weight, this fact gives rise to a combinatorial formula for the stable discrete series characters, which is the second main result of $[\mathrm{GKM}]$. (Although this discrete series character formula was discovered by comparing Arthur's formula to Theorem 1.5, the statement and proof of the character formula in $[\mathrm{GKM}]$ is independent of the part of the paper dealing with Lefschetz numbers.) 
1.7. Related literature. Besides the articles listed above, and an extensive literature on the co-compact case, we mention several other closely related papers. The Lefschetz formula in the rank one case was studied by Moscovici [Mo] and Barbasch-Moscovici $[\mathrm{BaM}]$, also by Bewersdorff $[\mathrm{Be}]$ and Rapoport $[\mathrm{R}]$. In [St], M. Stern gave a general Lefschetz formula for Hecke correspondences. We do not easily see how to compare his formula with ours. In [Sh] S. Shokranian, following the outline in $[\mathrm{GKM}]$, describes a formula for the Lefschetz numbers of Hecke operators on twisted groups. We wish to draw attention to Langlands' article [L1], in which the expanding and contracting nature of the fixed points on the boundary was first isolated (see especially Proposition 7.12, p. 485).

1.8. Acknowledgments. We would like to thank J. Arthur, W. Casselman and R. Langlands for encouraging us to work on this question. We would especially like to thank R. Kottwitz for patiently explaining Arthur's formula to us and for helping to interpret our early results in this direction. Some of the results in this paper appeared earlier in the adelic setting in our joint paper $[\mathrm{GKM}]$ with R. Kottwitz. We have profited from useful conversations with A. Nair, A. Borel, W. Casselman, P. Deligne, G. Harder, E. Looijenga, S. Morel, A. Nicas, M. Rapoport, L. Saper, J. Steenbrink, M. Stern, and S. Zucker. The first author is grateful to the Institute for Advanced Study for its support while much of this paper was written. This research was begun and partially completed when the authors were at Northeastern University and the Massachusetts Institute of Technology, respectively. We are also grateful to the following institutions for their hospitality and support during various phases of this project: the Centre de Recherches Mathématiques at the Université de Montréal, the Max Planck Institut für Mathematik in Bonn, the Department of Mathematics at the University of Chicago, the Universita di Roma la Sapienza. This research was partially supported by the National Science Foundation under grants number DMS-8802638, DMS-9001941, DMS-9303550, DMS-9626616, DMS-9900324, DMS-0139986(Goresky) and DMS-8803083, DMS-9106522(MacPherson).

\subsection{List of symbols.}

§2. $\quad$ 2.1: $\mathbf{G}, \mathbf{S}_{\mathbf{G}}, A_{G},{ }^{\mathbf{0}} \mathbf{G}, K, D, \mathbf{G}^{(\mathbf{1})}, K^{(1)}, A_{0}, T_{g}, K(x), \psi_{x}, K^{\prime}$, elliptic, $\theta, \Gamma, X, \tau$

2.2: $\mathbf{P}, \mathscr{U}_{P}, R_{d} P, L_{P}, v_{P}, M_{P}, \mathbf{S}_{\mathbf{P}}, A_{P}, \Gamma_{P}, K_{P}, \mathbf{S}_{\mathbf{P}}^{\prime}, A_{P}^{\prime}, i_{x_{0}}$, Langlands' decomposition, $a_{g}$, geodesic action, torus factor

2.3: $\quad \mathbf{P}_{\mathbf{0}}, \mathbf{S}_{\mathbf{0}}, \Phi, \mathfrak{N}_{0}, \Delta, \mathbf{P}_{0}(I), \chi_{Q}\left(\mathbf{S}_{\mathbf{P}}^{\prime}\right)$

2.4: $\overline{\mathbf{P}}, i\left(\Delta_{Q}\right)$, complementary decomposition, orthogonal decomposition

2.5: boundary component, boundary stratum, $e_{P}, Y_{P}, D_{P}, X_{P}, \mu, F_{P}$, canonical cross section

2.6: $\mathscr{M}_{x}$

2.9: $\quad \bar{A}_{P}^{\prime}, A_{P}^{\prime}(>1), \bar{A}_{P}^{\prime}(\geqq 1), \tilde{D}, D(P), \tilde{X}$

2.10: $\pi_{P}$, geodesic projection, $\bar{D}, \mu, D[P], \bar{X}, \tau, X(P), X[P]$

§3. $3.1: \quad \alpha, \beta, \Gamma$-parabolic

3.2: root function, $f_{\alpha}^{P}, \pi_{P}$ 
§4. $\quad$ 4.1: $\quad \mathscr{P}_{1}, \mathbf{b}$, parameter, $\mathscr{B}$, tiling, $D^{P}, \partial^{P} D^{0}, D_{Q}^{0}$

4.2: $\quad T\left(\bar{D}_{Q}\right), \partial T\left(\bar{D}_{Q}\right), r_{\alpha}^{Q}$, partial distance function

4.5: $\quad X^{P}, T\left(\bar{X}_{P}\right), \partial T\left(\bar{X}_{P}\right)$

4.6: $\quad R$, retraction, $W$, exhaustion, $R_{Q}, W_{Q}$

§5. $\quad$ 5.2: $\quad D\{Q\}, \rho, \operatorname{Sh}(Q, t), S h_{\mathbf{Q}}(t)$

5.4: $\operatorname{Sh}(\mathbf{t})$

§6. $\quad 6.2$ : morphism, $\operatorname{Mor}\left(X^{\prime}, X\right)$

6.3: $\tilde{f}, \bar{f}$

6.5: correspondence, $\Gamma[g], C[g]$

6.11: narrow

§7. $\quad$ 7.1: parabolic correspondence, $\Gamma_{P}[y]$, modeled

7.3: $\Xi$

§8. 8.2: fixed point, characteristic element, $e, F_{P}(e)$, elliptic, $L_{e}$

§9. 9.1: $\Delta_{P}^{+}, \Delta_{P}^{-}, \Delta_{P}^{0}$

9.2: neutral, $\mathbf{P} \prec \mathbf{Q}$

9.4: $\quad P^{\dagger}$

§11. 11.4: $E, d_{E}$

11.7: hyperbolic

§12. 12.1: $t_{\alpha}, \chi_{\mathbb{Q}}^{*}\left(\mathbf{S}_{\mathbf{P}}\right)_{\left\lceil v_{P}, J\right]}, \chi_{\mathbb{Q}}^{*}\left(\mathbf{S}_{\mathbf{P}}\right)_{\geqq v_{P}(J)}$

12.2: $\mathbf{E}, v$, weight profile, $\mathbf{W}^{v} \mathbf{C}^{\bullet}(\mathbf{E})$

12.3: $\mathcal{O}_{X / Y}$

12.6: $N_{y}, \mathscr{L}_{y}, \delta, \triangleright^{s-1}, \triangleright_{J}$

12.7: $\Phi_{G}^{+}, \Phi_{L}^{+}, \rho_{B}, W_{G}, W_{P}, W_{P}^{1}, V_{\beta}^{L}, \lambda_{B}, I_{v}(w)$

§13. 13.1: $\mathbf{A}^{\bullet}$

13.2: $\chi_{c}$

13.5: $r$

13.7: $N_{P}$

13.9: $\mathbf{C}^{\bullet}\left(N_{P}, \mathbf{E}\right), C^{\bullet}\left(\Re_{P}, E\right), \Omega_{\text {inv }}\left(\mathscr{U}_{P}, E\right)$ 


\section{Notation and terminology}

2.1. Locally symmetric spaces. Linear algebraic groups will be represented by boldface symbols (e.g., $\mathbf{G}, \mathbf{S}$ ) and their real points will be in Roman type (e.g., $G=\mathbf{G}(\mathbb{R})$, $S=\mathbf{S}(\mathbb{R}))$. Throughout this paper we fix a connected reductive linear algebraic group $\mathbf{G}$ defined over $\mathbb{Q}$. Denote by $\mathbf{S}_{\mathbf{G}}$ the greatest $\mathbb{Q}$-split torus in the center of $G$, and let $A_{G}=\mathbf{S}_{\mathbf{G}}(\mathbb{R})^{0}$ denote the identity component of the group of real points of $\mathbf{S}_{\mathbf{G}}$. Following [BS], §1.1 let

$$
{ }^{\mathbf{0}} \mathbf{G}=\bigcap_{\chi} \operatorname{ker}\left(\chi^{2}\right)
$$

be the intersection of the kernels of the squares of all the rationally defined characters $\chi: \mathbf{G} \rightarrow \mathbf{G L}_{\mathbf{1}}$. Then ${ }^{0} G$ is normal in $G$; it contains every compact subgroup and every arithmetic subgroup of $G$, and $G=A_{G} \times{ }^{0} G$. Let $K \subset \mathbf{G}(\mathbb{R})$ be a maximal compact subgroup and define $D=G / K A_{G}$. We refer to $D$ as the "symmetric space" associated to $\mathbf{G}$. The derived group $\mathbf{G}^{(\mathbf{1})}$ is semisimple and $K^{(1)}=G^{(1)} \cap K$ is a maximal compact subgroup. The space $D$ is diffeomorphic to the Cartesian product of the Riemannian symmetric space $D^{(1)}=G^{(1)} / K^{(1)}$ with $A_{0} / A_{G}$ where $A_{0}$ is the identity component of the greatest $\mathbb{R}$-split torus in the center of $G$. Both $\mathbf{G}(\mathbb{R})$ and ${ }^{0} G$ act transitively on $D$, an action which we usually denote by $(g, x) \mapsto g x$ or $g . x$ but occasionally it will be necessary to refer to this action as a mapping, in which case we write

$$
T_{g}: D \rightarrow D
$$

for $g \in G$. (For most geometric questions involving the symmetric space $D$, one could replace $G$ by ${ }^{0} G$, however there are Hecke correspondences for $G$ which do not necessarily come from ${ }^{0} G$.) For each $x \in D$ the stabilizer $K(x)$ of $x$ in ${ }^{0} G$ is a maximal compact subgroup of ${ }^{0} G$ so we obtain a $G$-equivariant diffeomorphism

$$
\psi_{x}: G / A_{G} K(x) \rightarrow D .
$$

The choice of $K \subset G$ corresponds to a "standard" basepoint $x_{0} \in D$. We write $K=K\left(x_{0}\right)$ and $K^{\prime}=A_{G} K\left(x_{0}\right)$. An element $x \in G$ is elliptic $\bmod A_{G}$ (often shortened to "elliptic") if it is $\mathbf{G}(\mathbb{R})$-conjugate to an element of $K^{\prime}$. There is a unique "algebraic" Cartan involution $\theta=\theta_{x_{0}}: G \rightarrow G$ whose fixed point set is $K$. If $x_{1} \in D$ is another basepoint with $x_{1}=g x_{0}$ then the Cartan involution for the new basepoint is given by

$$
\theta_{x_{1}}(y)=g \theta_{x_{0}}\left(g^{-1} y g\right) g^{-1}
$$

and the composition $\psi_{x_{1}}^{-1} \psi_{x_{0}}: G / A_{G} K\left(x_{0}\right) \rightarrow G / A_{G} K\left(x_{1}\right)$ is given by

$$
y A_{G} K\left(x_{0}\right) \mapsto y g^{-1} A_{G} K\left(x_{1}\right) .
$$

Let $\mathfrak{g}=\mathfrak{f} \oplus \mathfrak{p}$ be the \pm 1 eigenspace decomposition of $\theta$ in $\operatorname{Lie}(G)$. The Cartan involution $\theta$ preserves ${ }^{0} G$ and determines a decomposition of its Lie algebra, ${ }^{0} \mathfrak{g}=\mathfrak{f} \oplus{ }^{0} \mathfrak{p}$. Then ${ }^{0} \mathfrak{p}$ may be canonically identified with the tangent space $T_{x_{0}} D$. Any choice of $K$-invariant inner product on ${ }^{0} \mathfrak{p}$ induces a $G$-invariant Riemannian metric on $X$.

Throughout this paper we also fix an arithmetic subgroup $\Gamma \subset \mathbf{G}(\mathbb{Q})$ and denote by $\tau: D \rightarrow X=\Gamma \backslash D$ the projection to the locally symmetric space $X$. 
2.2. Parabolic subgroups. Fix a rationally defined parabolic subgroup $\mathbf{P} \subset \mathbf{G}$. We have the following groups:

(1) $\mathscr{U}_{P}=$ the unipotent radical of $\mathbf{P} ; \mathfrak{N}_{P}=\operatorname{Lie}\left(\mathscr{U}_{P}\right)$ its Lie algebra.

(2) $\mathbf{R}_{d} \mathbf{P}=$ the $\mathbb{Q}$ split radical of $\mathbf{P}$.

(3) $\mathbf{L}_{\mathbf{P}}=$ the Levi quotient; $v_{P}: \mathbf{P} \rightarrow \mathbf{L}_{\mathbf{P}}$ the projection.

(4) $\mathbf{M}_{\mathbf{P}}={ }^{0} \mathbf{L}_{\mathbf{P}}=\bigcap_{\chi} \operatorname{ker}\left(\chi^{2}\right)$.

(5) $\mathbf{S}_{\mathbf{P}}=\mathbf{R}_{d} \mathbf{P} / \mathscr{U}_{\mathbf{P}}$

(6) $A_{P}=\mathbf{S}_{\mathbf{P}}(\mathbb{R})^{0}$ the identity component of the set of real points.

(7) $\Gamma_{P}=\Gamma \cap P, \Gamma_{L}=\Gamma_{L(P)}=v_{P}\left(\Gamma_{P}\right) \subset M_{P}$.

(8) $K_{P}=K \cap P, K_{L}=K_{L(P)}=v_{P}\left(K_{P}\right) \subset M_{P}, K_{P}^{\prime}=K^{\prime} \cap P=K_{P} A_{G}$.

The torus $\mathbf{S}_{\mathbf{P}}$ may also be identified as the greatest $\mathbb{Q}$-split torus in the center of $\mathbf{L}_{\mathbf{P}}$. It contains $\mathbf{S}_{\mathbf{G}}$ and we denote the quotient by $\mathbf{S}_{\mathbf{P}}^{\prime}=\mathbf{S}_{\mathbf{P}} / \mathbf{S}_{\mathbf{G}}$, with corresponding identity component $A_{P}^{\prime}=\mathbf{S}_{\mathbf{P}}^{\prime}(\mathbb{R})^{0}=A_{P} / A_{G}$. We identify $A_{P}^{\prime}$ with the subgroup $A_{P} \cap{ }^{0} G$ to obtain a canonical decomposition $A_{P}=A_{P}^{\prime} A_{G}$.

The group of real points of the Levi quotient is the direct product, $L_{P}=M_{P} \times A_{P}$. For any $x \in P$ write $v_{P}(x)=v_{M}(x) v_{A}(x)$ for its $M_{P}$ and its $A_{P}$ components and write $v_{A^{\prime}}(x)$ for the further projection of $v_{A}(x)$ to the quotient $A_{P}^{\prime}=A_{P} / A_{G}$. The group $P$ acts transitively on $D$ with isotropy $K_{P}^{\prime}=A_{G} K_{P}=\operatorname{Stab}_{P}\left(x_{0}\right)$.

The choice of standard basepoint $x_{0} \in D$ with associated Cartan involution $\theta: \mathbf{G} \rightarrow \mathbf{G}$ determines a unique $\theta$-stable lifting [BS], $\S 1.9, i=i_{x_{0}}: L_{P} \rightarrow P$. Denote the image by $L_{P}\left(x_{0}\right)=i\left(L_{P}\right)$. We obtain liftings of subgroups, $A_{P}\left(x_{0}\right)=i\left(A_{P}\right)$ and $M_{P}\left(x_{0}\right)=i\left(M_{P}\right)$. Thus the choice $x_{0} \in D$ of basepoint determines a canonical Langlands' decomposition

$$
P=\mathscr{U}_{P} A_{P}\left(x_{0}\right) M_{P}\left(x_{0}\right)
$$

and we write

$$
g=u_{g} a_{g} m_{g}
$$

where $u_{g}=g i v_{P}\left(g^{-1}\right), a_{g}=i v_{A}(g)$, and $m_{g}=i v_{M}(g)$ for any $g \in P$. The groups $K_{P} \subset P$ and $K_{L(P)}=v_{P}\left(K_{P}\right)$ are canonically isomorphic, in fact,

$$
K_{P}=i\left(K_{L(P)}\right) \subset M_{P}\left(x_{0}\right) \subset L_{P}\left(x_{0}\right) .
$$

By abuse of notation we will usually write $K_{P} \subset L_{P}$. If $x_{1} \in D$ is another basepoint with associated Cartan involution $\theta_{x_{1}}: \mathbf{G} \rightarrow \mathbf{G}$ then, by (2.1.2), the associated $\theta_{x_{1}}$-stable lifting $i_{x_{1}}: L_{P} \rightarrow P$ is given by

$$
i_{x_{1}}(y)=g i(y) g^{-1}
$$

where $g \in P$ is any element such that $g \cdot x_{0}=x_{1} \in D$. The geodesic action of Borel and Serre [BS], $\S 3$ is the right action of $A_{P}$ on $D$ which is given by 


$$
\left(z K_{P}^{\prime}\right) \cdot a=z i(a) K_{P}^{\prime} \in D=P / K_{P}^{\prime}
$$

for any $a \in A_{P}$ and $z \in P$. It is well defined since $i\left(A_{P}\right)$ commutes with $K_{P}^{\prime}$, and it passes to an action of $A_{P}^{\prime}=A_{P} / A_{G}$. The geodesic action commutes with the (left) action of $P$, and it is independent of the choice of basepoint, by (2.1.3). It is not an action by isometries.

For $g=u_{g} a_{g} m_{g} \in P$ as in (2.2.2), the element $a_{g} \in A_{P}$ is called the torus factor of $g$. We will often use without mention the following fact: if $\gamma=u_{\gamma} a_{\gamma} m_{\gamma} \in \Gamma \cap P$ then $a_{\gamma}=1$.

2.3. Roots. Fix once and for all a minimal rational parabolic subgroup $\mathbf{P}_{\mathbf{0}} \subset \mathbf{G}$. The parabolic subgroups $\mathbf{P} \supseteqq \mathbf{P}_{\mathbf{0}}$ are called standard. Let $\mathbf{S}_{0}=i\left(\mathbf{S}_{\mathbf{P}_{0}}\right)$ be the lift of $\mathbf{S}_{\mathbf{P}_{0}}$. Let $\Phi={ }_{\mathbb{Q}} \Phi\left(\mathbf{S}_{0}, \mathfrak{g}\right)$ denote the rational relative roots of $\mathfrak{g}$ with respect to $\mathbf{S}_{0}$. The unipotent radical $\mathscr{U}_{P_{0}}$ determines a linear order on the root system $\mathbb{Q}_{\mathbb{Q}} \Phi$ such that the positive roots are those occurring in $\mathfrak{R}_{0}=\operatorname{Lie}\left(\mathscr{U}_{P_{0}}\right)$. Let $\Delta$ denote the resulting collection of simple roots. Each subset $I \subset \Delta$ corresponds to a unique standard parabolic subgroup $\mathbf{P}_{0}(I) \supset \mathbf{P}_{0}$ ([BS], $\S 4,\left[\right.$ Bo3], §14.17, §21.11) such that $\mathbf{S}_{\mathbf{P}_{0}(I)} \subset \operatorname{ker}(\alpha)$ for all $\alpha \in I$.

Suppose $\mathbf{P} \subset \mathbf{G}$ is any rational parabolic subgroup. Then $\mathbf{P}$ is $\mathbf{G}(\mathbb{Q})$-conjugate to a unique standard parabolic subgroup $\mathbf{P}_{0}(I)$. Any choice of conjugating element $\mathbf{P}=g \mathbf{P}_{0}(I) g^{-1}$ gives rise to the same (canonical) isomorphism $\mathbf{S}_{\mathbf{P}} \cong \mathbf{S}_{\mathbf{P}_{0}(I)}$. The elements of $\Delta-I$ give rise (by conjugation and restriction to $\mathbf{S}_{\mathbf{P}}$ ) to the set $\Delta_{\mathbf{P}}$ of simple roots of $\mathbf{S}_{\mathbf{P}}$ occurring in $\mathfrak{N}_{P}$. The roots $\alpha \in \Delta_{P}$ are trivial on $\mathbf{S}_{\mathbf{G}}$ and form a basis for the character module $\chi_{\mathbb{Q}}\left(\mathbf{S}_{\mathbf{P}}^{\prime}\right)=\chi^{*}\left(\mathbf{S}_{\mathbf{P}} / \mathbf{S}_{\mathbf{G}}\right) \otimes_{\mathbb{Z}} \mathbb{Q}$. Rather than follow the common practice of identifying $\Delta_{P}$ with $\Delta-I$ we will, for any $\alpha \in \Delta_{P}$ denote by $\alpha_{0} \in \Delta$ the unique simple root which agrees with $\alpha$ after conjugation and restriction to $\mathbf{S}_{\mathbf{P}}$.

2.4. Two parabolic subgroups. If $\mathbf{P} \subset \mathbf{Q}$ are rational parabolic subgroups then $\overline{\mathbf{P}}=v_{Q}(\mathbf{P})$ is a rational parabolic subgroup of $\mathbf{L}_{\mathbf{Q}}$, with unipotent radical $\mathscr{U}_{\bar{P}}=\mathscr{U}_{P} / \mathscr{U}_{Q}$. The $\theta$-stable lifts of the Levi quotients satisfy $L_{P}\left(x_{0}\right) \subset L_{Q}\left(x_{0}\right)$ and we have a diagram

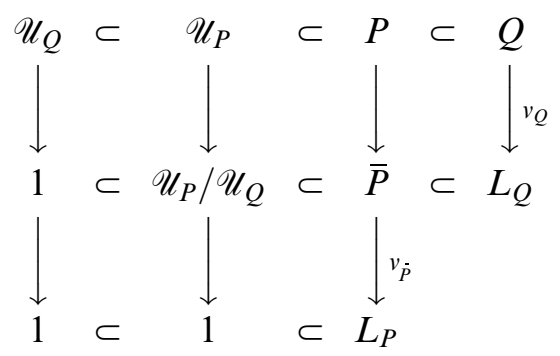

with $v_{Q} v_{\bar{P}}=v_{P}$. The inclusion $R_{d} \mathbf{Q} \subset R_{d} \mathbf{P}$ induces an injection $\mathbf{S}_{\mathbf{Q}} \hookrightarrow \mathbf{S}_{\mathbf{P}}$ which agrees with the inclusion $S_{P}\left(x_{0}\right) \supset S_{Q}\left(x_{0}\right)$. It follows from (2.4.4) below that the geodesic action of $A_{Q}^{\prime}$ on $D$ agrees with the restriction (to $A_{Q}^{\prime} \subset A_{P}^{\prime}$ ) of the geodesic action of $A_{P}^{\prime}$ on $D$ (cf. [BS], prop. 3.11). Each $\alpha \in \Delta_{Q}$ is the restriction to $\mathbf{S}_{\mathbf{Q}}$ of a unique simple root $i(\alpha) \in \Delta_{P}$. The association $i: \Delta_{Q} \rightarrow \Delta_{P}$ is injective, so $\Delta_{P}$ is the disjoint union

$$
\Delta_{P}=i\left(\Delta_{Q}\right) \amalg J \quad \text { with } \mathbf{S}_{\mathbf{Q}}=\left(\bigcap_{\alpha \in J} \operatorname{ker}(\alpha)\right)^{0} .
$$

Among rational parabolic subgroups containing $\mathbf{P}$, the group $\mathbf{Q}$ is determined by the set $J$, and we will write $\mathbf{Q}=\mathbf{P}(J)$. The subset $J \subset \Delta_{P}$ of simple roots may be identified with the set 


$$
J=\Delta_{\bar{P}}
$$

of simple roots $\Delta_{\bar{P}}$ of $\mathbf{S}_{\overline{\mathbf{P}}}^{\prime}=\mathbf{S}_{\overline{\mathbf{P}}} / \mathbf{S}_{\mathbf{Q}}$ occurring in $\mathfrak{N}_{\bar{P}}=\operatorname{Lie}\left(\mathscr{U}_{\bar{P}}\right)$. (Although the projection $v_{P}$ induces a canonical isomorphism $\mathbf{S}_{\mathbf{P}} \cong \mathbf{S}_{\overline{\mathbf{P}}}$, the torus $\mathbf{S}_{\mathbf{P}}$ corresponds to the parabolic subgroup $\mathbf{P} \subset \mathbf{G}$ so $\mathbf{S}_{\mathbf{P}}^{\prime}=\mathbf{S}_{\mathbf{P}} / \mathbf{S}_{\mathbf{G}}$ while $\mathbf{S}_{\overline{\mathbf{P}}}$ corresponds to the parabolic subgroup $\overline{\mathbf{P}} \subset \mathbf{L}_{\mathbf{Q}}$ so $\left.\mathbf{S}_{\overline{\mathbf{P}}}^{\prime}=\mathbf{S}_{\overline{\mathbf{P}}} / \mathbf{S}_{\mathbf{Q}} \cdot\right)$

A certain amount of confusion arises from the fact that $A_{Q}^{\prime}$ has two natural complements in $A_{P}^{\prime}$. One is the identity component $A_{Q^{\prime}}^{\prime}$ of the group of real points of the torus $\mathbf{S}_{\mathbf{Q}^{\prime}}^{\prime}=\mathbf{S}_{\mathbf{Q}^{\prime}} / \mathbf{S}_{\mathbf{G}}$ where

$$
\mathbf{S}_{\mathbf{Q}^{\prime}}=\left(\bigcap_{\alpha \in i\left(\Delta_{Q}\right)} \operatorname{ker}(\alpha)\right)^{0} \subset \mathbf{S}_{\mathbf{P}} .
$$

Then $\mathbf{S}_{\mathbf{Q}^{\prime}}$ is the (identity component of ) the center of the Levi quotient of the largest parabolic subgroup $\mathbf{Q}^{\prime} \supset \mathbf{P}$ such that $\mathbf{Q} \cap \mathbf{Q}^{\prime}=\mathbf{P}$, which we refer to as the parabolic subgroup containing $\mathbf{P}$ that is complementary to $\mathbf{Q}$. We therefore refer to the complementary decomposition $A_{P}^{\prime}=A_{Q^{\prime}}^{\prime} A_{Q^{\prime}}^{\prime}$. The other complement is

$$
A_{P}^{Q}\left(x_{0}\right)=A_{P}^{\prime} \cap M_{Q}\left(x_{0}\right)
$$

whose Lie algebra $\mathfrak{a}_{P}^{Q}$ is the orthogonal complement to $\mathfrak{a}_{Q}^{\prime}$ in $\mathfrak{a}_{P}^{\prime}$ with respect to any Weyl-invariant inner product on $\mathfrak{a}_{P}^{\prime}$. We will usually identify the quotient $A_{P} / A_{Q}=A_{P}^{\prime} / A_{Q}^{\prime}$ with this second complement, $A_{P}^{Q}$, and we will refer to the orthogonal decompositions $A_{P}^{\prime}=A_{Q}^{\prime} A_{P}^{Q}$ and $A_{P}=A_{Q} A_{P}^{Q}$.

The canonical Langlands decompositions of $P$ and $Q$ are related as follows: Set $\mathscr{U}_{\bar{P}}\left(x_{0}\right)=i_{x_{0}}\left(\mathscr{U}_{P} / \mathscr{U}_{Q}\right)$. Note that $M_{P}\left(x_{0}\right) \subset M_{Q}\left(x_{0}\right)$ and $A_{Q}=A_{Q}^{\prime} A_{G}$. If

$$
\bar{P}=\mathscr{U}_{\bar{P}}\left[A_{P}^{Q}\left(x_{0}\right) A_{G}\right] M_{P}\left(x_{0}\right)
$$

is the canonical Langlands decomposition of $\bar{P}$, then

$$
\begin{aligned}
P & =\left[\mathscr{U}_{Q} \mathscr{U}_{\bar{P}}\left(x_{0}\right)\right]\left[A_{Q}\left(x_{0}\right) A_{P}^{Q}\left(x_{0}\right)\right] M_{P}\left(x_{0}\right) \\
& =\mathscr{U}_{Q} A_{Q}\left(x_{0}\right)\left[\mathscr{U}_{\bar{P}}\left(x_{0}\right) A_{P}^{Q}\left(x_{0}\right) M_{P}\left(x_{0}\right)\right] .
\end{aligned}
$$

The first is the canonical Langlands decomposition of $P$ while the second is the decomposition of $P$ which is induced from the canonical Langlands decomposition of $Q$.

2.5. Boundary strata. Fix a rational parabolic subgroup $\mathbf{P} \subset \mathbf{G}$. Define

(1) the Borel-Serre boundary component $e_{P}=D / A_{P}^{\prime}$ (quotient under geodesic action),

(2) the Borel-Serre boundary stratum $Y_{P}=\Gamma_{P} \backslash e_{P}$,

(3) the reductive Borel-Serre boundary component

$$
D_{P}=\mathscr{U}_{P} \backslash e_{P}=P / K_{P} A_{P} \mathscr{U}_{P}=L_{P} / K_{P} A_{P}=M_{P} / K_{P} A_{G},
$$

(4) the reductive Borel-Serre boundary stratum $X_{P}=\Gamma_{P} \backslash D_{P}=\Gamma_{L(P)} \backslash D_{P}$.

The projection $v_{P}: \mathbf{P} \rightarrow \mathbf{L}_{P}$ induces a projection $\mu: e_{P} \rightarrow D_{P}$ which passes to a projection 
$\mu: Y_{P} \rightarrow X_{P}$. Writing $Y_{P}=\Gamma_{P} \backslash P / K_{P} A_{P}$ and $X_{P}=\Gamma_{L(P)} \backslash L_{P} / K_{P} A_{G}$, the mapping $\mu$ is just $\mu\left(\Gamma_{P} x K_{P} A_{P}\right)=\Gamma_{L(P)} v_{P}(x) K_{P} A_{G}$.

As in [Bo4], §4.2, the Langlands' decomposition (2.2.1) determines a (basepointdependent) diffeomorphism,

$$
F=F_{P}: \mathscr{U}_{P} \times A_{P}^{\prime} \times D_{P} \rightarrow D=P / K_{P}^{\prime}
$$

by

$$
F\left(u, a, m K_{L}\right)=u i_{x_{0}}(a) i_{x_{0}}(m) K_{P}
$$

where $u \in \mathscr{U}_{P}, a \in A_{P}^{\prime}$, and $m \in M_{P}$. With respect to the coordinates defined by the diffeomorphism $F$, the mapping $\mu$ is the projection to the third factor. The (left) action of $g \in P$ and the (right) geodesic action of $b \in A_{P}^{\prime}$ on $D$ are given by

$$
g \cdot\left(u, a, m K_{L}\right) \cdot b=\left(g u i_{x_{0}} v_{P}\left(g^{-1}\right), v_{A}(g) \cdot a b, v_{M}(g) \cdot m K_{L}\right)
$$

(where $u \in \mathscr{U}_{P}, a, b \in A_{P}^{\prime}$, and $m \in M_{P}$ ), as may be seen by applying the function $F$ to both sides of this equation. For any fixed $b \in A_{P}^{\prime}$ the set $F\left(\mathscr{U}_{P} \times\{b\} \times D_{P}\right) \subset D$ is called a $c a$ nonical cross section; it is a single orbit of the group

$$
{ }^{0} P=\bigcap_{\chi} \operatorname{ker}\left(\chi^{2}\right)=\mathscr{U}_{P} M_{P}
$$

the intersection being taken over all rationally defined characters $\chi: \mathbf{P} \rightarrow \mathbf{G L}_{\mathbf{1}}$. The pullback by $F$ of the canonical Riemannian metric on $D$ is given ([Bo4], §4.3) by the orthogonal sum,

$$
F^{*}\left(d s^{2}\right)=\sum_{\beta \in \Phi} a^{-2 \beta} h_{\beta}(z) \oplus d a^{2} \oplus d s_{M}^{2}
$$

where $d s_{M}^{2}$ is the canonical Riemannian metric on $D_{P}$ as determined by the Killing form for $M_{P}$, where $\Phi$ denotes the set of roots of $\mathscr{U}_{P}$ with respect to $A_{P}$, and $h_{\beta}(z)$ is a smoothly varying metric on the root space $\mathfrak{u}_{\beta}$.

2.6. The flat connection ([GHM], §7.10). For any point $x=g K_{P}^{\prime} \in D$ with $g=u a m$ decomposed according to (2.2.1), define the submanifold

$$
\mathscr{M}_{x}=F_{P}\left(\{u\} \times\{a\} \times D_{P}\right)=u \cdot i_{x_{0}}(a) . i_{x_{0}}\left(M_{P}\right) K_{P} \subset D .
$$

2.7. Lemma. The manifold $\mathscr{M}_{x}$ is perpendicular to the fibers of the mapping $v_{P}: D \rightarrow D_{P}$. The restriction $v_{P} \mid \mathscr{M}_{x}$ is an isometry. The submanifolds $\mathscr{M}_{x}$ form the horizontal submanifolds of a flat connection on the fiber bundle $\mu: e_{P} \rightarrow D_{P}$, which is independent of the choice of basepoint and is invariant under the action of $\Gamma_{P}$ and which therefore passes to a flat connection on $\mu: Y_{P} \rightarrow X_{P}$.

2.8. Proof. Perpendicularity follows from (2.5.4). Also, by (2.5.4), the mapping $v_{P}$ is an isometry. Finally the flat connection is $\Gamma_{P}$-invariant because by $(2.5 .3)$ the action of $\gamma \in \Gamma_{P}$ on $D$ is given by

$$
\gamma \cdot\left(u, a, m K_{L}\right)=\left(\gamma u i_{x_{0}} v_{P}\left(\gamma^{-1}\right), a, v_{M}(\gamma) \cdot m K_{L}\right)
$$

which does not mix the factors. 
2.9. Borel-Serre compactification. In this section we recall basic facts from [BS]. Let $\mathbf{P} \subset \mathbf{G}$ be a rational parabolic subgroup. The elements of $\Delta_{P}$ determine a canonical isomorphism $([\mathrm{BS}], \S 4.2) A_{P}^{\prime} \cong(0, \infty)^{\Delta_{P}}$ which extends to a unique partial compactification,

$$
\bar{A}_{P}^{\prime} \cong(0, \infty]^{\Delta_{P}}
$$

So each $\alpha \in \Delta_{P}$ extends to a homomorphism of semigroups $\alpha: \bar{A}_{P}^{\prime} \rightarrow(0, \infty]$. Denote by

$$
\begin{aligned}
& A_{P}^{\prime}(>1)=\left\{a \in A_{P}^{\prime} \mid \alpha(a)>1 \text { for all } \alpha \in \Delta_{P}\right\}, \\
& A_{P}^{\prime}(\geqq 1)=\left\{a \in A_{P}^{\prime} \mid \alpha(a) \geqq 1 \text { for all } \alpha \in \Delta_{P}\right\}
\end{aligned}
$$

and similarly for $\bar{A}_{P}^{\prime}(>1)$ and $\bar{A}_{P}^{\prime}(\geqq 1)$. The Borel-Serre partial compactification $\tilde{D}$ of $D$ is obtained by adjoining, for each rational parabolic subgroup $\mathbf{P} \subset \mathbf{G}$ the rational boundary component $e_{P}=D / A_{P}^{\prime}$ as the set of limits of the $A_{P}^{\prime}$ geodesic orbits in $D$, together with the Satake topology [Sat], §2 (p. 562), [BS], §7.1, [Z3], §3.7. It is covered by "corners"; the corner associated to $P$ is

$$
D(P)=D \times_{A_{P}^{\prime}} \bar{A}_{P}^{\prime}=\coprod_{Q \supseteqq P} e_{Q}
$$

Then $D(P)$ is an open $\mathbf{P}(\mathbb{Q})$-invariant neighborhood (in $\tilde{D})$ of the boundary component $e_{P}$, on which $\mathbf{P}(\mathbb{Q})$ acts in a continuous and component-preserving way. The diffeomorphism $F$ of equation (2.5.2) extends to a diffeomorphism of manifolds with corners,

$$
\bar{F}: \mathscr{U} \times \bar{A}_{P} \times D_{P} \cong D(P) .
$$

The action $T_{g}: D \rightarrow D$ of any $g \in \mathbf{G}(\mathbb{Q})$ extends continuously to a mapping

$$
\tilde{T}_{g}: \tilde{D} \rightarrow \tilde{D}
$$

which takes the neighborhood $D(P)$ of $e_{P}$ isomorphically to the neighborhood $D\left({ }^{g} P\right)$ of $e_{q} P$ (where ${ }^{g} P=g P g^{-1}$ ). (The proof of this fact is recalled in $\S 6.3, \S 6.4$.) It follows that the Borel-Serre compactification $\tilde{X}=\Gamma \backslash \tilde{D}$ is a (compact) manifold with corners, stratified with one stratum $Y_{P}=\Gamma_{P} \backslash e_{P}$ for each $\Gamma$-conjugacy class of rational parabolic subgroups $\mathbf{P}$. The real analytic structure on $D$ extends to a semi-analytic structure on $\tilde{D}$ and passes to a subanalytic structure on $\tilde{X}$. Denote by $\tilde{\tau}: \tilde{D} \rightarrow \tilde{X}$ the natural projection.

2.10. Reductive Borel-Serre compactification. The reductive Borel-Serre partial compactification $\bar{D}$ of $D$ was first described in $[\mathrm{Z1}], \S 4.2$, p. 190; see also [GHM], §8. It is the topological space obtained by collapsing each boundary component $e_{P}$ in $\tilde{D}$ to its reductive quotient $D_{P}(\S 2.5)$, together with the quotient topology. (See also [Z3], §3.7.) The geodesic projection

$$
\pi_{P}: D \rightarrow D_{P}
$$

is the composition $D \rightarrow e_{P} \rightarrow D_{P}$. The closure $\bar{D}_{P}$ of $D_{P}$ in $\bar{D}$ is the reductive Borel-Serre partial compactification of $D_{P}$. Let $\mu: \tilde{D} \rightarrow \bar{D}$ denote the quotient mapping: it is continuous, its restriction to $D$ is the identity, and its restriction to each boundary stratum agrees with the projection $\mu: Y_{P} \rightarrow X_{P}$ of $\S 2.5$. Define 


$$
D[P]=\mu(D(P))=\bigcup_{Q \supseteqq P} D_{Q}
$$

to be the image of the corner associated to $P$ : it is an open $\mathbf{P}(\mathbb{Q})$-invariant neighborhood of $D_{P}$ in $\bar{D}$ on which $\mathbf{P}(\mathbb{Q})$ acts in a component-preserving way. The action $T_{g}: \tilde{D} \rightarrow \tilde{D}$ (2.9.5) of any $g \in \mathbf{G}(\mathbb{Q})$ passes to a mapping $\bar{T}_{g}: \bar{D} \rightarrow \bar{D}$ which takes the neighborhood $D[P]$ of $D_{P}$ isomorphically to the neighborhood $D\left[{ }^{g} P\right]$ of $D_{g_{P}}$. It follows that the reductive Borel-Serre compactification

$$
\bar{X}=\Gamma \backslash \bar{D}
$$

is a compact singular space, canonically stratified with one boundary stratum $X_{P}=\Gamma_{P} \backslash D_{P}$ for each $\Gamma$-conjugacy class of rational parabolic subgroups $\mathbf{P} \subset \mathbf{G}$. The closure $\bar{X}_{P}$ of $X_{P}$ in $\bar{X}$ is the reductive Borel-Serre compactification of $X_{P}$. There are $\left|\Delta_{P}\right|$ maximal boundary strata $X_{Q}$ such that $\bar{X}_{Q} \supset \bar{X}_{P}$, each corresponding to a maximal (rational) parabolic subgroup $\mathbf{Q}=\mathbf{P}\left(\Delta_{P}-\{\alpha\}\right)$ for $\alpha \in \Delta_{P}$ (cf. §2.4). Then $\bar{X}_{P}$ is the intersection

$$
\bar{X}_{P}=\bigcap_{Q} \bar{X}_{Q}
$$

of these $\left|\Delta_{P}\right|$ maximal boundary strata.

It is not difficult to see ([Bo3], §11.7 (iii)) that if $\mathbf{P}$ and $\mathbf{P}^{\prime}$ are $\mathbf{G}(\mathbb{Q})$-conjugate but are not $\Gamma$-conjugate, then

$$
\bar{X}_{P} \cap \bar{X}_{P^{\prime}}=\emptyset .
$$

The identity mapping $X \rightarrow X$ extends uniquely to a continuous surjection $\mu: \tilde{X} \rightarrow \bar{X}$ and the subanalytic structure on $\tilde{X}$ passes to a subanalytic structure on $\bar{X}$. Denote by $\bar{\tau}: \bar{D} \rightarrow \bar{X}$ the projection. Define $X(P)=\tilde{\tau}(D(P))$ and $X[P]=\bar{\tau}(D[P])$. The following diagram may be useful in helping to sort out these spaces:

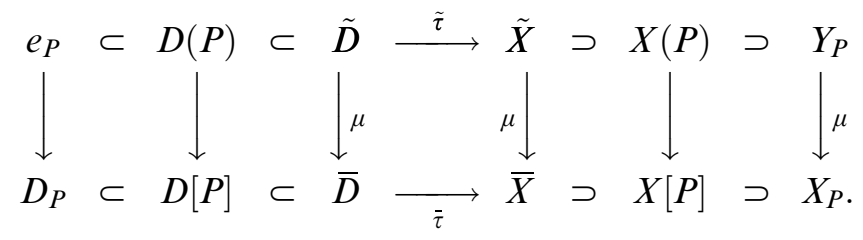

\section{Parabolic neighborhoods and root functions}

As in $\$ 2, \mathbf{G}$ denotes a connected linear reductive algebraic group defined over $\mathbb{Q}, D$ denotes the associated symmetric space, $K^{\prime}=A_{G} K\left(x_{0}\right)$ is the stabilizer in $G$ of a fixed basepoint $x_{0} \in D, \Gamma \subset \mathbf{G}(\mathbb{Q})$ is an arithmetic group and $X=\Gamma \backslash D$. Although the constructions in this section refer to the reductive Borel-Serre compactification $\bar{X}$ of $X$ (and the reductive Borel-Serre partial compactification $\bar{D}$ of $D$ ), they may just as well be applied to the BorelSerre compactification $\tilde{X}$ (and the Borel-Serre partial compactification $\tilde{D}$ of $D$ ). Rather than repeat each statement for both compactifications, we will present the RBS case only.

3.1. Parabolic neighborhoods. Let $\mathbf{P} \subset \mathbf{G}$ be a rational parabolic subgroup. Let $\alpha: \bar{D} \rightarrow \Gamma_{P} \backslash \bar{D}$ and $\beta: \Gamma_{P} \backslash \bar{D} \rightarrow \Gamma \backslash \bar{D}=\bar{X}$ be the projections. We say that an open set $V \subset \bar{D}$ is $\Gamma$-parabolic (with respect to $\mathbf{P}$ ) if 
(1) it is invariant under the geodesic action of the semigroup $A_{P}(\geqq 1)(2.9 .2)$ and

(2) if $\gamma \in \Gamma$ and $\gamma V \cap V \neq \emptyset$ then $\gamma \in \Gamma \cap P$.

Item (2) means that the covering $\beta: \Gamma_{P} \backslash \bar{D} \rightarrow \bar{X}$ is one to one on the $\alpha(V)$ so it takes $\alpha(V)$ homeomorphically to its image $\bar{\tau}(V) \subset \bar{X}$. In this case we will also refer to $\alpha(V) \subset \Gamma_{P} \backslash \bar{D}($ resp. $\bar{\tau}(V) \subset \bar{X})$ as $\Gamma$-parabolic open sets.

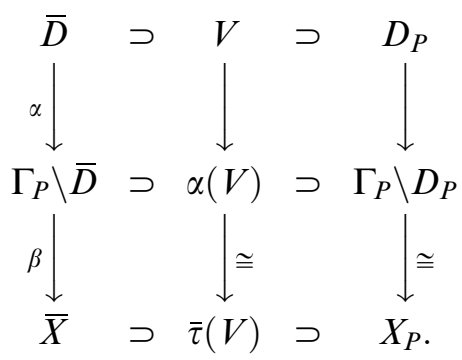

Every stratum $X_{P}$ admits a fundamental system of $\Gamma$-parabolic neighborhoods. In section 4.1 we will review a theorem of Saper [Sa] (thm. 8.1) which states the stronger fact that the closure $\bar{X}_{P}$ of each stratum $X_{P} \subset \bar{X}$ admits a fundamental system of $\Gamma$-parabolic neighborhoods.

3.2. Root functions. Let $\mathbf{P} \subset \mathbf{G}$ be a rational parabolic subgroup. Each character $\alpha \in \chi_{\mathbb{Q}}^{*}\left(\mathbf{S}_{\mathbf{P}}^{\prime}\right)$ determines a mapping

$$
f_{\alpha}^{P}: D \rightarrow \mathbb{R}_{>0}
$$

by $f_{\alpha}^{P}\left(F\left(u, a, m K_{L}\right)\right)=\alpha(a)$ using (2.5.2). The mapping $f_{\alpha}^{P}$ is independent of the choice of basepoint. For any $g^{\prime}=u^{\prime} a^{\prime} m^{\prime} \in P$, any $b^{\prime} \in A_{P}^{\prime}$, any $\gamma \in \Gamma \cap P$, and any $x \in D$ we have

$$
f_{\alpha}^{P}\left(\gamma g^{\prime} x \cdot b^{\prime}\right)=\alpha\left(a^{\prime} b^{\prime}\right) f_{\alpha}^{P}(x) .
$$

If $\alpha \in \Delta_{P}$ is a simple root, we say $f_{\alpha}^{P}$ is a root function. If $\gamma \in \Gamma, \mathbf{P}^{\prime}=\gamma \mathbf{P} \gamma^{-1}$ and if $\alpha^{\prime} \in \Delta_{P^{\prime}}$ is the root corresponding to $\alpha \in \Delta_{P}$ then, for all $x \in D$,

$$
f_{\alpha^{\prime}}^{P^{\prime}}(\gamma x)=f_{\alpha}^{P}(x)
$$

The root function $f_{\alpha}^{P}: D \rightarrow(0, \infty)$ extends to a continuous function $D[P] \rightarrow(0, \infty)$ (cf. $\S 3.5$ below) which passes to a function $\Gamma_{P} \backslash D[P] \rightarrow(0, \infty]$ whose restriction to any $\Gamma$-parabolic neighborhood $U \subset \bar{X}$ of $X_{P}$ we also denote by

$$
f_{\alpha}^{P}: U \rightarrow(0, \infty]
$$

Similarly the geodesic projection $\pi_{P}: D \rightarrow D_{P}$ (cf. (2.10.1)) extends continuously to a projection $D(P) \rightarrow D_{P}$ and passes to projections $D[P] \rightarrow D_{P}$ and $\Gamma_{P} \backslash D[P] \rightarrow \Gamma_{P} \backslash D$ whose restriction to any parabolic neighborhood $U \subset \bar{X}$ we denote by

$$
\pi_{P}: U \rightarrow X_{P}
$$

The following lemma is a straightforward consequence of the definitions. 
3.3. Lemma. Let $U \subset \bar{X}$ be a parabolic neighborhood of the stratum $X_{P}$. Let $\left\{x_{n}\right\} \subset U$ be a sequence of points and let $y \in X_{P}$. The sequence $\left\{x_{n}\right\}$ converges to $y$ in $\bar{X}$ if and only if the following hold:

(1) $\pi_{P}\left(x_{n}\right) \rightarrow y$ in $X_{P}$ and

(2) $f_{\alpha}^{P}\left(x_{n}\right) \rightarrow \infty$ for all $\alpha \in \Delta_{P}$.

3.4. Suppose $\mathbf{Q} \supset \mathbf{P}$ is another rational parabolic subgroup of $\mathbf{G}$, corresponding, say, to a subset $J \subset \Delta_{P}$ with $\mathbf{S}_{\mathbf{Q}} \subset \operatorname{ker}(\alpha)$ for all $\alpha \in J$, so that $\Delta_{P}=i\left(\Delta_{Q}\right) \amalg J$ as in (2.4.1). Let $\bar{P}=v_{Q}(P) \subset L_{Q}$ be the resulting parabolic subgroup of $L_{Q}$. It acts transitively on the boundary component $D_{Q}$.

Let $x \in D$, say $x=u_{Q} a_{Q}^{\prime} a_{G} u_{\bar{P}} a_{P}^{Q} m_{P} K_{P}^{\prime}$ is decomposed according to (2.4.5) with $a_{Q}=a_{Q}^{\prime} a_{G}$. Then $\pi_{Q}(x)=u_{\bar{P}} a_{P}^{Q} a_{G} m_{P} K_{P}^{\prime} \in \bar{P} / K_{P}^{\prime}=D_{Q}$ so the following equations hold:

$$
\begin{aligned}
& f_{\alpha}^{P}(x)=\alpha\left(a_{Q}^{\prime} a_{P}^{Q}\right) \text { for all } \alpha \in \Delta_{P}, \\
& f_{\beta}^{Q}(x)=i(\beta)\left(a_{Q}^{\prime}\right) \text { for all } \beta \in \Delta_{Q}, \\
& f_{\alpha}^{\bar{P}}\left(\pi_{Q}(x)\right)=f_{\alpha}^{P}(x)=\alpha\left(a_{P}^{Q}\right) \quad \text { for all } \alpha \in J=\Delta_{\bar{P}},
\end{aligned}
$$

since $\alpha\left(a_{Q}^{\prime}\right)=1$ for all $\alpha \in J$. From this we may conclude:

3.5. Proposition. For all $\alpha \in \Delta_{P}$, the root function $f_{\alpha}^{P}$ extends continuously to a function $f_{\alpha}^{P}: D[P] \rightarrow(0, \infty]$ such that, for all $x \in D$ we have

$$
f_{\alpha}^{P}\left(\pi_{Q}(x)\right)= \begin{cases}f_{\alpha}^{P}(x) & \text { for } \alpha \in J, \\ \infty & \text { for } \alpha \in \Delta_{P}-J .\end{cases}
$$

The boundary component $D_{Q} \subset D[P]$ is the set of $x \in D[P]$ such that:

$$
\begin{cases}f_{\alpha}^{P}(x)=\infty & \text { for all } \alpha \in \Delta_{P}-J, \\ f_{\alpha}^{P}(x)<\infty & \text { for all } \alpha \in J .\end{cases}
$$

3.6. Remarks. Of course similar statements apply to the root function $f_{\alpha}^{P}: U \rightarrow(0, \infty]$ for any parabolic neighborhood $U \subset \bar{X}$ of $X_{P}$. We think of the "negative gradient" of the root functions $f_{\alpha}^{P}$ as pointing in the "normal directions" to $X_{P}$. For $\alpha \in J$, $-\operatorname{grad} f_{\alpha}^{P}$ points from $X_{P}$ "into" $X_{Q}$.
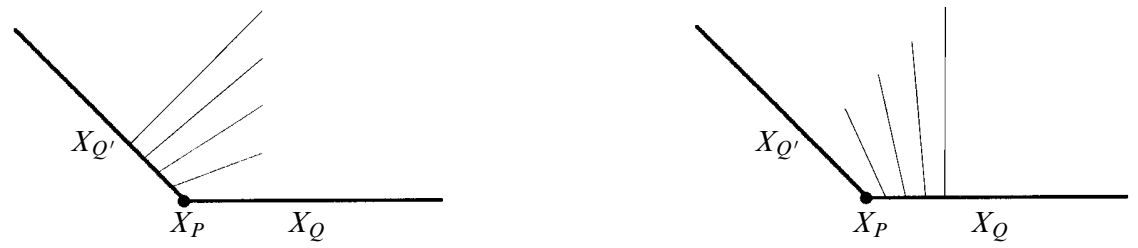

Figure 1. Level curves of $f_{\alpha}^{P}$ for $\alpha \in i\left(\Delta_{Q}\right)$ and $\alpha \in J$ respectively. 
Zucker's vexatious point ([Z1], §3.19) is that for $\mathbf{P} \subset \mathbf{Q}$ and for $\beta \in \Delta_{Q}$, the root functions $f_{\beta}^{Q}$ and $f_{i(\beta)}^{P}$ do not necessarily agree: see (3.4.1) and (3.4.2) above. (In fact, they agree precisely if $i(\beta)\left(a_{P}^{Q}\right)=1$, which is to say, if $A_{Q^{\prime}}^{\prime}$ and $A_{Q}^{\prime}$ are orthogonal, where $\mathbf{Q}^{\prime} \supset \mathbf{P}$ is the parabolic subgroup complementary to $\mathbf{Q}$.) The nature of the level sets of $f_{\beta}^{Q}$ are depicted in Figure 2.

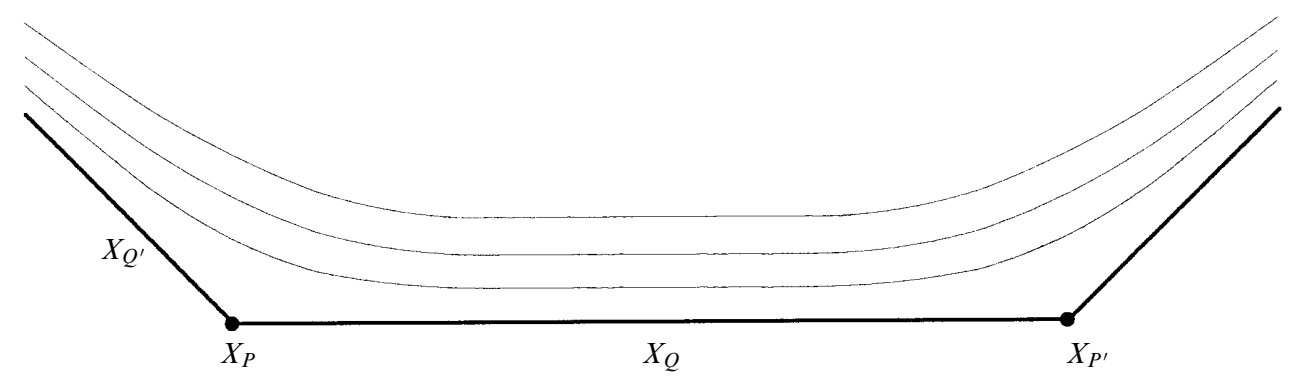

Figure 2. Level sets of $f_{\beta}^{Q}$.

This shortcoming will be circumvented by replacing the root function $f_{\alpha}^{P}$ with Saper's partial distance function $r_{\alpha}^{P}$ (associated to a tiling), which is patched together from the various relevant root functions; cf. (4.3.2).

\section{Tilings}

In this section we recall a construction of Saper [Sa]. An equivalent construction of Leuzinger [Le1] could be used instead. See also [Ar2] and [L2].

4.1. Tilings of $\overline{\boldsymbol{D}}$. As in $\S 2$, G denotes a connected linear reductive algebraic group defined over $\mathbb{Q}, D$ denotes the associated symmetric space, $K^{\prime}=A_{G} K\left(x_{0}\right)$ is the stabilizer in $G$ of a fixed basepoint $x_{0} \in D, \Gamma \subset \mathbf{G}(\mathbb{Q})$ is an arithmetic group and $X=\Gamma \backslash D$. Let $\mathscr{P}_{1}$ denote the set of proper maximal rational parabolic subgroups of $\mathbf{G}$. For each $\mathbf{Q} \in \mathscr{P}_{1}$ choose $b_{Q} \in A_{Q}^{\prime}$. The collection $\mathbf{b}=\left\{b_{Q}\right\}$ of such choices is called a parameter, the set of which we denote by $\mathscr{B}$. The parameters are partially ordered with $\mathbf{b} \leqq \mathbf{c}$ iff $\alpha_{Q}\left(b_{Q}\right) \leqq \alpha_{Q}\left(c_{Q}\right)$ for all $\mathbf{Q} \in \mathscr{P}_{1}$, where $\Delta_{Q}=\left\{\alpha_{Q}\right\}$ is the simple root associated with the maximal parabolic subgroup $\mathbf{Q}$. A choice $\mathbf{b} \in \mathscr{B}$ of parameter determines, for any rational parabolic subgroup $\mathbf{P} \subset \mathbf{G}$ a unique element $b_{P} \in A_{P}^{\prime}$ such that, for each rational maximal parabolic subgroup $\mathbf{Q} \supset \mathbf{P}$, the element $b_{P} b_{Q}^{-1}$ lies in $A_{P}^{Q}$ (cf. $\$ 2.4$ ). In other words, $\log \left(b_{Q}\right)$ is the orthogonal projection of $\log \left(b_{P}\right) \in \mathfrak{a}_{P}^{\prime}$ with respect to any Weyl-invariant inner product on $\mathfrak{a}_{P}^{\prime}$.

Recall from [Sa] that a tiling with parameter $\mathbf{b} \in \mathscr{B}$ is a cover of the reductive BorelSerre partial compactification

$$
\bar{D}=\coprod_{\mathbf{P} \in \mathscr{P}} D^{P}
$$

by disjoint sets (called tiles) such that:

(1) The central tile $D^{0}=D^{G}$ is a closed, codimension 0 submanifold with 
corners contained in $D$. Its closed boundary faces $\left\{\partial^{P} D^{0}\right\}$ are indexed by $\mathbf{P} \in \mathscr{P}$ with $\mathbf{P} \subset \mathbf{Q} \Leftrightarrow \partial^{P} D^{0} \subset \partial^{Q} D^{0}$.

(2) Each boundary face $\partial^{P} D^{0}$ is contained in the "cross section" $F\left(\mathscr{U}_{P} \times\left\{b_{P}\right\} \times D_{P}\right)$ where $F$ is defined in (2.5.2).

(3) Each tile $D^{P}=\partial^{P} D^{0} \cdot \bar{A}_{P}(>1)$ is obtained from $\partial^{P} D^{0}$ by flowing out under the geodesic action of the cone $\bar{A}_{P}(>1)$ (cf. (2.9.1)).

For any rational parabolic subgroup $\mathbf{Q}$, the intersections $\left\{D^{P} \cap \bar{D}_{Q}\right\}$ (over all rational parabolic subgroups $\mathbf{P} \subseteq \mathbf{Q})$ form a tiling of the reductive Borel-Serre partial compactification $\bar{D}_{Q}$, whose central tile we denote by

$$
D_{Q}^{0}=D^{Q} \cap D_{Q}
$$

Then the tile $D^{Q}$ is given by

$$
D^{Q}=\left\{x \in D[Q] \subset \bar{D} \mid \pi_{Q}(x) \in D_{Q}^{0} \text { and } f_{\alpha}^{Q}(x)>\alpha\left(b_{Q}\right) \forall \alpha \in \Delta_{Q}\right\}
$$

and the boundary face $\partial^{Q} D^{0}$ is

$$
\partial^{Q} D^{0}=\left\{x \in D \mid \pi_{Q}(x) \in D_{Q}^{0} \text { and } f_{\alpha}^{Q}(x)=\alpha\left(b_{Q}\right) \forall \alpha \in \Delta_{Q}\right\} .
$$

A tiling, if it exists, is uniquely determined by its parameter $\mathbf{b} \in \mathscr{B}$, in which case we say that the parameter is regular. The parameter $\mathbf{b}$ is $\Gamma$-invariant if, for all $\gamma \in \Gamma$, we have $b_{\gamma Q \gamma^{-1}}=a_{\gamma Q \gamma^{-1}}\left(\gamma x_{0}\right) b_{Q}$. The tiling $\left\{D^{Q}\right\}$ is $\Gamma$-invariant if $\gamma D^{P}=D^{\gamma P \gamma^{-1}}$ for all $\gamma \in \Gamma$. A tiling is $\Gamma$-invariant if and only if its parameter $\mathbf{b}$ is $\Gamma$-invariant ([Sa], Corollary 2.7). In $[\mathrm{Sa}]$, Thm. 10.1, Saper proves the following.

4.2. Theorem. If the tiling parameter $\mathbf{b} \in \mathscr{B}$ is chosen sufficiently large (with respect to the above partial ordering) and $\Gamma$-invariant, then there exists a unique tiling with parameter $\mathbf{b} \in \mathscr{B}$, and it is $\Gamma$-invariant. Moreover, for any $\mathbf{Q} \in \mathscr{P}$ the union

$$
T\left(\bar{D}_{Q}\right)=\coprod_{P \cong Q} D^{P}
$$

is an open $\Gamma_{Q}$-invariant parabolic neighborhood of $\bar{D}_{Q}$ in $\bar{D}$ which may be made arbitrarily small by choosing the parameter $\mathbf{b}$ sufficiently large.

Henceforth we shall refer to such a parameter as regular and sufficiently large. Fix such a parameter $\mathbf{b}=\left\{b_{Q}\right\}$. Denote the closure of $T\left(\bar{D}_{Q}\right)$ by $\bar{T}\left(\bar{D}_{Q}\right)$, and the boundary by $\partial T\left(\bar{D}_{Q}\right)=\bar{T}\left(\bar{D}_{Q}\right)-T\left(\bar{D}_{Q}\right)$. Following [Sa], Thm. 8.1 (ii), for each $\alpha \in \Delta_{Q}$, define the partial distance function $r_{\alpha}^{Q}: \bar{T}\left(\bar{D}_{Q}\right) \rightarrow[0,1]$ by

$$
r_{\alpha}^{Q}(x)= \begin{cases}f_{\alpha}^{Q}(x)^{-1} \alpha\left(b_{Q}\right) & \text { for } x \in \bar{D}^{Q}, \\ f_{i(\alpha)}^{P}(x)^{-1} i(\alpha)\left(b_{P}\right) & \text { for } x \in \bar{D}^{P},\end{cases}
$$

whenever $\mathbf{P} \subset \mathbf{Q}$. Here, $i: \Delta_{Q} \hookrightarrow \Delta_{P}$ is the inclusion (2.4.1) and $\bar{D}^{Q}$ (resp. $\bar{D}^{P}$ ) is the closure of the tile $D^{Q}\left(\right.$ resp. $\left.D^{P}\right)$. 
4.3. Lemma. The following statements hold.

(1) The mapping $r_{\alpha}^{Q}: \bar{T}\left(\bar{D}_{Q}\right) \rightarrow[0,1]$ is well-defined, continuous, and piecewise analytic.

(2) For all $\alpha \in \Delta_{Q}$, the geodesic action by $t \in A_{Q}(\geqq 1)$ satisfies

$$
r_{\alpha}^{Q}(x \cdot t)=r_{\alpha}^{Q}(x) \alpha(t)^{-1}
$$

whenever $x \in \bar{T}\left(\bar{D}_{Q}\right)$.

(3) If $x \in \bar{T}\left(\bar{D}_{Q}\right)$ then

$$
\begin{gathered}
x \in \bar{D}_{Q} \Leftrightarrow r_{\alpha}^{Q}(x)=0 \text { for all } \alpha \in \Delta_{Q}, \\
x \in \partial T\left(\bar{D}_{Q}\right) \Leftrightarrow r_{\alpha}^{Q}(x)=1 \text { for some } \alpha \in \Delta_{Q} .
\end{gathered}
$$

(4) If $\gamma \in \Gamma \cap Q$ then $r_{\alpha}^{Q}(\gamma x)=r_{\alpha}^{Q}(x)$. then

(5) If $\gamma \in \Gamma$ and $\mathbf{Q}^{\prime}=\gamma \mathbf{Q} \gamma^{-1}$ and if $\alpha^{\prime} \in \Delta_{\mathbf{Q}^{\prime}}$ is the simple root corresponding to $\alpha \in \Delta_{\mathbf{Q}}$

$$
r_{\alpha^{\prime}}^{Q^{\prime}}(\gamma x)=r_{\alpha}^{Q}(x)
$$

(6) If $\mathbf{P} \subset \mathbf{Q}$ and if $\Delta_{P}=i\left(\Delta_{Q}\right) \amalg J$ as in (2.4.1) then, for all $\alpha \in \Delta_{Q}$ and for all $x \in T\left(\bar{D}_{P}\right) \subset T\left(\bar{D}_{Q}\right)$ we have

$$
r_{i(\alpha)}^{P}(x)=r_{\alpha}^{Q}(x)
$$

4.4. Proof. As in $\$ 2.4$, write $A_{P}^{\prime}=A_{Q}^{\prime} A_{P}^{Q}$ with $\mathfrak{a}_{Q}^{\prime}$ the orthogonal complement to $\mathfrak{a}_{P}^{Q}$ in $\mathfrak{a}_{P}^{\prime}$. So the elements $b_{P}$ and $b_{Q}$ determined by the parameter $\mathbf{b}$ satisfy $b_{P}=b_{Q} b_{P}^{Q}$ for some $b_{P}^{Q} \in A_{P}^{Q}$. Now suppose that $x=u_{Q} a_{Q}^{\prime} a_{G} u_{\bar{P}} a_{P}^{Q} m_{P} K_{P} \in P / K_{P}=D$ is decomposed according to (2.4.5). Set $a_{P}^{\prime}=a_{Q}^{\prime} a_{P}^{Q} \in A_{P}^{\prime}$. If $x \in \partial^{P} D^{0}$ then by property (2), $a_{P}^{\prime}=b_{P}$, that is, $a_{Q}^{\prime}=b_{Q}$ and $a_{P}^{Q}=b_{P}^{Q}$. Flowing out under the geodesic action of $A_{Q}^{\prime}$ we see that $x \in \bar{D}^{Q} \cap \bar{D}^{P} \cap D$ implies that $a_{P}^{Q}=b_{P}^{Q}$. For such a point $x$ and for each $\alpha \in \Delta_{Q}$, we have

$$
\begin{aligned}
f_{i(\alpha)}^{P}(x)^{-1} i(\alpha)\left(b_{P}\right) & =i(\alpha)\left(a_{Q} a_{P}^{Q}\right)^{-1} i(\alpha)\left(b_{Q} b_{P}^{Q}\right) \\
& =\alpha\left(a_{Q}^{-1} b_{Q}\right)=f_{\alpha}^{Q}(x)^{-1} \alpha\left(b_{Q}\right)
\end{aligned}
$$

so both equations (4.2.2) agree on their common domain of definition, proving (1).

By continuity, it suffices to prove (2) for points $x \in T\left(\bar{D}_{Q}\right)$. The geodesic action by $t \in A_{Q}(\geqq 1)$ preserves the tiles in $T\left(\bar{D}_{Q}\right)$ so $(2)$ may be checked tile by tile. If $x \in D^{Q}$ then $f_{\alpha}^{Q}(x \cdot t)=f_{\alpha}^{Q}(x) \alpha(t)$ by (3.2.2). If $x \in D^{P}$ for some $\mathbf{P} \subset \mathbf{Q}$, write $\Delta_{P}=i\left(\Delta_{Q}\right) \amalg J$ as in (2.4.1), note that $A_{Q}(\geqq 1) \subset A_{P}(\geqq 1)$ and compute, for $\alpha \in \Delta_{Q}$,

$$
f_{i(\alpha)}^{P}(x \cdot t)=f_{i(\alpha)}^{P}(x) i(\alpha)(t)=f_{i(\alpha)}^{P}(x) \alpha(t)
$$


which proves the second statement. Part (3) follows from (3.2.2) for points $x \in \bar{T}_{Q}$ and from (3.2.3) for points $x \in \bar{T}_{P}$. Part (4) follows from Lemma 3.3 for points $x \in \bar{T}_{Q}$ and from (3.5.1) for points $x \in \bar{T}_{P}$. Part (5) follows from (3.2.3) and part (6) is an immediate consequence of the definition.

4.5. Tiling of $\overline{\boldsymbol{X}}$. Suppose $\mathbf{b} \in \mathscr{B}$ is a sufficiently large regular parameter and (4.1.1) is the associated $\Gamma$-invariant tiling. Let $\bar{\tau}: \bar{D} \rightarrow \bar{X}$ denote the projection to the reductive Borel-Serre compactification of $X=\Gamma \backslash D$. If $\mathbf{P}, \mathbf{P}^{\prime}$ are rational parabolic subgroups of $\mathbf{G}$ then either $\bar{\tau}\left(D^{P}\right) \cap \bar{\tau}\left(D^{P^{\prime}}\right)=\emptyset$ or else they coincide. Hence the collection of images

$$
X^{P}=\bar{\tau}\left(D^{P}\right)
$$

forms a decomposition of $\bar{X}$ whose "tiles" are indexed by the set of $\Gamma$-conjugacy classes of rational parabolic subgroups of $\mathbf{G}$. Let $X_{P}^{0}=X^{G}=\bar{\tau}\left(D_{P}^{0}\right)$ be the "central tile." Denote by

$$
T\left(\bar{X}_{P}\right)=\bar{\tau}\left(T\left(\bar{D}_{P}\right)\right)=\coprod_{\{R\} \cong P} X^{R}
$$

the resulting neighborhood of $\bar{X}_{P}$ in $\bar{X}$, and by $\partial T\left(\bar{X}_{P}\right)=\bar{T}\left(\bar{X}_{P}\right)-T\left(\bar{X}_{P}\right)$ its boundary. (Here, $R$ runs through a set of representatives, one from each $\Gamma$-conjugacy class $\{R\}$ of parabolic subgroups contained in $P$.) For all $\alpha \in \Delta_{P}$ the functions $r_{\alpha}^{P}$ pass to piecewise analytic functions on $T\left(\bar{X}_{P}\right)$, which we also denote by $r_{\alpha}^{P}$.

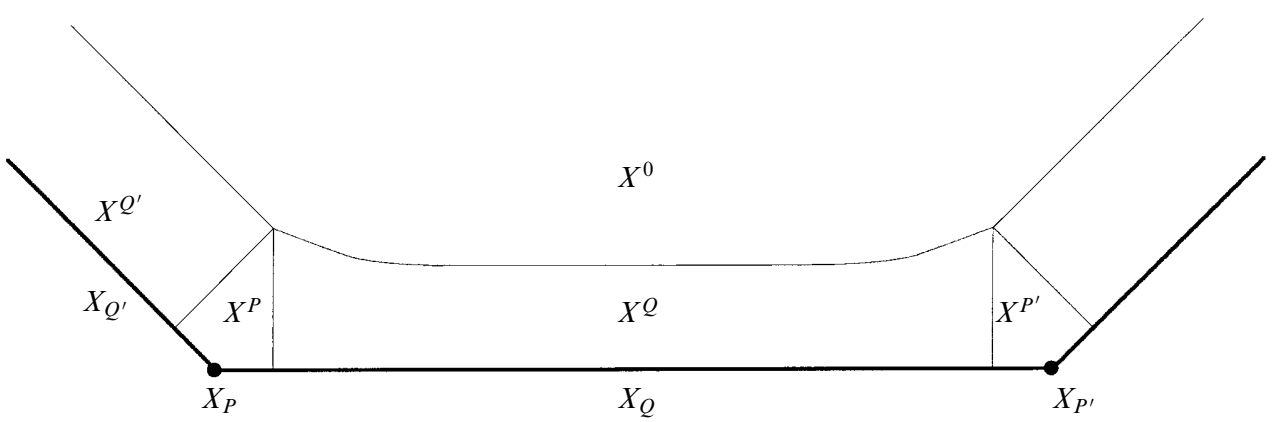

Figure 3. Tiles.

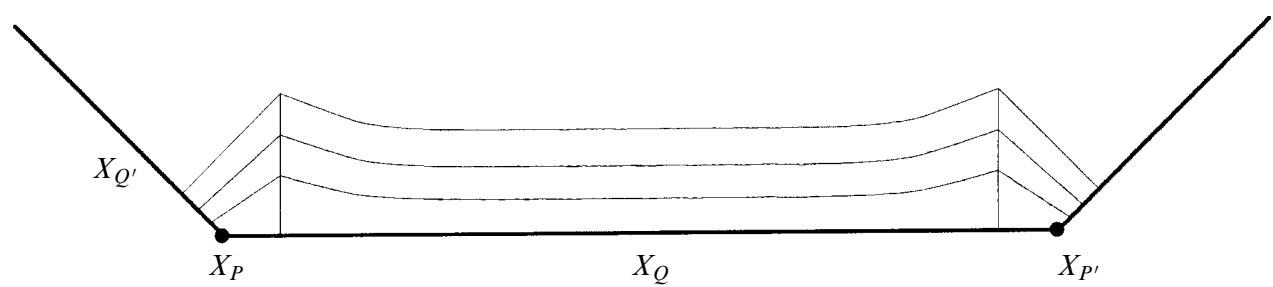

Figure 4. Level sets of $r_{\alpha}^{Q}$.

4.6. Retraction and exhaustion. Saper proves $([\mathrm{Sa}], \S 6.1)$ that there exists a unique $\Gamma$-equivariant continuous and piecewise analytic "geodesic" retraction $R: D \rightarrow D^{0}$ which is the identity on $D^{0}$ such that, for all $y \in D^{P}$ and for all $t \in A_{P}(\geqq 1)$ the following holds:

$$
R(y \cdot t)=R(y)
$$


Then $R$ preserves tiles and it passes to a retraction which we also denote by $R: X \rightarrow X^{0}$; it has the same property (4.6.1). Define $W: \bar{X} \rightarrow[0,1]$ by

$$
W(x)= \begin{cases}1 & \text { if } x \in X^{0}, \\ \sup _{\alpha \in \Delta_{Q}}\left\{1-r_{\alpha}^{Q}(x)\right\} & \text { if } x \in X^{Q} .\end{cases}
$$

We refer to $W$ as an exhaustion function because

$$
W^{-1}(0)=X^{0} \quad \text { and } \quad W^{-1}(1)=\bar{X}-X .
$$

The function $W$ is continuous (and piecewise analytic): If $\mathbf{P} \subset \mathbf{Q}$, write $\Delta_{P}=i\left(\Delta_{Q}\right) \amalg J$ as in (2.4.1). Let $\bar{P}=v_{Q}(P) \subset L_{Q}$ be the resulting parabolic subgroup of $L_{Q}$; then $\Delta_{\bar{P}}=J$. If $x \in \bar{X}^{P} \cap \bar{X}^{Q}$ then $\pi_{Q}(x) \in \bar{X}_{Q}^{P} \cap \bar{X}_{Q}^{0}$ so $r_{\alpha}^{P}(x)=r_{\alpha}^{\bar{P}}\left(\pi_{Q}(x)\right)=1$ for all $\alpha \in J$ as in $\S 3.4$. Hence

$$
\sup _{\alpha \in \Delta_{P}}\left\{1-r_{\alpha}^{P}(x)\right\}=\sup _{\alpha \in \Delta_{Q}}\left\{1-r_{\alpha}^{Q}(x)\right\}
$$

For each boundary stratum $X_{Q}$ the same constructions define a tile-preserving retraction

$$
R_{Q}: X_{Q} \rightarrow X_{Q}^{0}
$$

and an exhaustion function $W_{Q}: \bar{X}_{Q} \rightarrow[0,1]$ with $W_{Q}^{-1}(0)=X_{Q}^{0}$ and $W_{Q}^{-1}(1)=\bar{X}_{Q}-X_{Q}$. In fact, the stratum closure $\bar{X}_{Q}$ is tiled by the collection of intersections $X_{Q}^{P}=\bar{X}_{Q} \cap X^{P}$ for $\mathbf{P} \subseteq \mathbf{Q}$ and

$$
W_{Q}(x)= \begin{cases}1 & \text { if } x \in X_{Q}^{0}, \\ \sup _{\alpha \in J}\left\{1-r_{\alpha}^{P}(x)\right\} & \text { if } x \in X_{Q}^{P},\end{cases}
$$

where $\Delta_{P}=i\left(\Delta_{Q}\right) \amalg J$.

4.7. Remarks. We risk a certain amount of confusion by having defined $r_{\alpha}^{P}(x)$ so as to decrease as $x \rightarrow X_{P}$ whereas the root function $f_{\alpha}^{P}(x)$ increases as $x \rightarrow X_{P}$. Although Saper $[\mathrm{Sa}]$ actually constructs a tiling of the Borel-Serre compactification $\tilde{X}$ the same approach gives a tiling of the reductive Borel-Serre compactification $\bar{X}$. The collection $\left\{T\left(\bar{X}_{P}\right), \pi_{P}, r_{P}=\max _{\alpha \in \Delta_{P}}\left\{r_{\alpha}^{P}\right\}\right\}$ of tubular neighborhoods, tubular projections, and tubular distance functions are very much like a "system of control data" [Mat], [Gi], [GM3] for the stratified space $\bar{X}$, but there are several important differences. The functions $r_{P}$ are continuous and piecewise analytic but are not smooth. Whenever $\mathbf{Q} \subseteq \mathbf{P}$ we have $\pi_{Q} \pi_{P}=\pi_{Q}$ however we do not have $r_{Q} \pi_{P}=r_{Q}$. For this price we gain an especially strong form of "local triviality" for the stratification of $\bar{X}$ : the neighborhood $T\left(\bar{X}_{P}\right)$ is (homeomorphic to) a mapping cylinder neighborhood of the closure of the stratum $X_{P}$. In fact, it is possible to use the various geodesic actions to construct a (piecewise analytic) homeomorphism between $T\left(\bar{X}_{P}\right)$ and the (open) mapping cylinder of the projection $\pi_{P}: \partial T\left(\bar{X}_{P}\right) \rightarrow \bar{X}_{P}$. (The open mapping cylinder of a mapping $\pi: A \rightarrow B$ is the quotient $(A \times[0,1) \amalg B) / \sim$ under the relation $(a, 0) \sim \pi(a)$.) Analogous statements for other Satake compactifications (such as the Baily-Borel compactification) are false. 


\section{A little shrink}

5.1. As in $\S 2, \mathbf{G}$ denotes a connected linear reductive algebraic group defined over $\mathbb{Q}, D$ denotes the associated symmetric space, $K^{\prime}=A_{G} K\left(x_{0}\right)$ is the stabilizer in $G$ of a fixed basepoint $x_{0} \in D, \Gamma \subset \mathbf{G}(\mathbb{Q})$ is an arithmetic group and $X=\Gamma \backslash D$. In this section we construct a homeomorphism $\bar{X} \rightarrow \bar{X}$ which moves a neighborhood of the boundary towards the boundary. When composed with a Hecke correspondence, this will have the effect of chopping the fixed point set into pieces, each of which is contained in a single stratum of $\bar{X}$. The resulting behavior is much easier to analyze. This "shrink" homeomorphism may be considered to be a topological analog to Arthur's truncation procedure.

5.2. Let $\mathbf{Q} \in \mathscr{P}_{1}$ be a standard proper maximal rational parabolic subgroup of $\mathbf{G}$. Fix $t \in A_{Q}^{\prime}(>1)$ so $\alpha(t)>1$, where $\alpha \in \Delta_{Q}$ is the unique simple root. The geodesic action of $t$ on $D$ extends continuously to the neighborhood $D[Q](2.10 .2)$ of $D_{Q}$ in the reductive BorelSerre partial compactification $\bar{D}$ of $D$. This geodesic action even extends continuously to the neighborhood

$$
D\{Q\}=\bigcup_{\mathbf{P} \subset \mathbf{Q}} D[P]
$$

of the closure $\bar{D}_{Q}$, where the union is taken over all rational parabolic subgroups $\mathbf{P} \subset \mathbf{G}$ which are contained in $\mathbf{Q}$. (For if $\mathbf{P} \subset \mathbf{Q}$, let $i: A_{Q} \rightarrow A_{P}$ be the canonical inclusion. It follows from (2.4.4) (or [BS], Prop. 3.11) that the geodesic action of the image $i(t) \in A_{P}$ agrees with the geodesic action of $t \in A_{Q}$, so it extends continuously to $D[P]$.) We continue to denote this action by $x \mapsto x \cdot t$ for $x \in D\{Q\}$.

Now fix a sufficiently large $\Gamma$-invariant regular parameter $\mathbf{b} \in \mathscr{B}$ with its resulting tiling (4.1.1) and partial distance functions (4.2.2) satisfying Lemma 4.3. Let $T\left(\bar{D}_{Q}\right) \subset D\{Q\}$ denote the neighborhood of $\bar{D}_{Q}(4.2 .1)$ consisting of the union of all tiles which intersect $\bar{D}_{Q}$ nontrivially, and let $\bar{T}\left(\bar{D}_{Q}\right)$ denote its closure. The geodesic action by $t \in A_{Q}^{\prime}(>1)$ preserves $\bar{T}\left(\bar{D}_{Q}\right)$ since $\alpha(t)>1$.

Fix once and for all a smooth non-increasing function $\rho:[0,1] \rightarrow[0,1]$ with $\rho(r)=1 \Leftrightarrow r \leqq 1 / 2$ and with $\rho(r)=0 \Leftrightarrow r=1$.

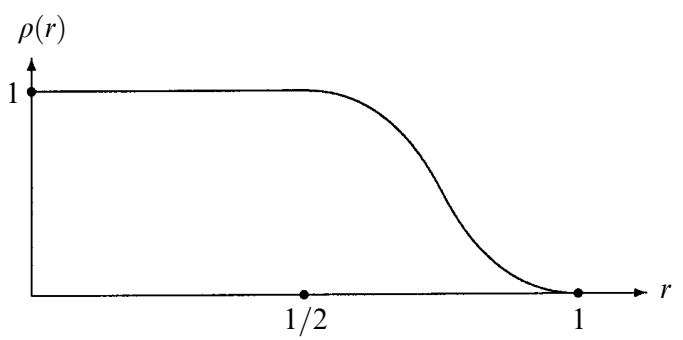

Figure 5. The function $\rho$.

Let $r_{\alpha}^{Q}: T\left(\bar{D}_{Q}\right) \rightarrow[0,1)$ be the partial distance function (4.2.2) which corresponds to the unique simple root $\alpha \in \Delta_{Q}$. For $t \in A_{Q}^{\prime}(>1)$ define $\operatorname{Sh}(Q, t): \bar{T}\left(\bar{D}_{Q}\right) \rightarrow \bar{T}\left(\bar{D}_{Q}\right)$ by 


$$
\operatorname{Sh}(Q, t)(x)=x \cdot t^{\rho\left(r_{\alpha}^{Q}(x)\right)} .
$$

Then by (3.2.2) and (4.3.1),

$$
\begin{aligned}
& r_{\alpha}^{Q}(\operatorname{Sh}(Q, t)(x))=r_{\alpha}^{Q}(x) \alpha(t)^{-\rho\left(r_{\alpha}^{Q}(x)\right)}, \\
& f_{\alpha}^{Q}(\operatorname{Sh}(Q, t)(x))=f_{\alpha}^{Q}(x) \alpha(t)^{\rho\left(r_{\alpha}^{Q}(x)\right)}
\end{aligned}
$$

for all $x \in \bar{T}\left(\bar{D}_{Q}\right)$. The quantity $\alpha(t)^{\rho\left(r_{\alpha}^{Q}(x)\right)}$ is bounded between 1 and $\alpha(t)$. It equals 1 if and only if $x \in \partial T\left(\bar{D}_{Q}\right)$, that is, if and only if $r_{\alpha}^{Q}(x)=1$.

If $\mathbf{Q}^{\prime}$ is another maximal rational parabolic subgroup of $\mathbf{G}$, let us write $\mathbf{Q}^{\prime} \sim \mathbf{Q}$ if $\mathbf{Q}^{\prime}$ is $\mathbf{G}(\mathbb{Q})$ conjugate to $\mathbf{Q}$. In this case, any choice $g \in \mathbf{G}(\mathbb{Q})$ of conjugating element induces the same isomorphism $\mathbf{S}_{\mathbf{Q}} \cong \mathbf{S}_{\mathbf{Q}^{\prime}}$ so we obtain a corresponding $t^{\prime} \in A_{Q^{\prime}}^{\prime}$ and a corresponding mapping $\operatorname{Sh}\left(Q^{\prime}, t^{\prime}\right): \bar{T}\left(\bar{D}_{Q^{\prime}}\right) \rightarrow \bar{T}\left(\bar{D}_{Q}\right)$. Define the shrink $S_{\mathbf{Q}}(t): \bar{D} \rightarrow \bar{D}$ corresponding to conjugates of the standard parabolic subgroup $\mathbf{Q}$ by

$$
S h_{\mathbf{Q}}(t)(x)= \begin{cases}\operatorname{Sh}\left(Q^{\prime}, t^{\prime}\right) x & \text { if } x \in \bar{T}\left(\bar{D}_{Q^{\prime}}\right) \text { for some } \mathbf{Q}^{\prime} \sim \mathbf{Q}, \\ x & \text { otherwise. }\end{cases}
$$

Then $S h_{\mathbf{Q}}(t)$ is well defined and continuous because $T\left(\bar{D}_{Q^{\prime}}\right) \cap T\left(\bar{D}_{Q}\right)=\emptyset$ whenever $\mathbf{Q}^{\prime} \sim \mathbf{Q}\left(\right.$ and $\left.\mathbf{Q}^{\prime} \neq \mathbf{Q}\right)$, cf. [Bo3], $\$ 11.17$ (iii). Moreover, if $\gamma \in \Gamma$ then

$$
S h_{\mathbf{Q}}(t)(\gamma x)=\gamma S h_{\mathbf{Q}}(t)(x)
$$

by Lemma 4.3. So (dividing by $\Gamma$ ), the homeomorphism $S h_{\mathbf{Q}}(t)$ passes to a homeomorphism which we denote in the same way, $S h_{\mathbf{Q}}(t): \bar{X} \rightarrow \bar{X}$.

5.3. Suppose $\mathbf{P} \subseteq \mathbf{Q}$ is a rational parabolic subgroup of $\mathbf{G}$; set $\Delta_{P}=i\left(\Delta_{Q}\right) \amalg J$ as in (2.4.1). It follows from (4.3.1) that for all $\beta \in J$ and for all $x \in \bar{T}\left(\bar{D}_{P}\right)$ we have

$$
r_{\beta}^{P} \operatorname{Sh}(Q, t)(x)=r_{\beta}^{P}(x)
$$

since $\beta(t)=1$ for any $\beta \in J$. Now suppose $\mathbf{Q}_{\mathbf{1}}, \mathbf{Q}_{\mathbf{2}}$ are two standard maximal rational parabolic subgroups of $\mathbf{G}$ whose intersection $\mathbf{P}=\mathbf{Q}_{\mathbf{1}} \cap \mathbf{Q}_{\mathbf{2}}$ is parabolic. Let $\alpha_{i} \in \Delta\left(Q_{i}\right)$ be the unique nonzero roots. Choose $t_{i} \in A_{i}^{\prime}=A_{Q_{i}}^{\prime}$ with $\alpha_{i}\left(t_{i}\right)>1$ and let

$$
S h_{i}=\operatorname{Sh}\left(Q_{i}, t_{i}\right): \bar{T}\left(\bar{D}_{Q_{i}}\right) \rightarrow \bar{T}\left(\bar{D}_{Q_{i}}\right)
$$

denote the resulting two shrinks. It follows by taking $\mathbf{P}=\mathbf{Q}_{1} \cap \mathbf{Q}_{2}$ in (5.3.1) that the mappings $S h_{1}$ and $S h_{2}$ commute on their common domain of definition,

$$
\bar{T}\left(\bar{D}_{P}\right)=\bar{T}\left(\bar{D}_{Q_{1}}\right) \cap \bar{T}\left(\bar{D}_{Q_{2}}\right)
$$

5.4. Let $\mathbf{P}_{\mathbf{0}} \subset \mathbf{G}$ be the standard minimal rational parabolic subgroup with $\mathbf{S}_{\mathbf{0}}^{\prime}=\mathbf{S}_{\mathbf{P}_{\mathbf{0}}} / \mathbf{S}_{\mathbf{G}}$ and with simple roots $\Delta=\left\{\alpha_{1}, \alpha_{2}, \ldots, \alpha_{r}\right\}$ numbered in any order. Each $j$ (with $1 \leqq j \leqq r$ ) corresponds to a standard maximal proper rational parabolic subgroup $\mathbf{Q}_{\mathbf{j}}$ with split torus $\mathbf{S}_{\mathbf{j}}^{\prime}=\mathbf{S}_{\mathbf{P}_{\mathbf{j}}} / \mathbf{S}_{\mathbf{G}}$ and identity component $A_{j}^{\prime}=\mathbf{S}_{\mathbf{j}}^{\prime}(\mathbb{R})^{0}$. Choose $\mathbf{t} \in A_{0}^{\prime}$ to be 
dominant and regular. In other words, with respect to the canonical complementary decomposition (cf. 2.4) $A_{0}^{\prime}=\prod_{j} A_{j}^{\prime}$ (with $\operatorname{ker}\left(\alpha_{j}\right)=\prod_{i \neq j} \mathbf{S}_{\mathbf{i}}^{\prime}$ ) we may write $\mathbf{t}=t_{1} t_{2} \ldots t_{r}$ where $t_{j} \in A_{j}^{\prime}$ and $\alpha_{j}\left(t_{j}\right)=\alpha_{j}(\mathbf{t})>1$. Define $\operatorname{Sh}(\mathbf{t}): \bar{D} \rightarrow \bar{D}$ to be the composition

$$
S h(\mathbf{t})=S h_{\mathbf{Q}_{1}}\left(t_{1}\right) \circ S h_{\mathbf{Q}_{2}}\left(t_{2}\right) \circ \cdots \circ S h_{\mathbf{Q}_{\mathbf{r}}}\left(t_{r}\right)
$$

where each $S h_{\mathbf{Q}_{\mathrm{j}}}\left(t_{j}\right): \bar{D} \rightarrow \bar{D}$ is the shrink defined above, corresponding to conjugates of the standard parabolic subgroup $\mathbf{Q}_{j}$.

5.5. Proposition. The mapping $\operatorname{Sh}(\mathbf{t}): \bar{D} \rightarrow \bar{D}$ is independent of the ordering $\Delta=\left\{\alpha_{1}, \alpha_{2}, \ldots, \alpha_{r}\right\}$ of the simple roots. It is a $\Gamma$-equivariant homeomorphism and passes to $a$ homeomorphism $\operatorname{Sh}(\mathbf{t}): \bar{X} \rightarrow \bar{X}$ with the following properties:

(1) It preserves the tiles and the strata, that is, for each rational parabolic subgroup $\mathbf{P} \subseteq \mathbf{G}$, we have $\operatorname{Sh}(\mathbf{t})\left(X^{P}\right)=X^{P}$ and $\operatorname{Sh}(\mathbf{t})\left(X_{P}\right)=X_{P}$.

(2) Within each tile, it is given by a geodesic action: for each $x \in X^{P}$ there exists $b=b_{x} \in A_{P}(\geqq 1)$ so that $\operatorname{Sh}(\mathbf{t})(x)=x \cdot b$. $x \in X^{P}$.

(3) It is the identity on each central tile $X_{P}^{0}$ and $\pi_{P}(\operatorname{Sh}(\mathbf{t})(x))=\pi_{P}(x) \in X_{P}^{0}$ for all

(4) It commutes with the geodesic projection, that is, for any rational parabolic subgroup $\mathbf{P} \subset \mathbf{G}$ and for each $x \in \bar{T}\left(\bar{X}_{P}\right)$ we have $\pi_{P}(\operatorname{Sh}(\mathbf{t})(x))=\operatorname{Sh}(\mathbf{t})\left(\pi_{P}(x)\right)$.

(5) It is (globally) homotopic to the identity.

(6) For any rational parabolic subgroup $\mathbf{P} \subset \mathbf{G}$ and for each $\alpha \in \Delta_{P}$ and for each $x \in \bar{T}\left(\bar{X}_{P}\right)$, by equation (5.2.2) we have:

$$
r_{\alpha}^{P}(\operatorname{Sh}(\mathbf{t})(x))=r_{\alpha}^{P}(x) \alpha_{0}(\mathbf{t})^{-\rho\left(r_{\alpha}^{P}(x)\right)}
$$

where $\alpha_{0} \in \Delta$ is the unique root which agrees with $\alpha$ after conjugation and restriction to $\mathbf{S}_{\mathbf{P}}$. If $x \in X^{P}$ is constrained to lie in the single tile $X^{P}$ then also

$$
f_{\alpha}^{P}(\operatorname{Sh}(\mathbf{t})(x))=f_{\alpha}^{P}(x) \alpha_{0}(\mathbf{t})^{\rho\left(r_{\alpha}^{P}(x)\right)} .
$$

We remark that the mapping $S h(\mathbf{t})$ depends on the choice of regular parameter (which determines the size of the tiles).

\section{Morphisms and Hecke correspondences}

6.1. As in $\$ 2, \mathbf{G}$ denotes a connected linear reductive algebraic group defined over $\mathbb{Q}, D$ denotes the associated symmetric space, and $K^{\prime}=A_{G} K\left(x_{0}\right)$ is the stabilizer in $G$ of a fixed basepoint $x_{0} \in D$. Let $\Gamma, \Gamma^{\prime} \subset \mathbf{G}(\mathbb{Q})$ be arithmetic subgroups and set $X=\Gamma \backslash D$ and $X^{\prime}=\Gamma^{\prime} \backslash D$. 
6.2. Definition. A mapping $f: X^{\prime} \rightarrow X$ is a morphism if there exists $g \in \mathbf{G}(\mathbb{Q})$ such that

(1) $g \Gamma^{\prime} g^{-1} \subset \Gamma$

(2) $\left[\Gamma: g \Gamma^{\prime} g^{-1}\right]<\infty$,

(3) $f\left(\Gamma^{\prime} x K^{\prime}\right)=\Gamma g x K^{\prime}$ for any $x \in G$.

The morphism $f$ is determined by the pair $\left(\Gamma^{\prime}, g\right)$ by $(3)$; it is well defined by (1). For any $\gamma \in \Gamma, \gamma^{\prime} \in \Gamma^{\prime}$ the pair $\left(\Gamma^{\prime}, \gamma g \gamma^{\prime}\right)$ determines the same morphism. If $\Gamma$ is torsion-free then $f$ is an unramified covering of degree $\left[\Gamma: g \Gamma^{\prime} g^{-1}\right]$ and it is locally an isometry with respect to the invariant Riemannian metrics on $X^{\prime}$ and $X$ induced from any $K$ invariant inner product on ${ }^{0} \mathfrak{p}(\S 2.1)$. Denote by $\operatorname{Mor}\left(X^{\prime}, X\right)$ the set of morphisms $X^{\prime} \rightarrow X$.

6.3. Lemma. Each morphism $f \in \operatorname{Mor}\left(X^{\prime}, X\right)$ admits unique continuous extensions $\tilde{f}: \tilde{X}^{\prime} \rightarrow \tilde{X}$ to the Borel-Serre compactification and $\bar{f}: \bar{X}^{\prime} \rightarrow \bar{X}$ to the reductive Borel-Serre compactification. The mappings $\tilde{f}$ and $\bar{f}$ are finite, and they restrict to morphisms on each boundary stratum. If $\bar{f}\left(X_{P}^{\prime}\right)=X_{Q}$, if $U^{\prime}$ and $U$ are $\Gamma^{\prime}$ and $\Gamma$-parabolic neighborhoods of $X_{P}^{\prime}$ and $X_{Q}$ in $\bar{X}^{\prime}$ and $\bar{X}$ respectively, then

$$
\bar{f}\left(\pi_{P}(x)\right)=\pi_{Q}(\bar{f}(x))
$$

for all $x \in U^{\prime} \cap \bar{f}^{-1}(U)$.

6.4. Proof. Suppose the morphism $f: X^{\prime} \rightarrow X$ is given by the pair $\left(\Gamma^{\prime}, g\right)$. Let $T_{g}: D \rightarrow D$ denote the action of $g$ on $D$. It moves the basepoint $x_{0}$ to a new basepoint $x_{1}=g x_{0}$ with stabilizer $K^{\prime}\left(x_{1}\right)={ }^{g} K^{\prime}=g K^{\prime} g^{-1}$. If $\mathbf{P}$ is a rational parabolic subgroup and if $\mathbf{Q}={ }^{\mathbf{g}} \mathbf{P}=g \mathbf{P} g^{-1}$ set $K_{P}^{\prime}\left(x_{0}\right)=K^{\prime} \cap P$ and $K_{Q}^{\prime}\left(x_{1}\right)=K^{\prime}\left(x_{1}\right) \cap Q$. Then $T_{g}$ may also be described as the mapping

$$
D=P / K_{P}^{\prime}\left(x_{0}\right) \rightarrow Q / K_{Q}^{\prime}\left(x_{1}\right)=D
$$

which is given by $x K_{P}^{\prime}\left(x_{0}\right) \mapsto g x g^{-1} K_{Q}^{\prime}\left(x_{1}\right)$ by (2.1.3). This intertwines the geodesic actions of $A_{P}^{\prime}$ and $A_{Q}^{\prime}$, that is,

$$
T_{g}(x \cdot a)=g x g^{-1} g i_{x_{0}}(a) g^{-1} K_{Q}^{\prime}\left(x_{1}\right)=g x g^{-1} i_{x_{1}}(\hat{a}) K_{Q}^{\prime}\left(x_{1}\right)=T_{g}(x) \cdot \hat{a}
$$

where $a \mapsto \hat{a}$ is the canonical identification $A_{P}^{\prime} \cong A_{Q}^{\prime}$ of $\S 2.3$. It follows that $T_{g}$ extends to a mapping $\tilde{T}_{g}: \tilde{D} \rightarrow \tilde{D}$ on the Borel-Serre partial compactification, which takes the boundary component $e_{P}=P / K_{P}^{\prime} A_{P}^{\prime}$ to $e_{Q}=Q / K_{Q}^{\prime} A_{Q}^{\prime}$ and satisfies

$$
\pi_{Q} \tilde{T}_{g}(x)=\tilde{T}_{g}\left(\pi_{P}(x)\right) .
$$

The mapping $\tilde{T}_{g}$ passes to a mapping $\tilde{f}: \Gamma^{\prime} \backslash \tilde{D} \rightarrow \Gamma \backslash \tilde{D}$ which is the desired extension. It maps $Y_{P}^{\prime}=\Gamma_{P}^{\prime} \backslash e_{P}$ to $Y_{Q}=\Gamma_{Q} \backslash e_{Q}$ by $\tilde{f}\left(\Gamma_{P}^{\prime} x K_{P}^{\prime}\left(x_{0}\right) A_{P}^{\prime}\right)=\Gamma_{Q} g x g^{-1} K_{Q}^{\prime}\left(x_{1}\right) A_{Q}^{\prime}$, which is a mapping of degree $\left[\Gamma_{Q}: g \Gamma_{P}^{\prime} g^{-1}\right]<\infty$. (Here, $\mathbf{Q}={ }^{g} \mathbf{P}$.) The extension $\tilde{f}$ may map several 
strata of $\tilde{X}^{\prime}$ to a single stratum of $\tilde{X}$ : let $\mathbf{Q}_{\mathbf{1}}, \mathbf{Q}_{\mathbf{2}}, \ldots, \mathbf{Q}_{\mathrm{m}}$ be a set of representatives for the ${ }^{g} \Gamma^{\prime}$-conjugacy classes of rational parabolic subgroups which are $\Gamma$-conjugate to $\mathbf{Q}$, and set $\mathbf{P}_{j}=g^{-1} \mathbf{Q}_{j} g$. Then $\tilde{f}$ maps each stratum $Y_{P_{j}}^{\prime} \subset \tilde{X}^{\prime}$ to the stratum $Y_{Q} \subset \tilde{X}$ by a morphism which may be described in a manner similar to (6.4.1). This shows that $\tilde{f}$ is finite and that its restriction to each boundary stratum is a morphism.

Similarly the mapping $\tilde{T}_{g}$ passes to a mapping $\bar{T}_{g}: \bar{D} \rightarrow \bar{D}$ on the reductive BorelSerre compactification of $D$, which further passes to a mapping $\bar{f}: \Gamma^{\prime} \backslash \bar{D} \rightarrow \Gamma \backslash \bar{D}$. Then $\bar{f}$ maps $X_{P}^{\prime}=\Gamma_{P}^{\prime} \backslash P / K_{P} A_{P} \mathscr{U}_{P}$ to $X_{Q}=\Gamma_{Q} \backslash Q / K_{Q} A_{Q} \mathscr{U}_{Q}$ by

$$
\bar{f}\left(\Gamma_{P}^{\prime} x K_{P}\left(x_{0}\right) A_{P} \mathscr{U}_{P}\right)=\Gamma_{Q} g x g^{-1} K_{Q}\left(x_{1}\right) A_{Q} \mathscr{U}_{Q}
$$

The degree of this mapping is not obviously finite because the intersection $\Gamma_{P}^{\prime} \cap \mathscr{U}_{P}$ is nontrivial. By (2.1.2) conjugation by $g$ takes $L_{P}\left(x_{0}\right)$ to $L_{Q}\left(x_{1}\right)$. Let $K_{L(P)}\left(x_{0}\right) A_{P}$ be the stabilizer in $L_{P}$ of the basepoint $\pi_{P}\left(x_{0}\right) \in D_{P}$ and let $K_{L(Q)}\left(x_{1}\right) A_{Q}$ be the stabilizer in $L_{Q}$ of the basepoint $\pi_{Q}\left(x_{1}\right) \in D_{Q}$. Set $\Gamma_{L(P)}^{\prime}=v_{P}\left(\Gamma_{P}^{\prime}\right) \subset L_{P}$ and $\Gamma_{L(Q)}=v_{Q}\left(\Gamma_{Q}\right) \subset L_{Q}$. Then $X_{P}^{\prime}=\Gamma_{L(P)}^{\prime} \backslash L_{P} / K_{L(P)}\left(x_{0}\right) A_{P}$ and $X_{Q}=\Gamma_{L(Q)} \backslash L_{Q} / K_{L(Q)}\left(x_{1}\right) A_{Q}$ with respect to which we may express $\bar{f}$ as follows:

$$
\bar{f}\left(\Gamma_{L(P)}^{\prime} x K_{L(P)}\left(x_{0}\right) A_{P}\right)=\Gamma_{L(Q)} g x g^{-1} K_{L(Q)}\left(x_{1}\right) A_{Q}
$$

which has degree $\left[\Gamma_{L(Q)}: g \Gamma_{L(P)}^{\prime} g^{-1}\right]<\infty$. As in the preceding paragraph, the mapping $\bar{f}$ will take each of the finitely many strata $X_{P_{j}}^{\prime}$ to the stratum $X_{Q}$ (for $1 \leqq j \leqq m$ ) by a similarly defined finite morphism.

6.5. Definition. A correspondence on $X=\Gamma \backslash G / K^{\prime}$ is an arithmetic subgroup $\Gamma^{\prime} \subset \mathbf{G}(\mathbb{Q})$ together with two morphisms $c_{1}, c_{2} \in \operatorname{Mor}(C, X)$, where $C=\Gamma^{\prime} \backslash D$. A point $x \in C$ is fixed if $c_{1}(x)=c_{2}(x)$. Two correspondences $\left(c_{1}, c_{2}\right): C \rightrightarrows X$ and $\left(c_{1}^{\prime}, c_{2}^{\prime}\right): C^{\prime} \rightrightarrows X$ are said to be isomorphic if there is an invertible morphism $\alpha: C \rightarrow C^{\prime}$ such that $c_{j}^{\prime} \circ \alpha=c_{j}$ (for $j=1,2$ ).

Each $g \in \mathbf{G}(\mathbb{Q})$ gives rise to a Hecke correspondence $C=C[g] \rightrightarrows X$ as follows: set $\Gamma[g]=\Gamma \cap g^{-1} \Gamma g, C=\Gamma[g] \backslash G / K^{\prime}$, and define

$$
\left(c_{1}, c_{2}\right)\left(\Gamma[g] x K^{\prime}\right)=\left(\Gamma x K^{\prime}, \Gamma g x K^{\prime}\right)
$$

Modifying $g$ by an element of $\mathbf{S}_{\mathbf{G}}(\mathbb{Q})$ does not change the Hecke correspondence. By Lemma 6.3 each correspondence $C \rightrightarrows X$ has a unique continuous extension $\bar{C} \rightrightarrows \bar{X}$ to the reductive Borel-Serre compactification, and an isomorphism $\alpha: C \rightarrow C^{\prime}$ of correspondences $C \rightrightarrows X, C^{\prime} \rightrightarrows X$ extends uniquely to an isomorphism $\bar{C} \rightarrow \bar{C}^{\prime}$ of the extended correspondences.

6.6. Lemma. Let $X=\Gamma \backslash D$ and let $g \in \mathbf{G}(\mathbb{Q})$. The isomorphism class of the resulting Hecke correspondence $\bar{C}=\bar{C}[g] \rightrightarrows \bar{X}$ depends only on the double coset $\Gamma g \Gamma \in \Gamma \backslash \mathbf{G}(\mathbb{Q}) / \Gamma$.

6.7. Proof. If suffices to verify the statement for the correspondence $C \rightrightarrows X$ since the extension to the reductive Borel-Serre compactification exists uniquely. Let $\gamma_{1}, \gamma_{2} \in \Gamma$ and 
let $g^{\prime}=\gamma_{1} g \gamma_{2}$ be another element in the same double coset $\Gamma g \Gamma$. Set $\Gamma\left[g^{\prime}\right]=\Gamma \cap g^{\prime-1} \Gamma g^{\prime}$, $C^{\prime}=\Gamma\left[g^{\prime}\right] \backslash G / K^{\prime}$, and define $\left(c_{1}^{\prime}, c_{2}^{\prime}\right): C^{\prime} \rightrightarrows X$ by

$$
c_{1}^{\prime}\left(\Gamma\left[g^{\prime}\right] x K^{\prime}\right)=\Gamma x K^{\prime}, \quad c_{2}^{\prime}\left(\Gamma\left[g^{\prime}\right] x K^{\prime}\right)=\Gamma g^{\prime} x K^{\prime}
$$

One verifies by direct calculation that the morphism $f: C^{\prime} \rightarrow C$ which is given by

$$
f\left(\Gamma\left[g^{\prime}\right] x K^{\prime}\right)=\Gamma[g] \gamma_{2} x K^{\prime}
$$

is a well-defined isomorphism of correspondences, with inverse given by $f^{-1}\left(\Gamma[g] x K^{\prime}\right)=\Gamma\left[g^{\prime}\right] \gamma_{2}^{-1} x K^{\prime}$.

6.8. Remark. It can be shown that the mapping $\left(c_{1}, c_{2}\right): C \rightarrow X \times X$ is generically one-to-one. In the event that every element of $g^{-1} \Gamma g \Gamma$ is neat, then this mapping is globally an embedding. The following proposition says every correspondence is a covering of a Hecke correspondence.

6.9. Proposition. Let $\Gamma^{\prime} \subset \mathbf{G}(\mathbb{Q})$ be an arithmetic subgroup, $C^{\prime}=\Gamma^{\prime} \backslash D$ and $\left(c_{1}^{\prime}, c_{2}^{\prime}\right): C^{\prime} \rightrightarrows X$ be a correspondence. Then there is a Hecke correspondence $\left(c_{1}, c_{2}\right): C[g] \rightrightarrows X$ and a subgroup $\Gamma^{\prime \prime} \subset \Gamma[g]$ such that the correspondence $C^{\prime} \rightrightarrows X$ is isomorphic to the correspondence

$$
C^{\prime \prime} \stackrel{h}{\rightarrow} C[g] \rightrightarrows X
$$

where $C^{\prime \prime}=\Gamma^{\prime \prime} \backslash G / K$ and $h\left(\Gamma^{\prime \prime} x K\right)=\Gamma[g] x K$.

6.10. Proof. Suppose $\quad c_{1}^{\prime}\left(\Gamma^{\prime} x K^{\prime}\right)=\Gamma g_{1} x K^{\prime} \quad$ and $\quad c_{2}^{\prime}\left(\Gamma^{\prime} x K^{\prime}\right)=\Gamma g_{2} x K^{\prime} \quad$ where $g_{j} \Gamma^{\prime} g_{j}^{-1} \subset \Gamma$ are subgroups of finite index. Then $g=g_{2} g_{1}^{-1}$ determines a Hecke correspondence $C[g]=\Gamma[g] \backslash D \rightrightarrows X$. Define $\Gamma^{\prime \prime}=g_{1} \Gamma^{\prime} g_{1}^{-1}$ and $C^{\prime \prime}=\Gamma^{\prime \prime} \backslash D$. Since $\Gamma^{\prime \prime} \subset \Gamma[g]$ we obtain a correspondence in "standard form",

$$
C^{\prime \prime} \stackrel{h}{\rightarrow} C[g] \rightrightarrows X
$$

with $h\left(\Gamma^{\prime \prime} x K^{\prime}\right)=\Gamma[g] x K^{\prime}$. Define $f: C^{\prime} \rightarrow C^{\prime \prime}$ by $f\left(\Gamma^{\prime} x K^{\prime}\right)=\Gamma^{\prime \prime} g_{1} x K^{\prime}$. Then $f$ is well defined, and it is easily seen to be an isomorphism of correspondences.

6.11. Narrow tilings. Let $\left(c_{1}, c_{2}\right): \bar{C} \rightrightarrows \bar{X}$ be a Hecke correspondence defined by some element $g \in \mathbf{G}(\mathbb{Q})$, so $C=\Gamma^{\prime} \backslash D$ with $\Gamma^{\prime}=\Gamma[g]=\Gamma \cap g^{-1} \Gamma g$. Let $b \in \mathscr{B}$ be a sufficiently large $\Gamma$-invariant regular parameter. Then it is also $\Gamma^{\prime}$-invariant, it gives rise to tilings $\left\{C^{Q}\right\}$ of $\bar{C}$ and $\left\{X^{Q}\right\}$ of $\bar{X}$, and the mapping $c_{1}: \bar{C} \rightarrow \bar{X}$ takes tiles to tiles (although the same cannot necessarily be said of $c_{2}$ ). Let us say this tiling is narrow with respect to the Hecke correspondence if, for every stratum $C_{Q}$ of $\bar{C}$, the following holds:

$$
c_{1}\left(\bar{T}\left(\bar{C}_{Q}\right)\right) \cap c_{2}\left(\bar{T}\left(\bar{C}_{Q}\right)\right) \neq \emptyset \Leftrightarrow c_{1}\left(C_{Q}\right)=c_{2}\left(C_{Q}\right)
$$

and if, in this case, $c_{1}\left(\bar{T}\left(\bar{C}_{Q}\right)\right) \cup c_{2}\left(\bar{T}\left(\bar{C}_{Q}\right)\right)$ is a $\Gamma$-parabolic neighborhood of $\bar{X}_{Q}$ in $\bar{X}$.

6.12. Proposition. Fix a Hecke correspondence $\bar{C} \rightrightarrows \bar{X}$. If the $\Gamma$-invariant regular 
parameter $b \in \mathscr{B}$ is chosen sufficiently large then the resulting tiling $\left\{C^{Q}\right\}$ of $\bar{C}$ is narrow for that Hecke correspondence.

6.13. Proof. Let $C_{Q}$ be a stratum of $\bar{C}$ and suppose $c_{1}\left(C_{Q}\right)=X_{P}$ and $c_{2}\left(C_{Q}\right)=X_{P^{\prime}}$. Then $\mathbf{Q}$ is $\Gamma$-conjugate to $\mathbf{P}$ while $g \mathbf{Q} g^{-1}$ is $\Gamma$-conjugate to $\mathbf{P}^{\prime}$ (cf. Lemma 7.4). In particular, $\mathbf{P}$ and $\mathbf{P}^{\prime}$ are $\mathbf{G}(\mathbb{Q})$-conjugate, which implies that either $X_{P}=X_{P^{\prime}}$ or $\bar{X}_{P} \cap \bar{X}_{P^{\prime}}=\emptyset$ (2.10.4). In the latter case there exist neighborhoods $U$ of $\bar{X}_{P}$ and $U^{\prime}$ of $\bar{X}_{P^{\prime}}$ which do not intersect. Choose the tiling parameter so large that $\bar{T}\left(\overline{C_{Q}}\right) \subset c_{1}^{-1}(U) \cap c_{2}^{-1}\left(U^{\prime}\right)$. Since there are finitely many strata $C_{Q}$ in $\bar{C}$, this amounts to finitely many conditions on the tiling parameter. On the other hand, if $c_{1}\left(C_{Q}\right)=c_{2}\left(C_{Q}\right)=X_{P}$, then we may take $\mathbf{P}=\mathbf{Q}$. Choose any parabolic neighborhood $U \subset \bar{X}$ of $\bar{X}_{Q}$ and then choose the tiling so small that $\bar{T}\left(\bar{C}_{Q}\right) \subset c_{1}^{-1}(U) \cap c_{2}^{-1}(U)$. This guarantees that $c_{1}\left(\bar{T}\left(\bar{C}_{Q}\right)\right) \cup c_{2}\left(\bar{T}\left(\bar{C}_{Q}\right)\right)$ is a $\Gamma$-parabolic set in $\bar{X}$.

\section{Restriction to the boundary}

7.1. Parabolic Hecke correspondence. As in $\S 2$, G denotes a connected linear reductive algebraic group defined over $\mathbb{Q}, D$ denotes the associated symmetric space, $K^{\prime}=A_{G} K\left(x_{0}\right)$ is the stabilizer in $G$ of a fixed basepoint $x_{0} \in D, \Gamma \subset \mathbf{G}(\mathbb{Q})$ is an arithmetic group and $X=\Gamma \backslash D$. Fix a rational parabolic subgroup $\mathbf{P} \subset \mathbf{G}$ and let $X_{P}=\Gamma_{P} \backslash D_{P} \subset \bar{X}$ be the corresponding stratum in the reductive Borel-Serre compactification of $X$. Each $y \in \mathbf{P}(\mathbb{Q})$ determines a correspondence on a $\mathbf{P}(\mathbb{Q})$-invariant neighborhood of $X_{P}$ which we now describe. Set $\Gamma_{P}^{\prime}=\Gamma_{P}[y]=\Gamma_{P} \cap y^{-1} \Gamma_{P} y$. Define the parabolic Hecke correspondence

$$
\left(c_{1}, c_{2}\right): \Gamma_{P}^{\prime} \backslash D[P] \rightrightarrows \Gamma_{P} \backslash D[P]
$$

determined by $y \in \mathbf{P}(\mathbb{Q})$ to be the unique continuous extension of the correspondence $\Gamma_{P}^{\prime} \backslash D \rightrightarrows \Gamma_{P} \backslash D$ which is given by

$$
\Gamma_{P}^{\prime} x K_{P}^{\prime} \mapsto\left(\Gamma_{P} x K_{P}^{\prime}, \Gamma_{P} y x K_{P}^{\prime}\right)
$$

where we identify $D=P / K_{P}^{\prime}$. It follows from (6.4.2) (by taking $\mathbf{P}=\mathbf{Q}$ ) that this correspondence commutes with the geodesic action of $A_{P}^{\prime}$, that is,

$$
c_{i}(x \cdot a)=c_{i}(x) \cdot a
$$

(for $i=1,2$ ) for any $x \in \Gamma_{P}^{\prime} \backslash D[P]$ and for any $a \in A_{P}^{\prime}$. Therefore the parabolic Hecke correspondence preserves the corner structure near $C_{P}$, that is, if $\mathbf{Q} \supset \mathbf{P}$ is a rational parabolic subgroup then each mapping $c_{i}$ takes the stratum $\Gamma_{P}^{\prime} \backslash D_{Q} \subset \Gamma_{P}^{\prime} \backslash D[P]$ to the stratum $\Gamma_{P} \backslash D_{Q} \subset \Gamma_{P} \backslash D[P]$.

There is also an associated (global) correspondence, $\bar{C}=\Gamma^{\prime} \backslash \bar{D} \rightrightarrows \bar{X}=\Gamma \backslash \bar{D}$ (where $\left.\Gamma^{\prime}=\Gamma \cap y^{-1} \Gamma y\right)$. If $V \subset D[P] \subset \bar{D}$ is a $\Gamma$-parabolic neighborhood of $D_{P}$ then it is also a $\Gamma^{\prime}$ parabolic neighborhood of $D_{P}$, as is $y^{-1} \cdot V$. Hence $V \cap y^{-1} V$ is also a $\Gamma^{\prime}$-parabolic neighborhood of $D_{P}$. It follows that, if $U \subset \Gamma_{P} \backslash D[P] \subset \Gamma_{P} \backslash \bar{D}$ is a $\Gamma$-parabolic neighborhood of $X_{P}$ then $U^{\prime}=c_{1}^{-1}(U) \cap c_{2}^{-1}(U) \subset \Gamma_{P}^{\prime} \backslash \bar{D}$ is a $\Gamma^{\prime}$-parabolic neighborhood of $C_{P}=\Gamma_{P}^{\prime} \backslash D_{P}$. We will say that any correspondence isomorphic to such a $U^{\prime} \rightrightarrows U$ is modeled on the parabolic Hecke correspondence (7.1.1). 


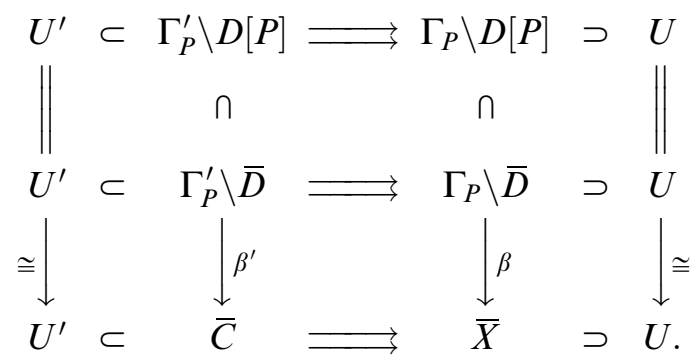

7.2. Now suppose $g \in \mathbf{G}(\mathbb{Q})$ gives rise to the Hecke correspondence $C=C[g] \rightrightarrows X$ with its canonical extension $\left(c_{1}, c_{2}\right): \bar{C} \rightrightarrows \bar{X}$, where $C=\Gamma[g] \backslash D$ as in $\S 6.5 .1$. If $\mathbf{P}$ is a rational parabolic subgroup of $\mathbf{G}$ and if $X_{P}$ denotes the corresponding RBS stratum, then we may consider the part $c_{1}^{-1}\left(X_{P}\right)$ of $\bar{C}$ which lies over this stratum. It will consist of several RBS boundary strata $C_{Q}$ of $C$. Some of these boundary strata may be mapped back to $X_{P}$ by the mapping $c_{2}$. In this case, we shall say that the Hecke correspondence $\bar{C}$ has a restriction to $X_{P}$ consisting of the union of those boundary strata $C_{Q}$ such that $\left(c_{1}, c_{2}\right) \mid C_{Q}: C_{Q} \rightrightarrows X_{Q}$.

7.3. Proposition. Let $\Gamma \subset \mathbf{G}(\mathbb{Q})$ be a neat arithmetic group. Let

$$
\left(c_{1}, c_{2}\right): C=C[g] \rightrightarrows X
$$

be the Hecke correspondence which is determined by an element $g \in \mathbf{G}(\mathbb{Q})$. Let $\mathbf{P}$ be a rational parabolic subgroup of $\mathbf{G}$, with corresponding boundary stratum $X_{P} \subset \bar{X}$. Decompose the intersection $\Gamma g \Gamma \cap P$ into a union of $\Gamma_{P}$-double cosets,

$$
\Gamma g \Gamma \cap P=\coprod_{j=1}^{m} \Gamma_{P} g_{j} \Gamma_{P}
$$

with $g_{j} \in \mathbf{P}(\mathbb{Q})$. Then $m<\infty$ and, over a sufficiently small parabolic neighborhood of $X_{P}$, the Hecke correspondence $\bar{C} \rightrightarrows \bar{X}$ breaks into a disjoint union of $m$ correspondences which are given by $g_{j}$ and which are modeled on the parabolic Hecke correspondences

$$
\Gamma_{P}\left[g_{j}\right] \backslash D[P] \rightrightarrows \Gamma_{P} \backslash D[P]
$$

for $j=1,2, \ldots, m$, where $\Gamma_{P}\left[g_{j}\right]=\Gamma \cap g_{j}^{-1} \Gamma g_{j} \cap P$.

(A similar procedure is described in the adelic setting in $[\mathrm{H}]$.) The proof will take the rest of \$7. First we establish a one-to-one correspondence between the components of the restriction of the Hecke correspondence to $X_{P}$ and the double cosets which appear in (7.3.1). Let $\Gamma_{P} y \Gamma_{P} \subset \Gamma g \Gamma \cap P$ be a double coset from (7.3.1). Write $y=\gamma_{2} g \gamma_{1}$ for some $\gamma_{1}, \gamma_{2} \in \Gamma$. Set $\Gamma[g]=\Gamma \cap g^{-1} \Gamma g$. Define

$$
\Xi\left(\Gamma_{P} y \Gamma_{P}\right)=\gamma_{1} \mathbf{P} \gamma_{1}^{-1}=g^{-1} \gamma_{2}^{-1} \mathbf{P} \gamma_{2} g .
$$

7.4. Lemma. The mapping $\Xi$ gives a well defined one-to-one correspondence between

(a) double cosets $\Gamma_{P} y \Gamma_{P} \subset \Gamma g \Gamma \cap P$, 
(b) $\Gamma[g]$-conjugacy classes of rational parabolic subgroups $\mathbf{Q}=\Xi\left(\Gamma_{P} y \Gamma_{P}\right) \subset \mathbf{G}$ such that

(i) $\mathbf{Q}$ is $\Gamma$-conjugate to $\mathbf{P}$ and

(ii) $g \mathbf{Q} g^{-1}$ is $\Gamma$-conjugate to $\mathbf{P}$,

(c) boundary strata $C_{Q} \subset \bar{C}$ such that $\left(c_{1}, c_{2}\right): C_{Q} \rightrightarrows X_{P}$.

In particular, this set is finite.

7.5. Proof of Lemma 7.4. First compare the sets (b) and (c). The boundary strata in $\bar{C}^{\prime}$ are in one-to-one correspondence with $\Gamma[g]$-conjugacy classes of rational parabolic subgroups of $\mathbf{G}$, while the boundary strata in $\bar{X}$ are in one-to-one correspondence with $\Gamma$ conjugacy classes of rational parabolic subgroups. Condition (i) is equivalent to the statement that $c_{1}$ maps $C_{Q}$ to $X_{P}$ while condition (ii) is equivalent to the statement that $c_{2}$ maps $C_{Q}$ to $X_{P}$.

Now verify that the mapping $\Xi$ is well defined, i.e. that the $\Gamma[g]$-conjugacy class of $\Xi\left(\Gamma_{P} g_{j} \Gamma_{P}\right)=\gamma_{1} \mathbf{P} \gamma_{1}^{-1}$ is independent of the choices. Let $y^{\prime}=\gamma_{2}^{\prime} g \gamma_{1}^{\prime} \in \Gamma_{P} g_{j} \Gamma_{P}$ be another element in the same double coset, and set $\mathbf{Q}^{\prime}=\gamma_{1}^{\prime} \mathbf{P}\left(\gamma_{1}^{\prime}\right)^{-1}$. Since $y^{\prime} \in \Gamma_{P} y \Gamma_{P}$, there exists $\gamma_{a}, \gamma_{b} \in \Gamma_{P}$ such that $y^{\prime}=\gamma_{a} y \gamma_{b}$. So $y^{\prime}=\gamma_{2}^{\prime} g \gamma_{1}^{\prime}=\gamma_{a} \gamma_{2} g \gamma_{1} \gamma_{b}$, which gives

$$
h:=g^{-1} \gamma_{2}^{\prime-1} \gamma_{a} \gamma_{2} g=\gamma_{1} \gamma_{b} \gamma_{1}^{\prime-1} \in g^{-1} \Gamma g \cap \Gamma=\Gamma[g]
$$

Then,

$$
h^{-1} \mathbf{Q} h=\left(\gamma_{1} \gamma_{b} \gamma_{1}^{\prime-1}\right)^{-1}\left(\gamma_{1} \mathbf{P} \gamma_{1}^{-1}\right)\left(\gamma_{1} \gamma_{b} \gamma_{1}^{\prime-1}\right)=\mathbf{Q}^{\prime}
$$

which verifies that $\mathbf{Q}$ and $\mathbf{Q}^{\prime}$ are $\Gamma[g]$ conjugate.

Next we show that $\Xi$ is surjective. Suppose that $\mathbf{Q}$ and $g \mathbf{Q} g^{-1}$ are both $\Gamma$-conjugate to P. Say, $Q=\gamma_{1} \mathbf{P} \gamma_{1}^{-1}$ and $g \mathbf{Q} g^{-1}=\gamma_{2}^{-1} \mathbf{P} \gamma_{2}$ for some $\gamma_{1}, \gamma_{2} \in \Gamma$. Then

$$
\gamma_{2} g \gamma_{1} \mathbf{P} \gamma_{1}^{-1} g^{-1} \gamma_{2}^{-1}=\mathbf{P}
$$

so the element $y=\gamma_{2} g \gamma_{1} \in \mathbf{P}(\mathbb{Q}) \cap \Gamma g \Gamma$ (since a parabolic subgroup is its own normalizer) and $\Xi\left(\Gamma_{P} y \Gamma_{P}\right)=\mathbf{Q}$.

Finally we show that $\Xi$ is injective. Suppose $y, y^{\prime} \in \Gamma g \Gamma \cap P$, say $y=\gamma_{2} g \gamma_{1}$ and $y^{\prime}=\gamma_{2}^{\prime} g \gamma_{1}^{\prime}$. Set

$$
\begin{aligned}
\mathbf{Q} & =\gamma_{1} \mathbf{P} \gamma_{1}^{-1}=g^{-1} \gamma_{2}^{-1} \mathbf{P} \gamma_{2} g, \\
\mathbf{Q}^{\prime} & =\gamma_{1}^{\prime} \mathbf{P}\left(\gamma_{1}^{\prime}\right)^{-1}=g^{-1}\left(\gamma_{2}^{\prime}\right)^{-1} \mathbf{P} \gamma_{2} g .
\end{aligned}
$$

Suppose $\mathbf{Q}$ and $\mathbf{Q}^{\prime}$ are $\Gamma[g]$-conjugate, say $\mathbf{Q}=\gamma \mathbf{Q}^{\prime} \gamma^{-1}$ for some $\gamma \in \Gamma \cap g^{-1} \Gamma g$. Comparing these two relations gives 


$$
\begin{aligned}
& \mathbf{Q}=\gamma_{1} \mathbf{P} \gamma_{1}^{-1}=\gamma \gamma_{1}^{\prime} \mathbf{P}\left(\gamma_{1}^{\prime}\right)^{-1} \gamma^{-1}, \\
& \mathbf{Q}=g^{-1} \gamma_{2}^{-1} \mathbf{P} \gamma_{2} g=\gamma g^{-1}\left(\gamma_{2}^{\prime}\right)^{-1} \mathbf{P} \gamma_{2}^{\prime} g \gamma^{-1},
\end{aligned}
$$

from which it follows that $h_{1}=\left(\gamma_{1}^{\prime}\right)^{-1} \gamma^{-1} \gamma_{1} \in P$ and $h_{2}=\gamma_{2} g \gamma g^{-1}\left(\gamma_{2}^{\prime}\right)^{-1} \in P$. Moreover, $h_{1}, h_{2} \in \Gamma$. But $h_{2} y^{\prime} h_{1}=y$ hence $\Gamma_{P} y^{\prime} \Gamma_{P}=\Gamma_{P} y \Gamma_{P}$ as claimed.

7.6. Proof of Proposition 7.3. By Lemma 7.4, the restriction of the Hecke correspondence $\bar{C}=\bar{C}[g] \rightrightarrows \bar{X}$ to the stratum $X_{P}$ breaks into a union of $m$ correspondences, indexed by the elements $g_{1}, g_{2}, \ldots, g_{m}$. Fix $j$ (with $1 \leqq j \leqq m$ ) and set $\Gamma\left[g_{j}\right]=\Gamma \cap g_{j}^{-1} \Gamma g_{j}$ and $\Gamma_{P}\left[g_{j}\right]=\Gamma\left[g_{j}\right] \cap P$. The following commutative diagram of correspondences provides an explicit isomorphism between the parabolic Hecke correspondence given by $g_{j}$ with the corresponding piece of the Hecke correspondence given by $g$ :

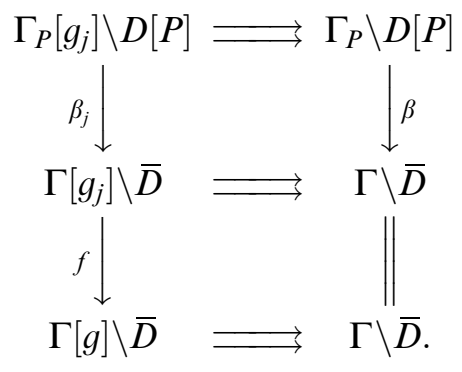

The first line is the parabolic Hecke correspondence (7.1.1) defined by $g_{j}$, i.e., it is the continuous extension of the mapping $\Gamma_{P}\left[g_{j}\right] x K_{P}^{\prime} \mapsto\left(\Gamma_{P} x K_{P}^{\prime}, \Gamma_{P} g_{j} x K_{P}^{\prime}\right)$. The second line is the Hecke correspondence (6.5.1) defined by $g_{j}$, i.e. it is the continuous extension of $\Gamma\left[g_{j}\right] x K^{\prime} \mapsto\left(\Gamma x K^{\prime}, \Gamma g x K^{\prime}\right)$. The vertical mapping $\beta$ (resp. $\beta_{j}$ ) is described in $\S 3.1$; it is a homeomorphism over any $\Gamma$-parabolic neighborhood of $X_{P}$ (resp. over any $\Gamma\left[g_{j}\right]$-parabolic neighborhood of $\left.\Gamma_{P}\left[g_{j}\right] \backslash D_{P}\right)$. The top square of this diagram commutes by direct computation. The third line is the given Hecke correspondence (6.5.1). The vertical mapping $f$ is the isomorphism of Hecke correspondences given in Lemma 6.6 and equation (6.7.1). In other words, if $g_{j}=\gamma_{1} g \gamma_{2}$ then $f\left(\Gamma\left[g_{j}\right] x K^{\prime}\right)=\Gamma[g] \gamma_{2} x K^{\prime}$. The bottom square also commutes. This completes the construction of an explicit isomorphism with the parabolic Hecke correspondence, and hence of the proof of Proposition 7.3.

\section{Counting the fixed points}

8.1. As in $\S 2, \mathbf{G}$ denotes a connected linear reductive algebraic group defined over $\mathbb{Q}, D$ denotes the associated symmetric space, $K^{\prime}=A_{G} K\left(x_{0}\right)$ is the stabilizer in $G$ of a fixed basepoint $x_{0} \in D, \Gamma \subset \mathbf{G}(\mathbb{Q})$ is an arithmetic group and $X=\Gamma \backslash D$. Through this section we assume that $\Gamma$ is neat. Fix a rational parabolic subgroup $\mathbf{P} \subseteq \mathbf{G}$, set $\Gamma_{P}=\Gamma \cap P$, $\Gamma_{L}=v_{P}\left(\Gamma_{P}\right) \subset \mathbf{L}_{\mathbf{P}}(\mathbb{Q})$ and denote by $X_{P}=\Gamma_{P} \backslash D_{P}=\Gamma_{L} \backslash D_{P}$ the corresponding stratum in the reductive Borel-Serre compactification $\bar{X}$. An element $y \in \mathbf{P}(\mathbb{Q})$ gives rise to a parabolic Hecke correspondence $\left(c_{1}, c_{2}\right): \Gamma_{P}^{\prime} \backslash D[P] \rightrightarrows \Gamma_{P} \backslash D[P]$ where $\Gamma_{P}^{\prime}=\Gamma_{P}[y]=\Gamma_{P} \cap y^{-1} \Gamma_{P} y$. Let $\Gamma_{L}^{\prime}=v_{P}\left(\Gamma_{P}^{\prime}\right)$. The restriction $C_{P} \rightrightarrows X_{P}$ of this parabolic correspondence to the boundary stratum $C_{P}=\Gamma_{L}^{\prime} \backslash D_{P}$ is given by

$$
\left(c_{1}, c_{2}\right)\left(\Gamma_{L}^{\prime} x K_{P} A_{P}\right)=\left(\Gamma_{L} x K_{P} A_{P}, \Gamma_{L} \bar{y} x K_{P} A_{P}\right)
$$

where $\bar{y}=v_{P}(y)$. 
8.2. Characteristic element. Let us suppose that $w \in C_{P}$ is a fixed point of the parabolic Hecke correspondence, that is, $c_{1}(w)=c_{2}(w)$. Choose any lift $\tilde{w} \in D_{P}$ of $w$ and write $\tilde{w}=z K_{P} A_{P} \in L_{P} / K_{P} A_{P}$. Since the point $w=\Gamma_{L}^{\prime} z K_{P} A_{P}$ is fixed, we have

$$
\Gamma_{L} z K_{P} A_{P}=\Gamma_{L} \bar{y} z K_{P} A_{P}
$$

Since $\Gamma$ is neat, there exists a unique $\gamma \in \Gamma_{L}$ such that the element $e=\gamma \bar{y}$ fixes the point $\tilde{w} \in D_{P}$, that is,

$$
e z K_{P} A_{P}=\gamma \bar{y} z K_{P} A_{P}=z K_{P} A_{P} \in D_{P}
$$

8.3. Definition. The element $e=\gamma \bar{y} \in \mathbf{L}_{\mathbf{P}}(\mathbb{Q})$ is called a characteristic element for the fixed point $w$, or the characteristic element corresponding to the lift $\tilde{w}$ of $w$.

Denote by $F_{P}(e) \subset C_{P}$ the set of fixed points in $C_{P}$ for which $e$ is a characteristic element. We refer to $F_{P}(e)$ as a fixed point constituent; it may consist of several connected components. Let $D_{P}^{e}$ denote the fixed points of the mapping $T_{e}: D_{P} \rightarrow D_{P}$ which is given by translation by $e$. Then $F_{P}(e)$ is the image of $D_{P}^{e}$ under the projection $D_{P} \rightarrow C_{P}$. Let $L_{e} \subset L_{P}$ denote the centralizer of $e$ in $L_{P}$. We say an element of $L_{P}$ is elliptic (or is elliptic modulo $\left.A_{P}\right)$ if it is $\mathbf{L}_{\mathbf{P}}(\mathbb{R})$-conjugate to an element of $K_{P} A_{P}$.

8.4. Proposition. Let $e=a_{e} m_{e} \in A_{P} M_{P}$ be the characteristic element corresponding to a lift $\tilde{w} \in D_{P}$ of the fixed point $w \in C_{P}$. Then the following statements hold.

(1) The characteristic element $e \in L_{P}$ is semisimple and is elliptic (modulo $A_{P}$ ). The group $L_{e}$ is reductive, algebraic, and defined over $\mathbb{Q}$. The torus factors $a_{y}=a_{e} \in A_{P}$ are equal (§2.2). The fixed point constituent $F_{P}(e)$ is a smooth submanifold of $C_{P}$.

(2) If $y \in P$ is changed by multiplication by an element $u \in \mathscr{U}_{P}$, or if $\gamma \in \Gamma_{L}$ is replaced by another element of $\Gamma_{L}$ which also satisfies (8.2.2), or if a different representative $z^{\prime} \in L_{P}$ of $\tilde{w} \in D_{P}$ is chosen, then the characteristic element $e \in L_{P}$ does not change.

(3) If a different lift $\tilde{w}^{\prime} \in D_{P}$ of $w$ is chosen, or if $y$ is changed within its double coset $\Gamma_{P} y \Gamma_{P}$ then e changes at most by $\Gamma_{L}$-conjugacy.

(4) The characteristic element e is a rigid invariant of the fixed point set: if $w_{t} \in C_{P}$ is a one parameter family of fixed points (with $t \in[0,1]$ ) and if $z_{t} \in D_{P}$ is a lift to a one parameter family of points in $D_{P}$ then the resulting characteristic elements $e_{t}$ do not vary with $t$.

(5) The group $L_{e}$ acts transitively on $D_{P}^{e}$. Set $\Gamma_{e}=\Gamma_{L} \cap L_{e}, \Gamma_{e}^{\prime}=\Gamma_{L}^{\prime} \cap L_{e}$, and $K_{e}^{\prime}=L_{e} \cap\left(z\left(K_{P} A_{P}\right) z^{-1}\right)$. Then $K_{e}^{\prime}$ contains a maximal compact subgroup of $L_{e}$. The action of $L_{e}$ on $D_{P}^{e}$ induces diffeomorphisms

$$
F_{P}(e) \cong \Gamma_{e}^{\prime} \backslash L_{e} / K_{e}^{\prime} \quad \text { and } \quad c_{i}\left(F_{P}(e)\right) \cong \Gamma_{e} \backslash L_{e} / K_{e}^{\prime}
$$

The projection $F_{P}(e) \rightarrow c_{i}\left(F_{P}(e)\right)$ is a covering of degree

$$
d_{e}=\left[\Gamma_{e}: \Gamma_{e}^{\prime}\right]=\left[\Gamma_{L} \cap \bar{y}^{-1} \Gamma_{L} \bar{y}: v_{P}\left(\Gamma_{P} \cap y^{-1} \Gamma_{P} y\right)\right]
$$


(6) Conversely, let $\mathscr{C} \subset \Gamma_{L} \bar{y} \Gamma_{L}$ be any $\Gamma_{L}$-conjugacy class which is elliptic (modulo $A_{P}$ ). Then $\mathscr{C} \cap \Gamma_{L} \bar{y}$ consists of a single element $e^{\prime}$, and there exists a fixed point $w^{\prime} \in C_{P}$ for which $e^{\prime}$ is a characteristic element. In particular, $F_{P}\left(e^{\prime}\right) \neq \emptyset$.

Hence the constituents of the fixed point set in $C_{P}$ are in one-to-one correspondence with $\Gamma_{L}$-conjugacy classes of elliptic (modulo $A_{P}$ ) elements $e \in \Gamma_{L} \bar{y} \Gamma_{L}$.

8.5. Proof. By (8.2.2), $e=\gamma \bar{y} \in z K_{P} A_{P} z^{-1}$ which is compact modulo $A_{P}$, so $e$ is also semisimple. The elements $e$ and $\bar{y}$ are in the same $\Gamma_{L}$-double coset so they have the same torus component $a_{e}=a_{y} \in A_{P}$. Since $\Gamma$ is neat, the group $\Gamma_{e}$ acts freely on $D_{P}^{e}$. This proves (1). Next, consider (2) and suppose different choices $y^{\prime}=y u, \gamma^{\prime} \in \Gamma_{L}$, and $z^{\prime} \in L_{P}$ were made, with $u \in \mathscr{U}_{P}$, with $\tilde{w}=z K_{P} A_{P}=z^{\prime} K_{P} A_{P} \in D_{P}$ and, as in (8.2.2), $z^{\prime} K_{P} A_{P}=\gamma^{\prime} \bar{y}^{\prime} z^{\prime} K_{P} A_{P}$ (where $\left.\bar{y}^{\prime}=v_{P}\left(y^{\prime}\right)\right)$. Then $\bar{y}^{\prime}=\bar{y}$ and

$$
\gamma \bar{y} z K_{P} A_{P}=z K_{P} A_{P}=\gamma^{\prime} \bar{y}^{\prime} z^{\prime} K_{P} A_{P}=\gamma^{\prime} \bar{y} z K_{P} A_{P}
$$

so $\gamma^{-1} \gamma^{\prime} \in(\bar{y} z) K_{P} A_{P}(\bar{y} z)^{-1}$. Since $\Gamma_{L}$ is torsion-free, this implies $\gamma=\gamma^{\prime}$, hence the characteristic element $e=\gamma \bar{y}$ is unchanged. This proves (2). Since $\Gamma_{L}$ is discrete, the characteristic element is constant in a continuous family of fixed points, which proves (4).

Now consider changing $y$ within its double coset $\Gamma_{P} y \Gamma_{P}$ and consider changing the lift $\tilde{w} \in D_{P}$ of the fixed point. Let $\hat{y}=\gamma_{1} y \gamma_{2}$ with $\gamma_{1}, \gamma_{2} \in \Gamma_{P}$. Set $\bar{\gamma}_{1}=v_{P}\left(\gamma_{1}\right), \bar{\gamma}_{2}=v_{P}\left(\gamma_{2}\right)$ and $\overline{\hat{y}}=v_{P}(\hat{y})$. As in $\$ 8.1$, the element $\overline{\hat{y}}$ determines a Hecke correspondence $\left(\hat{c}_{1}, \hat{c}_{2}\right): \hat{C}_{P} \rightrightarrows X_{P}$ as follows: Set $\hat{\Gamma}_{P}=\Gamma_{P} \cap \hat{y}^{-1} \Gamma_{P} \hat{y}, \hat{\Gamma}_{L}=v_{P}\left(\hat{\Gamma}_{P}\right)$ and $\hat{C}_{P}=\hat{\Gamma}_{L} \backslash D_{P}=\hat{\Gamma}_{L} \backslash L_{P} / K_{P} A_{P}$. Then $\left(\hat{c}_{1}, \hat{c}_{2}\right)\left(\hat{\Gamma}_{L} x K_{P} A_{P}\right)=\left(\Gamma_{L} x K_{P} A_{P}, \Gamma_{L} \overline{\hat{y}} x K_{P} A_{P}\right)$ for any $x \in L_{P}$. As in equation (6.7.1), an isomorphism of correspondences $f: \hat{C}_{P} \rightarrow C_{P}$ is given by

$$
f\left(\hat{\Gamma}_{L} x K_{P} A_{P}\right)=\Gamma_{L}^{\prime} \bar{\gamma}_{2} x K_{P} A_{P}
$$

Choose any lift $\hat{z} K_{P} A_{P} \in D_{P}$ of the fixed point $\hat{w}=f^{-1}(w)$ (with $\hat{z} \in L_{P}$ ). We obtain a new characteristic element $\hat{e}=\hat{\gamma} \overline{\hat{y}}$ (for some $\hat{\gamma} \in \Gamma_{L}$ ) such that

$$
\hat{\gamma} \overline{\hat{y}} \hat{z} K_{P} A_{P}=\hat{z} K_{P} A_{P}
$$

We need to show that $\hat{e}=\hat{\gamma} \overline{\hat{y}}$ is $\Gamma_{L}$-conjugate to $e=\gamma \bar{y}$. Since $f(\hat{w})=w$ we have

$$
\Gamma_{L}^{\prime} \bar{\gamma}_{2} \hat{z} K_{P} A_{P}=\Gamma_{L}^{\prime} z K_{P} A_{P}
$$

so there exists a unique $h \in \Gamma_{L}^{\prime}$ such that $h \bar{\gamma}_{2} \hat{z} K_{P} A_{P}=z K_{P} A_{P}$ or

$$
\hat{z} K_{P} A_{P}=\bar{\gamma}_{2}^{-1} h^{-1} z K_{P} A_{P} .
$$

Substituting (8.5.2) into both sides of (8.5.1) and using (8.2.2) gives

$$
h \bar{\gamma}_{2} \hat{\gamma} \overline{\hat{y}} \bar{\gamma}_{2}^{-1} h^{-1} z K_{P} A_{P}=z K_{P} A_{P}=\gamma \bar{y} z K_{P} A_{P}
$$

or

$$
h \bar{\gamma}_{2} \hat{\gamma} \bar{\gamma}_{1} \bar{y} h^{-1} z K_{P} A_{P}=\gamma \bar{y} z K_{P} A_{P} .
$$


Since $h, h^{-1} \in v_{P}\left(y^{-1} \Gamma_{P} y\right)=\bar{y}^{-1} \Gamma_{L} \bar{y}$ there exists $h^{\prime} \in \Gamma_{L}$ such that $\bar{y} h^{-1}=h^{\prime} \bar{y}$, which gives

$$
\gamma^{-1} h \bar{\gamma}_{2} \hat{\gamma} \bar{\gamma}_{1} h^{\prime}\left(\bar{y} z K_{P} A_{P}\right)=\bar{y} z K_{P} A_{P}
$$

This implies that $\gamma^{-1} h \bar{\gamma}_{2} \hat{\gamma} \bar{\gamma}_{1} h^{\prime}=1$ since it is both in the group $(\bar{y} z) K_{P} A_{P}(\bar{y} z)^{-1}$ and in $\Gamma_{L}$. Therefore $1 . \bar{y}=\gamma^{-1} h \bar{\gamma}_{2} \hat{\gamma} \bar{\gamma}_{1} \bar{y} h^{-1}$ or

$$
\gamma \bar{y}=h \bar{\gamma}_{2} \hat{\gamma} \bar{\gamma}_{1} \bar{y}_{\gamma_{2}} \bar{\gamma}_{2}^{-1} h^{-1}=\left(h \bar{\gamma}_{2}\right) \hat{\gamma} \overline{\hat{y}}\left(h \bar{\gamma}_{2}\right)^{-1}
$$

Thus, the characteristic elements $\gamma \bar{y}$ and $\hat{\gamma} \overline{\hat{y}}$ are $\Gamma_{L}$-conjugate, which proves (3).

Now let us prove (5). It is easy to see that $L_{e}$ acts on $D_{P}^{e}$. To see that this action is transitive, let $v_{1}, v_{2} \in D_{P}^{e}$, say $v_{1}=z_{1} K_{P} A_{P}$ and $v_{2}=z_{2} K_{P} A_{P}$ with $z_{i} \in L_{P} \quad($ for $i=1,2)$. Then there exists $k_{1}, k_{2} \in K_{P} A_{P}$ so that $e z_{1}=z_{1} k_{1}$ and $e z_{2}=z_{2} k_{2}$, hence $k_{1}$ and $k_{2}$ are $L$ conjugate (by $z_{2} z_{1}^{-1}$ ). It follows from [Bo3], 24.7 that $k_{1}$ and $k_{2}$ are also $K_{P} A_{P}$ conjugate. Say, $k_{2}=m k_{1} m^{-1}$ for some $m \in K_{P} A_{P}$. Define $x=z_{2} m z_{1}^{-1}$. Then $v_{2}=x v_{1}$ and moreover, $x \in L_{e}$ since

$$
x e x^{-1}=z_{2} m z_{1}^{-1} e z_{1} m^{-1} z_{2}^{-1}=z_{2} m k_{1} m^{-1} z_{2}^{-1}=e .
$$

This completes the verification that $L_{e}$ acts transitively on $D_{P}^{e}$.

Using the chosen lift $\tilde{w}=z K_{P} A_{P} \in D_{P}^{e}$ as a basepoint, we obtain a diffeomorphism $L_{e} / K_{e}^{\prime} \cong D_{P}^{e}$ where $K_{e}^{\prime}=L_{e} \cap\left(z K_{P} A_{P} z^{-1}\right)$ is the stabilizer (in $\left.L_{e}\right)$ of $\tilde{w}$. This induces a surjection $\left(L_{e} \cap \Gamma_{L}^{\prime}\right) \backslash L_{e} / K_{e}^{\prime} \rightarrow F_{P}(e)$ which we will now show to be injective.

Suppose $x_{1}, x_{2} \in L_{e}$ and that $x_{1} \tilde{w}, x_{2} \tilde{w} \in D_{P}^{e}$ map to the same point in $C_{P}$, that is, $\Gamma_{L}^{\prime} x_{1} z K_{P} A_{P}=\Gamma_{L}^{\prime} x_{2} z K_{P} A_{P}$. Then there exists $\gamma \in \Gamma_{L}^{\prime}$ so that

$$
\gamma x_{1} z K_{P} A_{P}=x_{2} z K_{P} A_{P}
$$

We need to show that $\gamma \in L_{e}$. Acting by $e$ on the left hand side of (8.5.3) and using (8.2.2) gives the quantity

$$
e \gamma e^{-1} e x_{1} z K_{P} A_{P}=e \gamma e^{-1} x_{1} e z K_{P} A_{P}=e \gamma e^{-1} x_{1} z K_{P} A_{P}
$$

while acting by $e$ on the right hand side of (8.5.3) gives

$$
\operatorname{ex}_{2} z K_{P} A_{P}=x_{2} z K_{P} A_{P}=\gamma x_{1} z K_{P} A_{P}
$$

So $\gamma^{-1} e \gamma e^{-1} \in\left(x_{1} z\right) K_{P} A_{P}\left(x_{1} z\right)^{-1}$. But $\gamma^{-1} e \gamma e^{-1} \in \Gamma_{L}$ so this element is trivial, that is, $\gamma e=e \gamma$, hence $\gamma \in L_{e}$. Therefore $F_{P}(e)=\Gamma_{e}^{\prime} \backslash D_{P}^{e}$. The equality $c_{1}\left(F_{P}(e)\right)=c_{2}\left(F_{P}(e)\right)=\Gamma_{e} \backslash L_{e} / K_{e}^{\prime}$ is similar. Equation (8.4.2) will be proven in \$8.6.

Now let us verify part (6). Suppose $e^{\prime \prime}=\gamma_{2} \bar{y} \gamma_{1} \in \mathbf{L}_{\mathbf{P}}(\mathbb{Q})$ is elliptic (modulo $A_{P}$ ). Then $e^{\prime \prime}$ is $\Gamma_{L}$-conjugate to the element $e^{\prime}=\gamma_{1} \gamma_{2} \bar{y}$ which is also elliptic modulo $A_{P}$. There exists $z \in L_{P}$ so that $e^{\prime} \in z K_{P} A_{P} z^{-1}$ hence $e^{\prime} z K_{P} A_{P}=z K_{P} A_{P}$. In other words, $e^{\prime}$ is a characteristic element for the point $w^{\prime}=\Gamma_{L}^{\prime} z K_{P} A_{P} \in C_{P}$ (which is easily seen to be fixed under the Hecke correspondence). 
8.6. Proof of (8.4.2). (We refer to the notation of $\S 8.4$ and $\S 8.5$.) Unfortunately the arithmetic group $\Gamma_{L}[\bar{y}]=\Gamma_{L} \cap \bar{y}^{-1} \Gamma_{L} \bar{y}$ may be larger than $\Gamma_{L}^{\prime}=v_{P}\left(\Gamma_{P} \cap y^{-1} \Gamma_{P} y\right)$, so the correspondence (8.1.1) is not necessarily a Hecke correspondence, but rather it is a covering of the following Hecke correspondence:

$$
\begin{aligned}
\left(\tilde{c}_{1}, \tilde{c}_{2}\right): \tilde{C}_{P}=\Gamma_{L}[\bar{y}] \backslash D_{P} & \rightrightarrows X_{P}, \\
\Gamma_{L}[\bar{y}] x K_{P} A_{P} & \mapsto\left(\Gamma_{L} x K_{P} A_{P}, \Gamma_{L} \bar{y} x K_{P} A_{P}\right) .
\end{aligned}
$$

This covering $\phi: \Gamma_{L}^{\prime} \backslash D_{P} \rightarrow \Gamma_{L}[\bar{y}] \backslash D_{P}$ has degree $d=\left[\Gamma_{L}[\bar{y}]: \Gamma_{L}^{\prime}\right]$. A point $w \in C_{P}$ is fixed iff the point $\phi(w) \in \tilde{C}_{P}$ is fixed.

Let $\tilde{F}_{P}(e) \subset \tilde{C}_{P}$ be the set of fixed points within this (smaller) Hecke correspondence with characteristic element $e$. We claim that the restriction of $\tilde{c}_{i}$ to $\tilde{F}_{P}(e) \subset \tilde{C}_{P}$ is a diffeomorphism: $\quad \tilde{F}_{P}(e) \cong c_{i}\left(F_{P}(e)\right) \quad($ for $i=1,2)$. As in (8.4.1) it is clear that $\tilde{F}_{P}(e) \cong\left(\Gamma_{L}[\bar{y}] \cap L_{e}\right) \backslash L_{e} / K_{e}^{\prime}$ so it suffices to verify that the inclusion $\Gamma_{L}[\bar{y}] \cap L_{e} \subset \Gamma_{e}$ is an isomorphism. If $\gamma_{1} \in \Gamma_{e}$ then $\gamma_{1}=e^{-1} \gamma_{1} e$. But the left side of this equation is in $\Gamma_{L}$ and the right side is in $\bar{y}^{-1} \Gamma_{L} \bar{y}$, hence $\gamma_{1} \in \Gamma_{L}[\bar{y}]$, which proves the claim.

In summary, we have a diagram

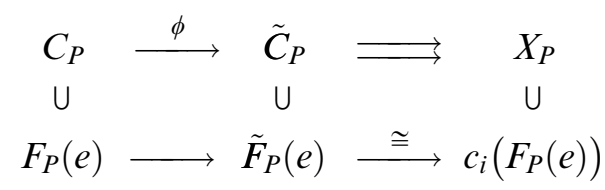

and in particular the degree $d$ of the covering $\phi$ coincides with the degree $d_{e}$ of the mapping $F_{P}(e) \rightarrow c_{i}\left(F_{P}(e)\right)$ which gives (8.4.2).

8.7. Remark. The codimension of $F_{p}(e)$ in $D_{P}$ is odd if and only if the action of $e$ reverses orientations in the normal bundle of $F_{P}(e)$ in $D_{P}$. This is because $e$ preserves an appropriately chosen normal slice through any point in $F_{P}(e)$, the boundary of which is a sphere on which $e$ then acts as a diffeomorphism without fixed points, so its Lefschetz number is 0 . In the odd codimension case this sphere is even dimensional, so by the Lefschetz fixed point theorem, its action on the top degree cohomology is given by multiplication by -1 , that is, it reverses the orientation. In the even codimension case, it preserves orientation.

\section{Hyperbolic properties of Hecke correspondences}

9.1. Expanding and contracting roots. As in $\S 2$, G denotes a connected linear reductive algebraic group defined over $\mathbb{Q}, D$ denotes the associated symmetric space, $K^{\prime}=A_{G} K\left(x_{0}\right)$ is the stabilizer in $G$ of a fixed basepoint $x_{0} \in D, \Gamma \subset \mathbf{G}(\mathbb{Q})$ is an arithmetic group and $X=\Gamma \backslash D$. Throughout this section we fix a Hecke correspondence $\left(c_{1}, c_{2}\right): \bar{C} \rightrightarrows \bar{X}$ defined by some element $g \in \mathbf{G}(\mathbb{Q})$. So $C=\Gamma^{\prime} \backslash D$ with $\Gamma^{\prime}=\Gamma \cap g^{-1} \Gamma g$.

Let $\mathbf{P} \subset \mathbf{G}$ be a rational parabolic subgroup and suppose that $c_{1}\left(C_{P}\right)=c_{2}\left(C_{P}\right)=X_{P}$. By Proposition 7.3, near $C_{P}$ the correspondence is modeled on a parabolic Hecke corre- 
spondence $\Gamma_{P}^{\prime} \backslash D[P] \rightrightarrows \Gamma_{P} \backslash D[P]$ (7.1.1) which is determined by some $y \in \mathbf{P}(\mathbb{Q})$ (where $\left.\Gamma_{P}^{\prime}=\Gamma_{P} \cap y^{-1} \Gamma_{P} y\right)$. Suppose $y=u_{y} a_{y} m_{y}$ is the Langlands decomposition (2.2.2) of $y \in \mathbf{P}(\mathbb{Q})$. If $y$ is allowed to vary within the double coset $\Gamma_{P} y \Gamma_{P}$ then the element $a_{y} \in A_{P}$ will remain fixed, so we may write $a_{P}=a_{y}$. We refer to $a_{P}$ as the torus factor associated to the Hecke correspondence near $C_{P}$. The torus factor may be used to define a partition of the simple roots $\Delta_{P}$ into three subsets

$$
\begin{aligned}
& \Delta_{P}^{+}=\left\{\alpha \in \Delta_{P} \mid \alpha\left(a_{P}\right)<1\right\}, \\
& \Delta_{P}^{-}=\left\{\alpha \in \Delta_{P} \mid \alpha\left(a_{P}\right)>1\right\}, \\
& \Delta_{P}^{0}=\left\{\alpha \in \Delta_{P} \mid \alpha\left(a_{P}\right)=1\right\},
\end{aligned}
$$

consisting of those simple roots which are expanding, contracting, or neutral, respectively, near the stratum $C_{P}$. (See also $§ 11.7$.) The terminology is motivated by the following fact, whose proof follows immediately from (3.2.2) and the definition (7.1.2) of the correspondence. For all $\alpha \in \Delta_{P}$ and for all $z \in \Gamma_{P}^{\prime} \backslash D[P]$ the root function $f_{\alpha}^{P}$ satisfies:

$$
f_{\alpha}^{P}\left(c_{2}(z)\right)=\alpha\left(a_{P}\right) f_{\alpha}^{P}\left(c_{1}(z)\right) .
$$

Now suppose $\mathbf{P} \subset \mathbf{Q}$ are rational parabolic subgroups of $\mathbf{G}$, with $\Delta_{P}=i\left(\Delta_{Q}\right) \amalg J$ as in (2.4.1). Suppose that $c_{1}\left(C_{P}\right)=c_{2}\left(C_{P}\right)$, giving rise to a torus factor $a_{P} \in A_{P}$ and a decomposition of $\Delta_{P}$ into expanding, contracting and neutral roots as above. Then $c_{1}\left(C_{Q}\right)=c_{2}\left(C_{Q}\right)$ (by $\left.\S 7.1\right)$ so we obtain a torus factor $a_{Q} \in A_{Q}$ and a decomposition of $\Delta_{Q}$ into expanding, contracting and neutral roots also.

9.2. Proposition. Suppose that $J \subset \Delta_{P}^{0}$. Then:

(1) The torus factors $a_{P}=a_{Q}$ are equal; in particular $a_{P}$ lies in the sub-torus $A_{Q} \subset A_{P}$.

(2) The expanding, contracting, and neutral simple roots for $\mathbf{P}$ and for $\mathbf{Q}$ are related as follows:

$$
\Delta_{P}^{+}=i\left(\Delta_{Q}^{+}\right), \quad \Delta_{P}^{-}=i\left(\Delta_{Q}^{-}\right), \quad \Delta_{P}^{0}=i\left(\Delta_{Q}^{0}\right) \amalg J .
$$

(3) For all $z \in \Gamma_{P}^{\prime} \backslash D[P]$ and for all $\beta \in \Delta_{Q}$ we have,

$$
\frac{f_{i(\beta)}^{P}\left(c_{2}(z)\right)}{f_{i(\beta)}^{P}\left(c_{1}(z)\right)}=i(\beta)\left(a_{P}\right)=\beta\left(a_{P}\right)=\frac{f_{\beta}^{Q}\left(c_{2}(z)\right)}{f_{\beta}^{Q}\left(c_{1}(z)\right)}
$$

provided the denominators do not vanish.

In this case we say that $\mathbf{Q}$ is a neutral parabolic subgroup containing $\mathbf{P}$ and we write $\mathbf{P} \prec \mathbf{Q}$. Intuitively, the Hecke correspondence is neutral in those directions normal to $C_{P}$ which point into $C_{Q}$; cf. $\$ 3.6$.

9.3. Proof. Locally near $C_{Q}$ the Hecke correspondence is isomorphic to a parabolic Hecke correspondence given by some $y^{\prime}=u_{y^{\prime}} a_{y^{\prime}} m_{y^{\prime}} \in Q$ with torus factor $a_{Q}=a_{y^{\prime}} \in A_{Q}$. In a neighborhood of $C_{P}$ the correspondence is isomorphic to the parabolic Hecke corre- 
spondence given by some $y=u_{y} a_{y} m_{y} \in P$ (with $a_{P}=a_{y}$ ). Moreover $y$ may be chosen to lie in the double coset $\Gamma_{Q} y^{\prime} \Gamma_{Q}$ since the correspondence $\bar{C}_{P} \rightrightarrows \bar{X}_{P}$ is the restriction to $\bar{C}_{P}$ of the correspondence $\bar{C}_{Q} \rightrightarrows \bar{X}_{Q}$; cf. Proposition 7.3. By assumption, $\alpha\left(a_{P}\right)=1$ for all $\alpha \in J$ which implies that $a_{P} \in A_{Q}$. It follows that $a_{P}=a_{Q}$ because the homomorphism $Q \rightarrow A_{Q}$ (which associates to any $z \in Q$ its torus factor $a_{z}$ ) kills $\Gamma_{Q}$. Therefore, for any $\beta \in \Delta_{Q}$ we have: $\beta\left(a_{Q}\right)=i(\beta)\left(a_{P}\right)$. This proves (1) and (2). The first equality in part (3) is just (9.1.1). The last equality in part (3) follows from part (1) and from (9.1.1) (with $f_{\alpha}^{P}$ replaced by $\left.f_{\beta}^{Q}\right)$.

9.4. Maximal neutrality. Suppose $\mathbf{P} \subset \mathbf{Q} \subset \mathbf{R}$ are rational parabolic subgroups and that $c_{1}\left(C_{P}\right)=c_{2}\left(C_{P}\right)$. Write $\Delta_{P}=i\left(\Delta_{Q}\right) \amalg I$ and $\Delta_{Q}=j\left(\Delta_{R}\right) \amalg J$ for the disjoint union of (2.4.1). Suppose moreover that $\mathbf{P} \prec \mathbf{Q}$ and $\mathbf{Q} \prec \mathbf{R}$, that is, that $I \subset \Delta_{P}^{0}$ and $J \subset \Delta_{Q}^{0}$. Then it follows from Proposition 9.2 that $\mathbf{P} \prec \mathbf{R}$. Hence there is a greatest neutral parabolic subgroup $\mathbf{P}^{\dagger}$ containing $\mathbf{P}$; in fact it is $\mathbf{P}^{\dagger}=\mathbf{P}\left(\Delta_{P}^{0}\right)$ in the notation of $\S 2.4$ and $\S 2.3$. It is easy to see that

$$
\Delta_{P^{\dagger}}^{0}=\phi \quad \text { and } \quad \Delta_{P}^{ \pm}=i^{\prime}\left(\Delta_{P^{\dagger}}^{ \pm}\right)
$$

(where $i^{\prime}: \Delta_{P^{\dagger}} \hookrightarrow \Delta_{P}$ is the natural inclusion). Moreover,

$$
\mathbf{P} \prec \mathbf{Q} \Rightarrow \mathbf{P}^{\dagger}=\mathbf{Q}^{\dagger} .
$$

\section{Structure of the fixed point set}

10.1. As in $\S 2$, $\mathbf{G}$ denotes a connected reductive linear algebraic group defined over $\mathbb{Q}, D=G / K^{\prime}$ is its associated symmetric space with basepoint $x_{0} \in D$ and stabilizer $K^{\prime}=A_{G} K\left(x_{0}\right), \Gamma \subset \mathbf{G}(\mathbb{Q})$ denotes an arithmetic subgroup, and $X=\Gamma \backslash D$. Throughout this section we fix a Hecke correspondence $\left(c_{1}, c_{2}\right): \bar{C} \rightrightarrows \bar{X}$ defined by some element $g \in \mathbf{G}(\mathbb{Q})$. So $C=\Gamma^{\prime} \backslash D$ with $\Gamma^{\prime}=\Gamma \cap g^{-1} \Gamma g$. We also fix a $\Gamma$-equivariant tiling of $D$ which is narrow with respect to the Hecke correspondence (cf. $\S 6.11, \S 4.5$ ), and denote by $\left\{C^{P}\right\}$ and $\left\{X^{P}\right\}$ the resulting tilings of $\bar{C}$ and $\bar{X}$ respectively. Let $F \subset \bar{C}$ be a connected component of the set of fixed points. The following lemma says that if $F$ spans two strata $C_{P} \subset \bar{C}_{Q}$ then the Hecke correspondence is neutral in those directions which point from $C_{P}$ into $C_{Q}$; cf. §3.6.

10.2. Lemma. Let $\mathbf{P} \subset \mathbf{Q} \cong \mathbf{G}$ be rational parabolic subgroups and write $\Delta_{P}=i\left(\Delta_{Q}\right) \amalg J$ as in (2.4.1). Suppose $F \cap C_{Q} \cap T\left(\bar{C}_{P}\right) \neq \emptyset$ (that is, $C_{Q}$ contains fixed points which lie in the $\Gamma$-parabolic neighborhood $T\left(\bar{C}_{P}\right)$ of $\left.\bar{C}_{P}\right)$. Then:

(1) $J \subset \Delta_{P}^{0}$ (hence the conclusions of Proposition 9.2 hold).

(2) $F \cap T\left(\bar{C}_{P}\right)$ is invariant under the geodesic action of $A_{P}^{\prime}(\geqq 1)$.

(3) $\pi_{P}\left(F \cap T\left(\bar{C}_{P}\right)\right) \subset F$, that is, each fixed point in this $\Gamma^{\prime}$-parabolic neighborhood projects to a fixed point in $C_{P}$.

10.3. Proof. Part (1) follows from (3.5.2) and (9.1.1) by taking $z \in F \cap C_{Q} \cap T\left(\bar{C}_{P}\right)$ to be a fixed point. Part (2) follows from (7.1.3). Part (3) follows by continuity. 
10.4. Proposition. Let $F \subset \bar{C}$ be a connected component of the fixed point set. Let $\mathbf{Q}$ be a rational parabolic subgroup and suppose $F \cap C_{Q} \neq \emptyset$. Let $a_{Q} \in A_{Q}$ be the torus factor for this stratum. Let $\mathbf{Q}^{\dagger}=\mathbf{Q}\left(\Delta_{Q}^{0}\right)$ be the maximal neutral parabolic subgroup containing $\mathbf{Q}$. Then:

(1) The whole connected component F of the fixed point set is contained in the closure

$$
F \subset \bar{C}_{Q^{\dagger}}
$$

of the single stratum $C_{Q^{\dagger}}$.

(2) The Hecke correspondence $\bar{C} \rightrightarrows \bar{X}$ restricts to a correspondence $\bar{C}_{Q^{\dagger}} \rightrightarrows \bar{X}_{Q^{\dagger}}$ on this stratum-closure. Within this restricted correspondence, near each point $c \in F$, every simple root is neutral: If $\mathbf{P} \subset \mathbf{Q}^{\dagger}$, if $F \cap C_{P} \neq \emptyset$, if $i^{\prime}: \Delta_{Q^{\dagger}} \hookrightarrow \Delta_{P}$ is the inclusion, and if $\Delta_{P}=i^{\prime}\left(\Delta_{Q^{\dagger}}\right) \amalg J$ as in (2.4.1) then $J=\Delta_{P}^{0}$.

(3) There exists a neighborhood $U(F) \subset \bar{C}$ of the fixed point set such that for all $\alpha \in \Delta_{Q}$ and for all $x \in U(F)$, if $x \in T\left(\bar{C}_{Q}\right)$ and if $c_{2}(x) \in T\left(\bar{X}_{Q}\right)$ then

$$
r_{\alpha}^{Q}\left(c_{2}(x)\right)=\alpha\left(a_{Q}\right)^{-1} r_{\alpha}^{Q}\left(c_{1}(x)\right)
$$

10.5. Remarks. Part (1) does not imply that $F \cap C_{Q^{\dagger}} \neq \emptyset$. In fact, the fixed point component $F$ may be "reducible": it does not necessarily coincide with the closure of its intersection $F \cap C_{P}$ with any single stratum $C_{P}$. (See $\$ 16.1$.)

10.6. Proof. Suppose that $F$ has a nontrivial intersection with some other stratum, say $F \cap C_{R} \neq \emptyset$. Suppose for the moment that $\mathbf{R} \supset \mathbf{Q}$ and that $F \cap C_{Q}$ contains limit points from $F \cap C_{R}$, that is,

$$
\left(F \cap C_{Q}\right) \cap \overline{F \cap C_{R}} \neq \emptyset .
$$

Then Lemma 10.2 part (1) implies that $\mathbf{R}$ is a neutral parabolic subgroup containing $\mathbf{Q}$ so (9.4.2) implies that $\mathbf{R}^{\dagger}=\mathbf{Q}^{\dagger}$, hence $F \cap C_{R} \subset F \cap \bar{C}_{R^{\dagger}}=F \cap \bar{C}_{Q^{\dagger}}$. Now we drop the assumption (10.6.1). Since $F$ is connected, the stratum $C_{R}$ is related to the stratum $C_{Q}$ through a chain of strata $C_{R_{i}}$ (say, $1 \leqq i \leqq m$ ), each having nontrivial intersection with $F$, with each step in the chain related to the next by

$$
\left(F \cap C_{R_{i}}\right) \cap \overline{F \cap C_{R_{i+1}}} \neq \emptyset \quad \text { or } \quad\left(F \cap C_{R_{i+1}}\right) \cap \overline{F \cap C_{R_{i}}} \neq \emptyset .
$$

Repeated application of (9.4.2) implies that

$$
\mathbf{R}^{\dagger}=\mathbf{R}_{1}^{\dagger}=\cdots=\mathbf{R}_{m}^{\dagger}=\mathbf{Q}^{\dagger}
$$

So once again, $F \cap C_{R} \subset F \cap \bar{C}_{Q^{\dagger}}$. This verifies part (1).

Consider part (2). Since the stratum $C_{Q}$ is preserved by the Hecke correspondence, the same holds for each larger stratum, especially $C_{Q^{\dagger}}$. Suppose $F \cap C_{P} \neq \emptyset$. By (10.6.2), $\mathbf{P}^{\dagger}=\mathbf{Q}^{\dagger}$ so by $(9.4 .1), \Delta_{P}=i^{\prime}\left(\Delta_{Q^{\dagger}}\right) \amalg \Delta_{P}^{0}$. 
Next we verify part (3). Suppose $\mathbf{P} \subset \mathbf{Q}$ and suppose $F \cap C_{P} \neq \emptyset$. By Proposition 9.2 part (3), for all $\alpha \in \Delta_{Q}$ and for all $w \in T\left(\bar{C}_{Q}\right) \cap c_{2}^{-1}\left(T\left(\bar{X}_{Q}\right)\right)$, the root function $f_{i(\alpha)}^{P}$ satisfies

$$
f_{i(\alpha)}^{P}\left(c_{2}(w)\right)=\alpha\left(a_{Q}\right) f_{i(\alpha)}^{P}\left(c_{1}(w)\right)
$$

where, as in (2.4.1), we have written $\Delta_{P}=i\left(\Delta_{Q}\right) \amalg J$. However this does not yet prove (10.4.1). The problem is that the partial distance function $r_{\alpha}^{Q}(w)$ is patched together (4.2.2) from these root functions $f_{i(\alpha)}^{P}$ in a way that depends on which tile contains the point $c_{i}(w)$. So we need to show that the Hecke correspondence preserves the tile boundaries in some neighborhood $U(F)$ of the fixed point set. This in turn will follow from the neutrality properties of Lemma 10.2 and Proposition 9.2.

10.7. Lemma. Suppose $F \subset \bar{C}$ is a connected component of the fixed point set of the Hecke correspondence $\bar{C} \rightrightarrows \bar{X}$. Then there exists a neighborhood $U(F) \subset \bar{C}$ of $F$ such that for all $w \in U(F)$ and for any rational parabolic subgroup $\mathbf{P} \subseteq \mathbf{G}$,

$$
c_{1}(w) \in X^{P} \Leftrightarrow c_{2}(w) \in X^{P} .
$$

10.8. Proof. Assume not. Then there is a sequence of points $x_{i} \in \bar{C}$ converging to $F$ so that for each $i, c_{1}\left(x_{i}\right)$ and $c_{2}\left(x_{i}\right)$ are in different tiles. By taking subsequences if necessary we may assume the sequence $x_{i}$ converges to some point $x_{0} \in F$, that $x_{i}$ are all contained in a single tile $C^{P}$ (so $c_{1}\left(x_{i}\right) \in X^{P}$ ) and that $c_{2}\left(x_{i}\right)$ all lie in a single tile $X^{Q}$. Since $c_{1}\left(x_{0}\right) \in \bar{C}^{P} \cap \bar{C}^{Q}$ is a fixed point, the Hecke correspondence must preserve the strata $C_{P}$ and $C_{Q}$ (meaning that $c_{1}\left(C_{P}\right)=c_{2}\left(C_{P}\right)=X_{P}$ and $\left.c_{1}\left(C_{Q}\right)=c_{2}\left(C_{Q}\right)=X_{Q}\right)$ and we may assume that either $\mathbf{P} \cong \mathbf{Q}$ or $\mathbf{Q} \cong \mathbf{P}$. Since the tiling is narrow this implies that $F \cap C_{P} \neq \emptyset$, that $F \cap C_{Q} \neq \emptyset$, and that either $F \cap C_{P}$ contains limit points from $F \cap C_{Q}$ (if $\mathbf{P} \subseteq \mathbf{Q}$ ) or else $F \cap C_{Q}$ contains limit points from $F \cap C_{P}$ (if $\mathbf{Q} \cong \mathbf{P}$ ).

Let us first consider the case that $\mathbf{P} \cong \mathbf{Q}=\mathbf{G}$, meaning that $X^{Q}=X^{0}$. Let $a_{P} \in A_{P}$ denote the torus factor for the Hecke correspondence near $C_{P}$ as in $\$ 9.1$. Then it follows from Lemma 10.2 that $\mathbf{Q}=\mathbf{G}$ is a neutral parabolic subgroup containing $\mathbf{P}$, that is, $\alpha\left(a_{P}\right)=1$ for all $\alpha \in \Delta_{P}$. Within any $\Gamma$-parabolic neighborhood $W$ of $\bar{X}_{P}$, the tile $X^{P}$ is given by (4.1.3):

$$
X^{P}=\left\{x \in W \mid \pi_{P}(x) \in X_{P}^{0} \text { and } f_{\alpha}^{P}(x)>\alpha\left(b_{P}\right) \text { for all } \alpha \in \Delta_{P}\right\} .
$$

Since $c_{2}\left(x_{i}\right) \in X^{0}$ it follows that for at least one $\alpha \in \Delta_{P}$ we have:

$$
\alpha\left(b_{P}\right) \geqq f_{\alpha}^{P}\left(c_{2}\left(x_{i}\right)\right)=\alpha\left(a_{P}\right) f_{\alpha}^{P}\left(c_{1}\left(x_{i}\right)\right)=f_{\alpha}^{P}\left(c_{1}\left(x_{i}\right)\right)>\alpha\left(b_{P}\right)
$$

(using equation (9.1.1)), which is a contradiction.

Next consider the case $\mathbf{P} \subset \mathbf{Q} \neq \mathbf{G}$. For sufficiently large $i$ the points $x_{i}$ will lie in some $\Gamma^{\prime}$-parabolic neighborhood of $\bar{C}_{Q}$, and the same argument applied to the sequence $z_{i}=\pi_{Q}\left(x_{i}\right) \rightarrow z_{0}=\pi_{Q}\left(x_{0}\right) \in F \cap C_{Q}$ also leads to a contradiction.

The case $\mathbf{Q} \cong \mathbf{P}$ may be handled by reversing the roles of $\mathbf{P}$ and $\mathbf{Q}$ in these arguments. This completes the proof of Lemma 10.7 and also the proof of Proposition 10.4. 


\section{Modified Hecke correspondence}

11.1. As in $\S 2, \mathbf{G}$ denotes a connected linear reductive algebraic group defined over $\mathbb{Q}, D$ denotes the associated symmetric space, $K^{\prime}=A_{G} K\left(x_{0}\right)$ is the stabilizer in $G$ of a chosen basepoint $x_{0} \in D, \Gamma \subset \mathbf{G}(\mathbb{Q})$ is an arithmetic group and $X=\Gamma \backslash D$. Throughout this section we fix a Hecke correspondence $\left(c_{1}, c_{2}\right): \bar{C} \rightrightarrows \bar{X}$ defined by some element $g \in \mathbf{G}(\mathbb{Q})$, with $C=\Gamma^{\prime} \backslash D$ and $\Gamma^{\prime}=\Gamma \cap g^{-1} \Gamma g$. Let $F=\left\{w \in \bar{C} \mid c_{1}(w)=c_{2}(w)\right\}$ denote the fixed point set. Fix a sufficiently large regular $\Gamma$-equivariant parameter $b \in \mathscr{B}$ which is so large that the resulting tilings $\left\{D^{P}\right\}$ of $\bar{D},\left\{X^{P}\right\}$ of $\bar{X}$ and $\left\{C^{P}\right\}$ of $\bar{C}$ are narrow (\$6.11) with respect to the Hecke correspondence. Fix $\mathbf{t} \in A_{P_{0}}(>1)$ dominant and regular, with resulting shrink homeomorphism $\operatorname{Sh}(\mathbf{t}): \bar{X} \rightarrow \bar{X}$ as in $\S 5$. Define the (shrink-) modified correspondence

$$
\left(c_{1}^{\prime}, c_{2}^{\prime}\right): \bar{C} \rightrightarrows \bar{X}
$$

by $c_{1}^{\prime}=c_{1}$ and $c_{2}^{\prime}=S h(\mathbf{t}) \circ c_{2}$. Let $\hat{F}=\left\{w \in \bar{C} \mid c_{1}^{\prime}(w)=c_{2}^{\prime}(w)\right\}$ denote the fixed point set of the modified correspondence.

11.2. Proposition. If $\mathbf{t} \in A_{P_{0}}(>1)$ is chosen regular and sufficiently close to $\mathbf{1}$, then

$$
\hat{F} \cap C_{Q}=F \cap C_{Q}^{0}
$$

for each stratum $C_{Q} \subset \bar{C}$, and

$$
c_{1}(\hat{F}) \cap X_{Q}=c_{1}(F) \cap X_{Q}^{0}
$$

for each stratum $X_{Q} \subset \bar{X}$, where $C_{Q}^{0}\left(\right.$ resp. $\left.X_{Q}^{0}\right)$ denotes the central tile in $C_{Q}$ (resp. $\left.X_{Q}\right)$.

11.3. Proof. The correspondence $\bar{C}$ has finitely many boundary strata $C_{P}$ with the property that $c_{1}\left(C_{P}\right)=c_{2}\left(C_{P}\right)$. For each such stratum $C_{P}$, according to Proposition 7.3 , the Hecke correspondence is locally isomorphic near $C_{P}$ to a parabolic Hecke correspondence $\Gamma_{P}^{\prime} \backslash D[P] \rightrightarrows \Gamma_{P} \backslash D[P]$ which is given by some $y \in P(\mathbb{Q})$ and to which we may uniquely associate a torus factor $a_{y}=a_{P} \in A_{P}$ as in $\S 7.1$. Conjugating all these torus factors back to $\mathbf{S}_{\mathbf{P}_{\mathbf{0}}}$ gives a collection $\left\{a_{1}, a_{2}, \ldots, a_{N}\right\} \subset A_{P_{0}}$ of finitely many standard torus factors (some of which may coincide and some of which may equal 1) associated to the Hecke correspondence $g$. If $\mathbf{t} \in A_{P_{0}}(>1)$ is chosen to be regular and sufficiently close to $\mathbf{1}$ then we can guarantee that the following condition holds: For all $\alpha \in \Delta$ and for all $i=1,2, \ldots, N$, if $\alpha\left(a_{i}\right)<1$ then $\alpha\left(a_{i} \mathbf{t}\right)<1$ while if $\alpha\left(a_{i}\right) \geqq 1$ then $\alpha\left(a_{i} \mathbf{t}\right)>1$. Therefore, for any $\rho$ with $0<\rho \leqq 1$, for all $\alpha \in \Delta$ and for all $i=1,2, \ldots, N$, the following holds:

$$
\left\{\begin{array}{l}
\alpha\left(a_{i}\right)<1 \Rightarrow \alpha\left(a_{i}\right) \alpha(\mathbf{t})^{\rho}<1, \\
\alpha\left(a_{i}\right) \geqq 1 \Rightarrow \alpha\left(a_{i}\right) \alpha(\mathbf{t})^{\rho}>1 .
\end{array}\right.
$$

Having made these choices, let us now prove Proposition 11.2. Certainly $F \cap C_{Q}^{0}=\hat{F} \cap C_{Q}^{0}$ because the shrink acts as the identity on $C_{Q}^{0}$. So we only need to show that $\hat{F} \cap C_{Q} \subset C_{Q}^{0}$, that is, we must show that the fixed points of the modified Hecke correspondence which appear in the stratum $C_{Q}$ are all contained in the central tile of that stratum. Suppose otherwise and let $w \in C_{Q}$ be a fixed point of the modified correspondence which lies in 
some tile $C^{P}$ for $\mathbf{P} \subset \mathbf{Q}$, and $\mathbf{P} \neq \mathbf{Q}$. Since the shrink preserves tiles, it follows that $c_{1}\left(C^{P}\right) \cap c_{2}\left(C^{P}\right) \neq \emptyset$. The tiling is narrow so this implies that $c_{1}\left(C_{P}\right)=c_{2}\left(C_{P}\right)$. Set $\Delta_{P}=i\left(\Delta_{Q}\right) \amalg J$ as in $(2.4 .1)$; then $J \neq \emptyset$. By Proposition 7.3, locally near $C_{P}$ we may replace the Hecke correspondence by a parabolic correspondence: in other words, we may assume that $g \in \mathbf{P}$. Let $a_{P} \in A_{P}$ be the torus factor for the correspondence near $C_{P}$, that is, if $g=u_{g} a_{g} m_{g} \in \mathscr{U}_{P} A_{P} M_{P}$ is the Langlands decomposition then $a_{P}=a_{g}$. The point $c_{1}(w)=c_{1}^{\prime}(w)=c_{2}^{\prime}(w)$ lies in $X_{Q} \cap X^{P} \subset \bar{X}$. Since the shrink preserves tiles, $c_{2}(w) \in X_{Q} \cap X^{P}$ also.

For any $\alpha \in \Delta_{P}$,

$$
\begin{array}{rlrl}
f_{\alpha}^{P}\left(c_{2}^{\prime}(w)\right) & =f_{\alpha}^{P}\left(c_{2}(w)\right) \alpha_{0}(\mathbf{t})^{\rho\left(r_{\alpha}^{P}\left(c_{2}(w)\right)\right)} & & \text { by }(5.5 .2) \\
& =\alpha\left(a_{P}\right) \alpha_{0}(\mathbf{t})^{\rho\left(r_{\alpha}^{P}\left(c_{2}(w)\right)\right)} f_{\alpha}^{P}\left(c_{1}(w)\right) & \text { by }(9.1 .1)
\end{array}
$$

where $\alpha_{0} \in \Delta$ is the unique simple root which, after conjugation and restriction to $\mathbf{S}_{\mathbf{P}}$, agrees with $\alpha$.

This gives a contradiction: First note that $\rho\left(r_{\alpha}^{P}\left(c_{2}(w)\right)\right) \neq 0$, for otherwise we would have $r_{\alpha}^{P}\left(c_{2}(w)\right)=1$ or $c_{2}(w) \notin X^{P}$. As the shrink preserves tiles, this would imply that $c_{2}^{\prime}(w) \notin X^{P}$ which is absurd. So by $(11.3 .1)$ the factor $\alpha\left(a_{P}\right) \alpha_{0}(\mathbf{t})^{\rho\left(r_{\alpha}^{P}\left(c_{2}(w)\right)\right)} \neq 1$. If we choose $\alpha \in J$ then the assumption $c_{1}(w) \in X_{Q}$ implies that $f_{\alpha}^{P}\left(c_{1}(w)\right) \neq 0$. Therefore the point $w$ cannot be fixed by the modified correspondence, which proves (11.2.1).

There are finitely many strata $C_{R}$ such that $c_{1}\left(C_{R}\right)=X_{Q}$. To prove (11.2.2) it suffices to show, for each of these strata, that

$$
c_{1}\left(\hat{F} \cap C_{R}\right) \cap X_{Q}=c_{1}\left(F \cap C_{R}\right) \cap X_{Q}^{0}
$$

Write $F \cap C_{R}=\left(F \cap C_{R}^{0}\right) \cup \tilde{F}$ as a disjoint union. Then $\tilde{F}$ is contained in a union of tiles $C^{R^{\prime}}$ with $\mathbf{R}^{\prime} \subset \mathbf{R}$ a proper inclusion. Since $c_{1}$ takes tiles to tiles, it follows that $c_{1}(\tilde{F}) \cap X_{Q}^{0}=\emptyset$ hence

$$
\begin{aligned}
c_{1}\left(F \cap C_{R}\right) \cap X_{Q}^{0}=c_{1}\left(F \cap C_{R}^{0}\right) \cap X_{Q}^{0} & =c_{1}\left(F \cap C_{R}^{0}\right) \\
& =c_{1}\left(\hat{F} \cap C_{R}\right)=c_{1}\left(\hat{F} \cap C_{R}\right) \cap X_{Q}
\end{aligned}
$$

by $(11.2 .1)$.

11.4. Tangential distance. Choose a regular $\Gamma$-invariant parameter so that the associated tiling is narrow (\$6.11) with respect to the Hecke correspondence. Choose $\mathbf{t} \in A_{P_{0}}(>1)$ to be regular and sufficiently close to 1 as in Proposition 11.2. Suppose $C_{Q}$ is a stratum of $\bar{C}$ for which $F \cap C_{Q} \neq \emptyset$. Then the Hecke correspondence restricts to a correspondence $C_{Q} \rightrightarrows X_{Q}$. Fix $e \in L_{Q}$ and let $F_{Q}(e) \subset C_{Q}$ denote the set of fixed points in $C_{Q}$ for which $e$ is a characteristic element as in $\S 8.2$. The corresponding set of fixed points for the modified Hecke correspondence is the "truncation"

$$
F_{Q}^{0}(e)=F_{Q}(e) \cap C_{Q}^{0} .
$$


Let $E=c_{1}\left(F_{Q}^{0}(e)\right)=c_{1}\left(F_{Q}(e)\right) \cap X_{Q}^{0}$ denote its image in $X_{Q}$. In this section we construct a good function which measures the distance from $E$.

Let $R_{X_{Q}}: X_{Q} \rightarrow X_{Q}^{0}$ and $R_{C_{Q}}: C_{Q} \rightarrow C_{Q}^{0}$ be the retraction(s) and let $W_{Q}: \overline{X_{Q}} \rightarrow[0,1]$ be the exhaustion function of $\$ 4.6$. A choice of $G$-invariant Riemannian metric on $D$ induces Riemannian metrics on $C, X, C_{Q}$, and $X_{Q}$. Define the tangential distance $d_{E}: X_{Q} \rightarrow[0, \infty]$ by

$$
d_{E}(x)=W_{Q}(x)+\operatorname{dist}_{X_{Q}}\left(R_{X_{Q}}(x), E\right)
$$

where dist $_{X_{Q}}$ denotes the distance in $X_{Q}$ with respect to the Riemannian metric. Then $d_{E}^{-1}(0)=E$. Although the restriction of the Hecke correspondence to $\bar{C}_{Q}$ is locally an isometry, composing with $S h(\mathbf{t})$ has the following effect: points near the boundary of $X_{Q}$ are moved even closer to the boundary of $X_{Q}$ and hence they are moved away from $E$. This is the intuition behind the following lemma.

11.5. Lemma. There exists a neighborhood $V \subset C_{Q}$ of $F_{Q}^{0}(e)=F_{Q}(e) \cap C_{Q}^{0}$ such that

$$
d_{E}\left(c_{2}^{\prime}(w)\right) \geqq d_{E}\left(c_{1}^{\prime}(w)\right)
$$

for all $w \in V$.

11.6. Proof. The stratum closure $\bar{C}_{Q}$ is tiled by the collection of intersections $C_{Q}^{P}=\bar{C}_{Q} \cap C^{P}$ with $\mathbf{P} \subseteq \mathbf{Q}$. Let $\bar{C}_{Q}^{P}$ denote the closure of such a tile. Let $U_{1} \subset \bar{C}_{Q}$ be a neighborhood of the closure $\bar{F}_{Q}(e)$ so that for any rational parabolic subgroup $\mathbf{P} \subset \mathbf{Q}$,

$$
\bar{F}_{Q}(e) \cap \bar{C}_{Q}^{P}=\emptyset \Leftrightarrow U_{1} \cap \bar{C}_{Q}^{P}=\emptyset .
$$

By Lemma 10.7 we may also assume that the Hecke correspondence preserves tile boundaries in $U_{1}$. The mapping $c_{1}$ preserves tiles, and the points in $F_{Q}(e)$ are fixed, hence

$$
E=c_{1}\left(F_{Q}^{0}(e)\right)=c_{2}\left(F_{Q}^{0}(e)\right)
$$

By Proposition $8.4(6)$, and for $i=1,2$, the mapping $c_{i}$ is one-to-one on $F_{Q}(e)$. Moreover, it is locally an isometry. It follows that,

$$
\operatorname{dist}_{X_{Q}}\left(c_{1}(w), E\right)=\operatorname{dist}_{C_{Q}}\left(w, F_{Q}^{0}(e)\right)=\operatorname{dist}_{X_{Q}}\left(c_{2}(w), E\right)
$$

for $w \in C_{Q}$ in some neighborhood $U_{2} \subset C_{Q}$ of $F_{Q}^{0}(e)$. The desired neighborhood is

$$
V=U_{1} \cap U_{2} \subset C_{Q}
$$

By Proposition 10.4 the restricted correspondence $\overline{C_{Q}} \rightrightarrows \bar{X}_{Q}$ is neutral near $F_{Q}(e)$. So if $\mathbf{P} \subset \mathbf{Q}$ and if $F_{Q}(e) \cap C^{P} \neq \emptyset$ and if $\Delta_{P}=i\left(\Delta_{Q}\right) \amalg J$ as in (2.4.1) then by (10.4.1),

$$
r_{\alpha}^{P}\left(c_{2}(w)\right)=r_{\alpha}^{P}\left(c_{1}(w)\right)
$$

for all $w \in C_{Q}^{P}$ and for all $\alpha \in J$. Moreover, by Lemma 10.7 the correspondence preserves tiles near $F_{Q}(e)$, that is, for all $w \in U_{1}$ and for all $\mathbf{P} \subseteq \mathbf{Q}$ we have 


$$
w \in \bar{C}_{Q}^{P} \Leftrightarrow c_{1}(w) \in \bar{X}_{Q}^{P} \Leftrightarrow c_{2}(w) \in \bar{X}_{Q}^{P}
$$

By (7.1.3) the correspondence commutes with the geodesic action of $A_{P}$. Therefore

$$
R_{X_{Q}}\left(c_{1}(w)\right)=c_{1}\left(R_{C_{Q}}(w)\right) \quad \text { and } \quad R_{X_{Q}}\left(c_{2}(w)\right)=c_{2}\left(R_{C_{Q}}(w)\right)
$$

Now suppose $w \in V$ and $w \in C_{Q}^{P}$ for some $\mathbf{P} \subseteq \mathbf{Q}$. If $\mathbf{P}=\mathbf{Q}$ (that is, if $w \in C_{Q}^{0}$ lies in the central tile) then $c_{i}(w) \in C_{Q}^{0}$ as well, in which case $R_{X_{Q}}\left(c_{i}(w)\right)=c_{i}(w), W_{Q}\left(c_{i}(w)\right)=0$, and $c_{i}(w)=c_{i}^{\prime}(w)$. Then (11.5.1) follows from (11.6.1) and in fact, equality holds.

Now suppose $w \in V \cap C_{Q}^{P}$ for some $\mathbf{P} \neq \mathbf{Q}$. Then $\mathbf{P} \subset \mathbf{Q}, c_{1}\left(C_{P}\right)=c_{2}\left(C_{P}\right)=X_{P}$ by Proposition 7.3, and locally near $X_{P}$ this correspondence is isomorphic to a parabolic Hecke correspondence, that is, we may assume that $g \in \mathbf{P}(\mathbb{Q})$. In the tile $C^{P}$ the retraction $R$ commutes with the geodesic action of $A_{P}$, cf. (4.6.1), and so does the Hecke correspondence, (7.1.3), hence

$$
R_{X_{Q}}\left(c_{2}^{\prime}(w)\right)=R_{X_{Q}}\left(\operatorname{Sh}(\mathbf{t}) c_{2}(w)\right)=R_{X_{Q}}\left(c_{2}(w)\right)=c_{2}\left(R_{C_{Q}}(w)\right)
$$

So the second terms in (11.4.1) are equal:

$$
\begin{aligned}
\operatorname{dist}_{X_{Q}}\left(R_{X_{Q}} c_{2}^{\prime}(w), E\right) & =\operatorname{dist}_{X_{Q}}\left(c_{2}\left(R_{C_{Q}}(w)\right), c_{2}\left(F_{Q}^{0}(e)\right)\right)=\operatorname{dist}_{C_{Q}}\left(R_{C_{Q}}(w), F_{Q}^{0}((e))\right) \\
& =\operatorname{dist}_{X_{Q}}\left(c_{1}\left(R_{C_{Q}}(w)\right), E\right)=\operatorname{dist}_{X_{Q}}\left(R_{X_{Q}} c_{1}(w), E\right)
\end{aligned}
$$

because both morphisms $c_{1}$ and $c_{2}$ are local isometries. Now consider the first terms in (11.4.1). Fix $w \in C_{Q}^{P}$. For $\alpha \in \Delta_{P}$ set $\rho(\alpha)=\rho\left(r_{\alpha}^{P}\left(c_{2}(w)\right)\right)$. Using (4.6.3), (5.5.1), and (11.6.2) we find,

$$
\begin{aligned}
W_{Q}\left(c_{2}^{\prime}(w)\right) & =1-\inf _{\alpha \in J}\left\{r_{\alpha}^{P} \operatorname{Sh}(\mathbf{t}) c_{2}(w)\right\} \\
& =1-\inf _{\alpha \in J}\left\{r_{\alpha}^{P}\left(c_{2}(w)\right) \alpha_{0}(\mathbf{t})^{-\rho(\alpha)}\right\} \\
& =1-\inf _{\alpha \in J}\left\{r_{\alpha}^{P}\left(c_{1}(w)\right) \alpha_{0}(\mathbf{t})^{-\rho(\alpha)}\right\} \\
& \geqq 1-\inf _{\alpha \in J}\left\{r_{\alpha}^{P}\left(c_{1}(w)\right)\right\} \\
& =W_{Q}\left(c_{1}^{\prime}(w)\right)
\end{aligned}
$$

which completes the proof of (11.5.1).

11.7. Hyperbolic correspondences. Recall that the correspondence $\left(c_{1}, c_{2}\right): \bar{C} \rightrightarrows \bar{X}$ is weakly hyperbolic ([GM2], [GM5]) near a connected component $F \subset \bar{C}$ of the fixed point set, if there is a neighborhood $N\left(F^{\prime}\right) \subset \bar{X}$ of the image $F^{\prime}=c_{1}(F)=c_{2}(F)$ and an indicator mapping $t=\left(t_{1}, t_{2}\right): N\left(F^{\prime}\right) \rightarrow \mathbb{R}_{\geqq 0} \times \mathbb{R}_{\geqq 0}$ such that

(1) the mapping $t$ is proper and subanalytic;

(2) the pre-image of the origin $t^{-1}(0)=F^{\prime}$ consists precisely of $F^{\prime}$; 
(3) there is a neighborhood $N(F) \subset \bar{C}$ so that $c_{i}(N(F)) \subset N\left(F^{\prime}\right)($ for $i=1,2)$ and

$$
N(F) \cap c_{1}^{-1}\left(F^{\prime}\right) \cap c_{2}^{-1}\left(F^{\prime}\right)=F
$$

(4) for any $x \in N(F)$,

$$
\begin{aligned}
& t_{1}\left(c_{1}(x)\right) \leqq t_{1}\left(c_{2}(x)\right), \\
& t_{2}\left(c_{1}(x)\right) \geqq t_{2}\left(c_{2}(x)\right) .
\end{aligned}
$$

(Due to an error in [GM2], condition (3) above was omitted from the original definition of weakly hyperbolic, cf. [GM5].)

11.8. The modified correspondence is hyperbolic. Choose a tiling parameter $\mathbf{b} \in \mathscr{B}$ so that the associated tiling is narrow with respect to the Hecke correspondence $\left(c_{1}, c_{2}\right): \bar{C} \rightrightarrows \bar{X}$. Choose $\mathbf{t} \in A_{P_{0}}(>1)$ to be dominant, regular, and sufficiently close to $\mathbf{1}$ as in Proposition 11.2 and equation (11.3.1). Let $\left(c_{1}^{\prime}, c_{2}^{\prime}\right): \bar{C} \rightrightarrows \bar{X}$ be the modified correspondence.

Suppose $C_{Q}$ is a stratum of $\bar{C}$ for which $F \cap C_{Q} \neq \emptyset$. Then $c_{1}\left(C_{Q}\right)=c_{2}\left(C_{Q}\right)=X_{Q}$. By Proposition 7.3 we may, locally near $C_{Q}$, replace the Hecke correspondence with a parabolic Hecke correspondence determined by some $g=u_{g} a_{g} m_{g} \in \mathbf{Q}(\mathbb{Q})$. For any $e \in L_{Q}$ let $F_{Q}(e) \subset C_{Q}$ denote the corresponding fixed point constituent: the set of fixed points in $C_{Q}$ for which $e$ is a characteristic element as in $§ 8.2$. By Proposition 11.2 the fixed point set in $C_{Q}$ of the modified Hecke correspondence is a union of "truncated" constituents $F_{Q}^{0}(e)=F_{Q}(e) \cap C_{Q}^{0}$ (as $e$ varies over elliptic elements in $\Gamma_{L} \bar{g} \Gamma_{L}$, cf. Proposition 8.4). (Although $F_{Q}^{0}(e)$ may have finitely many connected components we will treat them all simultaneously.)

Fix such an element $e$ and let $E=c_{1}\left(F_{Q}(e)\right) \cap X_{Q}^{0}=c_{1}\left(F_{Q}^{0}(e)\right)$ be the image in $X_{Q}$ of the truncated fixed point constituent as in $\S 11.4$. Write $\Delta_{Q}=\Delta_{Q}^{+} \cup \Delta_{Q}^{-} \cup \Delta_{Q}^{0}$ according to whether the simple root is expanding, contracting, or neutral near $X_{Q}$ as in $\S 7.1$. Define $t=\left(t_{1}, t_{2}\right): T\left(X_{Q}\right) \rightarrow \mathbb{R}_{\geqq 0} \times \mathbb{R}_{\geqq 0}$ by

$$
\begin{aligned}
& t_{1}(x)=\sum_{\alpha \in \Delta_{Q}^{+}} r_{\alpha}^{Q}(x)+d_{E}\left(\pi_{Q}(x)\right), \\
& t_{2}(x)=\sum_{\alpha \in \Delta_{Q}^{-}} r_{\alpha}^{Q}(x)+\sum_{\alpha \in \Delta_{Q}^{0}} r_{\alpha}^{Q}(x) .
\end{aligned}
$$

Here,

$$
T\left(X_{Q}\right)=T\left(\bar{X}_{Q}\right) \cap X[Q]
$$

denotes the open neighborhood of $X_{Q}$ on which the above mappings are defined: it consists of the part of $T\left(\bar{X}_{Q}\right)$ which is contained in those strata $X_{P}$ such that $\mathbf{Q} \subseteq \mathbf{P}$.

11.9. Theorem. The mapping $\left(t_{1}, t_{2}\right)$ is an indicator mapping, with respect to which the modified Hecke correspondence is hyperbolic near $F_{Q}^{0}(e)$. 
11.10. Proof. The idea is that the composition with the $\operatorname{Sh}(\mathbf{t})$ converts neutral directions (normal to a given stratum) into contracting directions but it does not change the nature of the expanding or contracting (normal) directions. It converts distances within the stratum (which are preserved by the Hecke correspondence and hence neutral) into expanding directions.

We must display a neighborhood $N\left(F_{Q}^{0}(e)\right) \subset \bar{C}$ which satisfies conditions (3) and (4) of $\$ 11.7$ (but with $F$ replaced by $F_{Q}^{0}(e)$ ). First we find a neighborhood $N_{1}$ so that condition (3) holds. Since $c_{1}: \bar{C} \rightarrow \bar{X}$ is stratum preserving,

$$
c_{1}^{-1}(E) \cap T\left(C_{Q}\right)=c_{1}^{-1}(E) \cap C_{Q}
$$

where $T\left(C_{Q}\right)=T\left(\bar{C}_{Q}\right) \cap C[Q]$. The mapping $c_{1}: C_{Q} \rightarrow X_{Q}$ is a finite (unramified) covering. Therefore, if $W \subset C_{Q}$ is a sufficiently small neighborhood of $F_{Q}^{0}(e)$ in $C_{Q}$ then

$$
c_{1}^{-1}(E) \cap W=F_{Q}^{0}(e) .
$$

Take $N_{1}=\pi_{Q}^{-1}(W) \cap T\left(C_{Q}\right)$. This neighborhood of $F_{Q}^{0}(e)$ satisfies condition (3) because

$$
c_{1}^{-1}(E) \cap c_{2}^{-1}(E) \cap N_{1} \subset c_{1}^{-1}(E) \cap N_{1}=c_{1}^{-1}(E) \cap W=F_{Q}^{0}(e)
$$

and the reverse inclusion is obvious.

Now consider the conditions (4). Let $a_{Q}=a_{g} \in A_{Q}$ be the torus factor for the correspondence near $C_{Q}$. It is easy to check that $t_{1}^{-1}(0) \cap t_{2}^{-1}(0)=E$. For any $w \in T\left(\bar{C}_{Q}\right)$ and for all $\alpha \in \Delta_{Q}$ we have

$$
\begin{aligned}
r_{\alpha}^{Q}\left(c_{2}^{\prime}(w)\right) & =r_{\alpha}^{Q}\left(\operatorname{Sh}(\mathbf{t}) c_{2}(w)\right)=\alpha_{0}(\mathbf{t})^{-\rho\left(r_{\alpha}^{Q} c_{2}(w)\right)} r_{\alpha}^{Q} c_{2}(w) & & \text { by }(5.5 .1) \\
& =\alpha_{0}(\mathbf{t})^{-\rho\left(r_{\alpha}^{Q} c_{2}(w)\right)} \alpha\left(a_{Q}\right)^{-1} r_{\alpha}^{Q}\left(c_{1}(w)\right) & & \text { by }(10.4 .1) .
\end{aligned}
$$

If $\alpha \in \Delta_{Q}^{-} \cup \Delta_{Q}^{0}$ then $\alpha_{0}(\mathbf{t})^{-\rho\left(r_{\alpha}^{Q} c_{2}(w)\right)} \alpha\left(a_{Q}\right)^{-1} \leqq 1$ since both factors are $\leqq 1$. This proves that

$$
t_{2} c_{2}^{\prime}(w) \leqq t_{2} c_{1}^{\prime}(w)
$$

If $\alpha \in \Delta_{Q}^{+}$then $\alpha\left(a_{Q}\right)<1$ so $\alpha_{0}(\mathbf{t})^{-\rho\left(r_{\alpha}^{Q} c_{2}(w)\right)} \alpha\left(a_{Q}\right)^{-1}>1$ by (11.3.1). Let $V \subset C_{Q}$ be the neighborhood of $F_{Q}^{0}(e)$ described in Lemma 11.5. Then for all $w \in \pi_{Q}^{-1}(V)$ we have

$$
\begin{array}{rlr}
d_{E} \pi_{Q} c_{2}^{\prime}(w) & =d_{E} \pi_{Q} \operatorname{Sh}(\mathbf{t}) c_{2}(w) & \\
& =d_{E} \operatorname{Sh}(\mathbf{t}) \pi_{Q} c_{2}(w) & \text { by } \S 5.5(4) \\
& =d_{E} \operatorname{Sh}(\mathbf{t}) c_{2} \pi_{Q}(w) \quad \text { by }(6.4 .3) \\
& \geqq d_{E} c_{1}^{\prime} \pi_{Q}(w) & \text { by }(11.5 .1) \\
& =d_{E} \pi_{Q} c_{1}^{\prime}(w) &
\end{array}
$$

which proves that 


$$
t_{1} c_{2}^{\prime}(w) \geqq t_{1} c_{1}^{\prime}(w)
$$

for all $w \in \pi_{Q}^{-1}(V)$. This completes the verification of condition (4) of $\$ 11.7$. In summary, the neighborhood

$$
N\left(F_{Q}^{0}(e)\right)=N_{1} \cap \pi_{Q}^{-1}(V) \subset \bar{C}
$$

satisfies both conditions (3) and (4).

\section{Local weighted cohomology with supports}

12.1. Quadrants (See $[\mathrm{GKM}], \S 7.14$, p. 534). As in previous sections we suppose $\mathbf{G}$ is a connected reductive linear algebraic group defined over $\mathbb{Q}$ and we denote the greatest $\mathbb{Q}$-split torus in its center by $\mathbf{S}_{\mathbf{G}}$. Let $\mathbf{P}$ be a rational parabolic subgroup with $\mathbf{S}_{\mathbf{P}}$ the greatest $\mathbb{Q}$-split torus in the center of its Levi quotient $\mathbf{L}_{\mathbf{P}}$. Let $\Delta_{P}$ denote the simple positive roots of $\mathbf{S}_{\mathbf{P}}$ occurring in $\mathfrak{N}_{P}=\operatorname{Lie}\left(\mathscr{U}_{P}\right)$. The elements $\alpha \in \Delta_{P}$ are trivial on $\mathbf{S}_{\mathbf{G}}$ and form a basis of $\chi_{\mathbb{Q}}^{*}\left(\mathbf{S}_{\mathbf{P}}^{\prime}\right) \subset \chi_{\mathbb{Q}}^{*}\left(\mathbf{S}_{\mathbf{P}}\right)$ where $\mathbf{S}_{\mathbf{P}}^{\prime}=\mathbf{S}_{\mathbf{P}} / \mathbf{S}_{\mathbf{G}}$. For any subset $J \subset \Delta_{P}$ as in $\S 2.4$ let $\mathbf{Q}=\mathbf{P}(\mathbf{J})$ be the parabolic subgroup containing $\mathbf{P}$ for which the corresponding torus

$$
\mathbf{S}_{\mathbf{J}}=\mathbf{S}_{\mathbf{P}(\mathbf{J})}
$$

is the identity component of the intersection $\bigcap_{\alpha \in J} \operatorname{ker}(\alpha)$. Let $\left\{t_{\alpha}\right\}$ be the basis of the cocharacter group $\chi_{*}\left(\mathbf{S}_{\mathbf{P}}^{\prime}\right) \otimes \mathbb{Q}$ which is dual to the basis $\Delta_{P}$ so that $\left\langle\alpha, t_{\beta}\right\rangle=\delta_{\alpha, \beta}$ (with respect to the canonical pairing $\langle\cdot, \cdot\rangle)$. The cocharacter group $\chi_{*}^{\mathbb{Q}}\left(\mathbf{S}_{\mathbf{P}} / \mathbf{S}_{\mathbf{J}}\right)$ is spanned by $\left\{t_{\alpha} \mid \alpha \in J\right\}$ while $\chi_{*}^{\mathbb{Q}}\left(\mathbf{S}_{\mathbf{J}} / \mathbf{S}_{\mathbf{G}}\right)$ is spanned by $\left\{t_{\alpha} \mid \alpha \in \bar{J}\right\}$, where $\bar{J}=\Delta_{P}-J$ denotes the complement.

Fix $v_{P} \in \chi_{\mathbb{Q}}^{*}\left(\mathbf{S}_{\mathbf{P}}\right)$ and $J \subset \Delta_{P}$. Let $\gamma \in \chi_{\mathbb{Q}}^{*}\left(\mathbf{S}_{\mathbf{P}}\right)$ and suppose that $\gamma\left|\mathbf{S}_{\mathbf{G}}=v_{P}\right| \mathbf{S}_{\mathbf{G}}$. Then $\gamma-v_{P}$ may be regarded as an element of $\chi_{\mathbb{Q}}^{*}\left(\mathbf{S}_{\mathbf{P}}^{\prime}\right)$ so we may define

$$
\begin{aligned}
I_{v_{P}}(\gamma) & =\left\{\alpha \in \Delta_{P} \mid\left\langle\gamma-v_{P}, t_{\alpha}\right\rangle<0\right\}, \\
\chi_{\mathbb{Q}}^{*}\left(\mathbf{S}_{\mathbf{P}}\right)_{\left\lceil v_{P}, J\right\rfloor} & =\left\{\gamma \in \chi_{\mathbb{Q}}^{*}\left(\mathbf{S}_{\mathbf{P}}\right) \mid I_{v_{P}}(\gamma)=J \text { and } \gamma\left|\mathbf{S}_{\mathbf{G}}=v_{P}\right| \mathbf{S}_{\mathbf{G}}\right\} .
\end{aligned}
$$

This last set is called the quadrant of type $J$. The disjoint union of the $2^{\left|\Delta_{P}\right|}$ quadrants,

$$
\coprod_{J \cong \Delta_{P}} \chi_{\mathbb{Q}}^{*}\left(\mathbf{S}_{\mathbf{P}}\right)_{\left\lceil v_{P}, J\right\rfloor}=\left\{\gamma \in \chi_{\mathbb{Q}}^{*}\left(\mathbf{S}_{\mathbf{P}}\right)|\gamma| \mathbf{S}_{\mathbf{G}}=v_{P} \mid \mathbf{S}_{\mathbf{G}}\right\}
$$

is the subset of all characters whose restriction to $\mathbf{S}_{\mathbf{G}}$ agrees with that of $v_{P}$. Taking $J=\emptyset$ gives

$$
\chi_{\mathbb{Q}}^{*}\left(\mathbf{S}_{\mathbf{P}}\right)_{\left\lceil v_{P}, \phi\right\rfloor}=\left\{\gamma \in \chi_{\mathbb{Q}}^{*}\left(\mathbf{S}_{\mathbf{P}}\right)|\gamma| \mathbf{S}_{\mathbf{G}}=v_{P} \mid \mathbf{S}_{\mathbf{G}} \text { and }\left\langle\gamma-v_{P}, t_{\alpha}\right\rangle \geqq 0 \text { for all } \alpha \in \Delta_{P}\right\}
$$

which was denoted $\chi_{\mathbb{Q}}^{*}\left(\mathbf{S}_{\mathbf{P}}\right)_{+}$in $[\mathrm{GHM}]$ and was denoted $\chi_{\mathbb{Q}}^{*}\left(\mathbf{S}_{\mathbf{P}}\right)_{\geqq v_{P}}$ in $[\mathrm{GKM}]$. It is the translate by $v_{P}$ of the positive cone $\left\{\sum_{\alpha \in \Delta_{P}} m_{\alpha} \alpha\right\}$ with $m_{\alpha} \geqq 0$. More generally, for $J \subset \Delta_{P}$ define $\chi_{\mathbb{Q}}^{*}\left(\mathbf{S}_{\mathbf{P}}\right)_{\geqq v_{P}(J)}$ to be the translate by $v_{P}$ of the cone $\left\{\sum_{\alpha \in \bar{J}} m_{\alpha} \alpha \mid m_{\alpha} \geqq 0\right\}$. That is, 


$$
\begin{aligned}
\chi_{\mathbb{Q}}^{*}\left(\mathbf{S}_{\mathbf{P}}\right)_{\geqq v_{P}(J)} & =\left\{\gamma \in \chi_{\mathbb{Q}}^{*}\left(\mathbf{S}_{\mathbf{P}}\right)|\gamma| \mathbf{S}_{\mathbf{G}}=v_{P} \mid \mathbf{S}_{\mathbf{G}} \text { and }\left\langle\gamma-v_{P}, t_{\alpha}\right\rangle \geqq 0 \text { for all } \alpha \in \bar{J}\right\} \\
& =\bigcup_{K \cong J} \chi_{\mathbb{Q}}^{*}\left(\mathbf{S}_{\mathbf{P}}\right)_{\left\lceil v_{P}, K\right\rfloor} .
\end{aligned}
$$

Then

$$
\chi_{\mathbb{Q}}^{*}\left(\mathbf{S}_{\mathbf{P}}\right)_{\left\lceil v_{P}, J\right\rfloor}=\chi_{\mathbb{Q}}^{*}\left(\mathbf{S}_{\mathbf{P}}\right)_{\geqq v_{P}(J)}-\bigcup_{K \subsetneq J} \chi_{\mathbb{Q}}^{*}\left(\mathbf{S}_{\mathbf{P}}\right)_{\geqq v_{P}(K)}
$$

Equation (12.1.3) remains valid if we replace the union on the right hand side by the union over those $K \subset J$ such that $|K|=|J|-1$.

(This apparently backward notation was chosen so as to simplify the computation in $\S 12.6$. It can be reconciled with the notation of $[\mathrm{GHM}]$ as follows. There are $\left|\Delta_{P}\right|$ proper maximal parabolic subgroups containing P. Each $J \subset \Delta_{P}$ corresponds to a collection $\hat{J}$ of these maximal parabolic subgroups, with $\mathbf{Q} \in \hat{J}$ iff $\mathbf{S}_{\mathbf{Q}} \subset \bigcap_{\alpha \in J} \operatorname{ker}(\alpha)$. Then the subset $\chi_{\mathbb{Q}}^{*}\left(\mathbf{S}_{\mathbf{P}}\right)_{\geqq v_{P}(J)}$ in this paper coincides with the subset $\chi_{\mathbb{Q}}^{*}\left(\mathbf{S}_{\mathbf{P}}\right)_{+(\hat{J})}$ in $[\mathrm{GHM}]$.)

If $H$ is an $\mathbf{S}_{\mathbf{P}}$ module such that $\mathbf{S}_{\mathbf{G}}$ acts on $H$ through the character $v_{P} \mid \mathbf{S}_{\mathbf{G}}$ then one may define $H_{\left\lceil v_{P}, J\right\rfloor}$ (resp. $H_{\geqq v_{P}}$, resp. $\left.H_{\geqq v_{P}(J)}\right)$ to be the sum of those weight spaces $H_{\gamma}$ for which $\gamma \in \chi_{\mathbb{Q}}^{*}\left(\mathbf{S}_{\mathbf{P}}\right)_{\left\lceil v_{P}, J\right\rfloor}\left(\right.$ resp. $\gamma \in \chi_{\mathbb{Q}}^{*}\left(\mathbf{S}_{\mathbf{P}}\right)_{\geqq v_{P}}$, resp. $\left.\gamma \in \chi_{\mathbb{Q}}^{*}\left(\mathbf{S}_{\mathbf{P}}\right)_{\geqq v_{P}(J)}\right)$.

12.2. Weighted cohomology. As in $\S 2$, let $D$ denote the symmetric space associated to $G, K^{\prime}=A_{G} K\left(x_{0}\right)$ denote the stabilizer in $G$ of a fixed basepoint $x_{0} \in D, \Gamma \subset \mathbf{G}(\mathbb{Q})$ be a neat arithmetic group and $X=\Gamma \backslash D$. Let $G \rightarrow G L(E)$ be a finite dimensional irreducible representation of $\mathbf{G}$ on some complex vector space $E$. It gives rise to a local system $\mathbf{E}=\left(G / K^{\prime}\right) \times_{\Gamma} E$ on $X=\Gamma \backslash G / K$. Let $\mathbf{P}_{\mathbf{0}}$ be the standard minimal rational parabolic subgroup with $\mathbf{S}_{\mathbf{0}}=\mathbf{S}_{\mathbf{P}_{\mathbf{0}}}$. Fix $v \in \chi_{\mathbb{Q}}^{*}\left(\mathbf{S}_{\mathbf{0}}\right)$ so that $v \mid \mathbf{S}_{\mathbf{G}}$ coincides with the character by which $\mathbf{S}_{\mathbf{G}}$ acts on $E$. Then $v$ defines a weight profile in the sense of $[\mathrm{GHM}]:$ if $\mathbf{Q} \supseteqq \mathbf{P}_{\mathbf{0}}$ is a standard rational parabolic subgroup then set $v_{Q}=v \mid \mathbf{S}_{\mathbf{Q}}$ and

$$
\chi_{\mathbb{Q}}^{*}\left(\mathbf{S}_{\mathbf{Q}}\right)_{+}=\chi_{\mathbb{Q}}^{*}\left(\mathbf{S}_{\mathbf{Q}}\right)_{\geqq v_{Q}}=\chi_{\mathbb{Q}}^{*}\left(\mathbf{S}_{\mathbf{Q}}\right)_{\left\lceil v_{Q}, \phi\right\rfloor}
$$

These definitions may be extended to arbitrary rational parabolic subgroups by conjugation. We obtain from $[\mathrm{GHM}]$ a complex of fine sheaves, $\mathbf{W}^{v} \mathbf{C}^{\bullet}(\mathbf{E})$ on the reductive BorelSerre compactification $\bar{X}$ of $X$, whose (hyper)-cohomology groups $W^{v} H^{*}(\bar{X}, \mathbf{E})$ are the weighted cohomology groups. Let $i: X \rightarrow \bar{X}$ denote the inclusion. Recall from [GHM], $\S 13$ that a choice of basepoint induces an isomorphism

$$
\mathbf{H}_{x}^{j}\left(R i_{*}(\mathbf{E})\right) \cong H^{j}\left(\mathfrak{M}_{Q}, E\right)
$$

between the stalk cohomology at a point $x \in X_{Q}$ of the complex of sheaves $R i_{*}(\mathbf{E})$ and the Lie algebra cohomology of $\mathfrak{N}_{Q}$. The weighted cohomology complex is obtained by applying a weight truncation to the complex $R i_{*}(\mathbf{E})$ with the result that its stalk cohomology becomes

$$
\mathbf{H}_{x}^{j}\left(\mathbf{W}^{v} \mathbf{C}^{\bullet}(\mathbf{E})\right)=H^{j}\left(\mathfrak{P}_{Q}, E\right)_{\geqq v_{Q}} .
$$


12.3. Remarks on sheaf theory. In the next few sections we will need to use the formalism of the derived category of sheaves, and some relations between the standard functors, for which we refer to [GM4], [GM2], [Bo5], [I], [KS], [GeM]. Specifically, if $X$ is a subanalytic set we denote by $D^{b}(X)$ the bounded (cohomologically-) constructible derived category of sheaves of complex vector spaces on $X$. An element $\mathbf{S}^{\bullet} \in D^{b}(X)$ is a complex of sheaves, bounded from below, whose cohomology sheaves $\mathbf{H}^{i}\left(\mathbf{S}^{\bullet}\right)$ are finite dimensional and are locally constant on each stratum of some subanalytic stratification of $X$. The hypercohomology of $\mathbf{S}^{\bullet}$ will be denoted $H^{*}\left(\mathbf{S}^{\bullet}\right)$ and the stalk cohomology at a point $x \in X$ will be denoted $H_{x}^{*}\left(\mathbf{S}^{\bullet}\right)$. Denote by $\mathbf{S}[n]^{\bullet}$ the shifted sheaf, $\mathbf{S}[n]^{p}=\mathbf{S}^{n+p}$. The derived category $D^{b}(X)$ supports the standard operations of $R \mathbf{H o m}, \otimes, R f_{*}, R f_{!}, f^{*}$, and $f^{!}$. There are many relations between these functors, of which we mention a few that we will use:

If $f: Y \rightarrow X$ is a normally nonsingular embedding ([GM4], §5.4) then there is a canonical isomorphism

$$
f^{!}\left(\mathbf{S}^{\bullet}\right) \cong f^{*}\left(\mathbf{S}^{\bullet}\right) \otimes \mathcal{O}_{X / Y}[-d]
$$

where $\mathcal{O}_{X / Y}$ denotes the orientation bundle (or top exterior power) of the normal bundle of $Y$ in $X$, and where $d$ denotes the codimension of $Y$ in $X$. If $f: X \rightarrow$ pt is the map to a point then $\mathbb{D}_{X}=f^{!}(\mathbb{C})$ is the dualizing complex. If $X$ is an $n$-dimensional manifold (or even a rational homology manifold) then $\mathbb{D}_{X} \cong \mathcal{O}_{X}[n]$ where $\mathcal{O}_{X}$ denotes the orientation bundle.

12.4. Cohomology with supports. Let $\bar{X}$ be a compact subanalytic set and let $\mathbf{S}^{\bullet}$ be a (cohomologically) constructible complex of sheaves on $\bar{X}$. Suppose $Y \subset W \subset \bar{X}$ are locally closed subsets with inclusions

$$
Y \underset{h_{Y}}{\rightarrow} W \underset{j_{W}}{\rightarrow} \bar{X}
$$

Define the restriction of $\mathbf{S}^{\bullet}$ to $Y$ with compact supports in $W$ to be the complex of sheaves

$$
\mathbf{B}^{\bullet}=h_{Y}^{!} j_{W}^{*} \mathbf{S}^{\bullet}
$$

If $Y=\{y\}$ is a single point, then the cohomology of this complex is the relative cohomology group

$$
H^{m}\left(\mathbf{B}^{\bullet}\right)=H^{m}\left(B_{\varepsilon} \cap \bar{X}, \partial B_{\varepsilon} \cap W ; \mathbf{S}^{\bullet}\right),
$$

where $B_{\varepsilon}$ is a sufficiently small ball around $y$ (with respect to some subanalytic embedding in Euclidean space) and $\partial B_{\varepsilon}$ is its boundary.

Now suppose $\bar{X}$ is the reductive Borel-Serre compactification of a locally symmetric space $X=\Gamma \backslash G / K$ as in $\S 12.2$, and that $\mathbf{S}^{\bullet}=\mathbf{W}^{v} \mathbf{C}^{\bullet}(\mathbf{E})$ is the weighted cohomology sheaf constructed with respect to some weight profile $v$ and local system $\mathbf{E}$ as in $\$ 12.2$. Let $Y=X_{P}$ be some stratum and let $W=\bar{X}_{Q}$ be the closure of a larger stratum, corresponding to some rational parabolic subgroup $\mathbf{Q} \supset \mathbf{P}$. Form $\mathbf{B}^{\bullet}=h_{Y}^{!} j_{W}^{*} \mathbf{W}^{v} \mathbf{C}^{\bullet}(\mathbf{E})$ as above. Write $\Delta_{P}=i\left(\Delta_{Q}\right) \amalg I$ as in $(2.4 .1)$. 
12.5. Theorem. The cohomology sheaf $\mathbf{H}^{m}\left(\mathbf{B}^{\bullet}\right)$ is isomorphic to the local system on $X_{P}$ which is associated to the following $L_{P}$-submodule of the $\mathfrak{N}_{P}$-cohomology,

$$
H^{m-|I|}\left(\mathfrak{M}_{P}, E\right)_{\left\lceil v_{P}, I\right\rfloor} \cdot
$$

12.6. Proof of Theorem 12.5. The proof follows closely the computation [GHM], $\S 18$ of the weighted cohomology of the link $\mathscr{L}_{y}$. First let us recall some generalities. Each stratum $X_{P}$ of the reductive Borel-Serre compactification $\bar{X}$ is a rational homology manifold. If $\Gamma$ is neat, then each stratum is a smooth manifold. Suppose $\mathbf{S}^{\bullet}$ is a complex of sheaves whose cohomology sheaves are locally constant on each stratum of $\bar{X}$. Let $Y=X_{P} \subset W=\bar{X}_{Q}$ as above. The choice of basepoint $x_{0} \in D$ determines a basepoint $y \in Y$. Let $N_{y} \subset \bar{X}$ be a normal slice (cf. [GM3], \$5.4) to the stratum $Y$ at the point $y$. Let $k: N_{y} \cap W \rightarrow \bar{X}$ denote the inclusion, and let $i_{y}$ and $a_{y}$ denote the inclusions of $y$ into $Y$ and $N_{y} \cap W$ respectively.

Then the stalk cohomology of $\mathbf{B}^{\bullet}=h_{Y}^{!} j_{W}^{*} \mathbf{S}^{\bullet}$ is given by

$$
H_{y}^{m}\left(\mathbf{B}^{\bullet}\right)=H^{m}\left(i_{y}^{*} h_{Y}^{!} j_{W}^{*} \mathbf{S}^{\bullet}\right) \cong H^{m}\left(a_{y}^{!} k^{*} \mathbf{S}^{\bullet}\right)
$$

which in turn may be identified with the relative cohomology group

$$
H^{m}\left(B_{\varepsilon} \cap N_{y}, \partial B_{\varepsilon} \cap N_{y} \cap \bar{X}_{Q} ; \mathbf{S}^{\bullet}\right)
$$

(where $B_{\varepsilon}$ is a sufficiently small ball around $y$, chosen with respect to some locally defined subanalytic embedding of $\bar{X}$ into some Euclidean space).

These isomorphisms are deduced from the following fiber squares

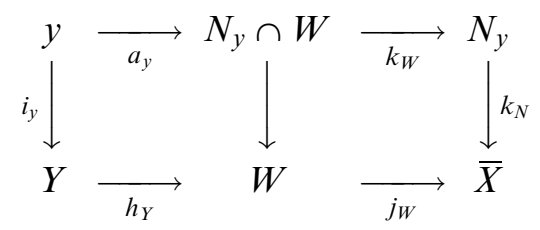

where $k=k_{N} k_{W}$. In the case that $\mathbf{S}^{\bullet}=\mathbf{W}^{v} \mathbf{C}^{\bullet}$ we will compute (12.6.2) using the long exact cohomology sequence for the pair.

Step 1. Construct an isomorphism of $L_{P}$-modules,

$$
H^{c}\left(\partial B_{\varepsilon} \cap N_{y} \cap \bar{X}_{Q} ; \mathbf{W}^{v} \mathbf{C}^{\bullet}(\mathbf{E})\right) \cong H^{c-|I|+1}\left(\mathfrak{N}_{P}, E\right)_{\left\lceil v_{P}, I\right\rfloor} \oplus H^{c}\left(\mathfrak{N}_{P}, E\right)_{\geqq v_{P}}
$$

In order to simplify notation, let us choose a labeling $\left\{\alpha_{1}, \alpha_{2}, \ldots, \alpha_{s}\right\}=\Delta_{P}$ of the simple roots. As in $[\mathrm{GHM}], \S 8.8$, the link $\mathscr{L}_{y}=\partial B_{\varepsilon} \cap N_{y}$ comes with a natural mapping $\delta: \mathscr{L}_{y} \rightarrow \triangleright^{s-1}$ to the $s-1$ dimensional simplex,

$$
\triangleright^{s-1}=\left\{\left(x_{1}, x_{2}, \ldots, x_{s}\right) \in \mathbb{R}^{s} \mid 0 \leqq x_{i} \leqq 1 \text { and } \sum_{i=1}^{s} x_{i}=1\right\}
$$


For any subset $J \subset\{1,2, \ldots, s\}$ let $\bar{J}$ denote its complement. Associated to $J$ there is a (closed) face of dimension $|J|-1$,

$$
\triangleright_{J}=\left\{x \in \triangleright^{s-1} \mid x_{j}=0 \text { for all } j \in \bar{J}\right\}
$$

whose interior we denote by $\triangleright_{J}^{o}$. Each $\triangleright_{\{j\}}$ is a vertex of $\triangleright^{s-1}$; the face $\triangleright_{J}$ is spanned by the vertices $\triangleright_{\{j\}}$ such that $j \in J$. Let $U_{\{j\}}=S t\left(\triangleright_{\{j\}}\right)$ be the open star of the vertex $\triangleright_{\{j\}}$. These form a covering of $\triangleright^{s-1}$ whose multi-intersections we denote by

$$
U_{J}=\bigcap_{j \in J} U_{\{j\}}
$$

Then

$$
U_{J}=S t\left(\triangleright_{J}^{o}\right)=\bigcup\left\{F^{o} \mid F \text { is a face of } \triangleright^{s-1} \text { and } F \supseteqq \triangleright_{J}\right\}
$$

is the open star of the interior of the face $\triangleright_{J}$.

If we think of stratifying the simplex $\triangleright^{s-1}$ by the interiors of its faces, then the mapping $\delta: \mathscr{L}_{y} \rightarrow \triangleright^{s-1}$ is a stratified mapping: for any $J \subset\{1,2, \ldots, s\}$ it maps $\mathscr{L}_{y} \cap X_{P(J)}$ to the interior $\triangleright_{J}^{o}$ of the face $\triangleright_{J}$, and in particular

$$
\mathscr{L}_{y} \cap \bar{X}_{Q}=\delta^{-1}\left(\triangleright_{I}\right)
$$

The fiber over any interior point $s \in \triangleright_{I}^{o}$ is the nilmanifold $\left(\Gamma \cap \mathscr{U}_{Q}\right) \backslash \mathscr{U}_{Q}$. As in $[\mathrm{GHM}]$, $\S 18.5$, the (weighted) cohomology of $\mathscr{L}_{y} \cap \bar{X}_{Q}$ can be computed using the Mayer-Vietoris spectral sequence for the covering by open stars (for $i \in I$ ),

$$
V_{\{i\}}=\delta^{-1}\left(U_{\{i\}} \cap \triangleright_{I}\right)
$$

of the vertices of $\triangleright_{I}$. Set $V_{J}=\delta^{-1}\left(U_{J} \cap \triangleright_{I}\right)$. The groups $E_{1}^{a, b}$ are cohomology groups of multi-intersections of open sets in this covering, and were computed in [GHM], Lemma 18.5 ,

$$
\begin{aligned}
E_{1}^{a, b} & =\bigoplus_{\substack{|J|=a+1 \\
J \subset I}} W^{v} H^{b}\left(\bigcap_{j \in J} V_{\{j\}} ; \mathbf{E}\right)=\bigoplus_{\substack{|J|=a+1 \\
J \subset I}} W^{v} H^{b}\left(V_{J} ; \mathbf{E}\right) \\
& =\bigoplus_{\substack{|J|=a+1 \\
J \subset I}} H^{b}\left(\mathfrak{N}_{P}, E\right)_{\geqq v_{P(J)}} .
\end{aligned}
$$

The $E_{1}$ differential is given (up to sign) by inclusion, so the argument of [GHM], $\S 18.7$ applies here as well: the spectral sequence collapses at $E_{2}$, which has only two possibly nonzero columns: $E_{2}^{0, b}=H^{b}\left(\mathfrak{N}_{P}, E\right)_{\geqq v_{P}}$ and, using (12.1.3),

$$
E_{2}^{|I|-1, b}=\frac{H^{b}\left(\mathfrak{N}_{P}, E\right)_{\geqq v_{P(I)}}}{\sum_{\substack{|K|=|I|-1 \\ K \subset I}} H^{b}\left(\mathfrak{N}_{P}, E\right)_{\geqq v_{P(K)}}}=H^{b}\left(\mathfrak{N}_{P}, E\right)_{\left\lceil v_{P}, I\right\rfloor}
$$


which contributes to $W^{v} H^{*}\left(\delta^{-1} S t\left(\triangleright_{I}\right), \mathbf{E}\right)$ in degree $|I|-1+b$. So we obtain a split short exact sequence (with $c=|I|-1+b$ ),

$$
0 \rightarrow H^{c-|I|+1}\left(\mathfrak{N}_{P}, E\right)_{\left\lceil v_{P}, I\right\rfloor} \rightarrow W^{v} H^{c}\left(\mathscr{L}_{y} \cap \bar{X}_{Q} ; \mathbf{E}\right) \rightarrow H^{c}\left(\mathfrak{N}_{P}, E\right)_{\geqq v_{P}} \rightarrow 0
$$

which completes the proof of (12.6.4).

Step 2. As in $[\mathrm{GHM}], \S 18.11$, the long exact sequence for the pair (12.6.2) splits into split short exact sequences,

$$
0 \rightarrow H^{c}\left(B_{\varepsilon} \cap N_{y}\right) \rightarrow H^{c}\left(\partial B_{\varepsilon} \cap N_{y} \cap \bar{X}_{Q}\right) \rightarrow H^{c+1}\left(B_{\varepsilon} \cap N_{y}, \partial B_{\varepsilon} \cap N_{y} \cap \bar{X}_{Q}\right) \rightarrow 0 .
$$

But $H^{*}\left(B_{\varepsilon} \cap N_{y}\right)=H^{*}\left(B_{\varepsilon}\right)=H^{*}\left(\mathfrak{N}_{P}, E\right)_{\geqq v_{P}}$ is the stalk cohomology at $y$ of the weighted cohomology sheaf. This kills the second summand in (12.6.4), leaving

$$
\mathbf{H}_{y}^{m}\left(\mathbf{B}^{\bullet}\right) \cong H^{m}\left(B_{\varepsilon} \cap N_{y}, \partial B_{\varepsilon} \cap N_{y} \cap \bar{X}_{Q} ; \mathbf{W}^{v} \mathbf{C}^{\bullet}(\mathbf{E})\right) \cong H^{m-|I|}\left(\mathfrak{N}_{P}, E\right)_{\left\lceil v_{P}, I\right\rfloor} .
$$

Step 3. We briefly indicate why the isomorphism (12.6.5) extends to an isomorphism of flat vector bundles on $X_{P}$,

$$
\mathbf{H}^{m}\left(\mathbf{B}^{\bullet}\right) \cong H^{m-|I|}\left(\mathfrak{N}_{P}, E\right)_{\left\lceil v_{P}, I\right\rfloor} \times_{\Gamma_{L(P)}} L_{P} / K_{P} A_{P}
$$

(where $\Gamma_{L(P)}=v_{P}(\Gamma \cap P)$ is the projection of $\Gamma \cap P$ to the Levi quotient $L_{P}$ and where it acts on $H^{*}\left(\mathfrak{N}_{P}, E\right)$ by conjugation). Let $i: X \rightarrow \bar{X}$ denote the inclusion. In [GHM], §17, special differential forms are used in order to identify the restriction $\mathbf{H}^{m}\left(R i_{*} \mathbf{E}\right) \mid X_{P}$ with the flat vector bundle

$$
H^{m}\left(\mathfrak{N}_{P}, E\right)_{\left\lceil v_{P}, \phi\right\rfloor} \times \Gamma_{\Gamma_{L(P)}} L_{P} / K_{P} A_{P}
$$

But each of the cohomology groups appearing in Step 2 (above) is an $L_{P}$-submodule of $H^{*}\left(\mathfrak{N}_{P}, E\right)$ and the corresponding bundle on $X_{P}$ is a sub-bundle of $\mathbf{H}^{*} R i_{*}(\mathbf{E}) \mid X_{P}$ (while the shift by $|I|$ corresponds to tensoring with a trivial vector bundle on $X_{P}$ ). So it suffices to verify that the stalk cohomology modules agree at the basepoint, which we have done.

12.7. Kostant's theorem. In this section we will use Kostant's theorem [Ko] to explicitly evaluate the cohomology group (12.5.1). Let $\mathbf{B} \subset \mathbf{G}$ be a Borel subgroup (over $\mathbb{C}$ ), chosen so that $\mathbf{B}(\mathbb{C}) \subset \mathbf{P}_{\mathbf{0}}(\mathbb{C}) \subset \mathbf{P}(\mathbb{C})$. Choose a maximal torus $\mathbf{T}$ (over $\mathbb{C}$ ) of $\mathbf{G}$ so that

$$
\mathbf{S}_{\mathbf{P}}(\mathbb{C}) \subset \mathbf{S}_{\mathbf{0}}(\mathbb{C}) \subset \mathbf{T}(\mathbb{C}) \subset \mathbf{B}_{\mathbf{L}}(\mathbb{C})
$$

where $\mathbf{B}_{\mathbf{L}}=\mathbf{B} \cap \mathbf{L}_{\mathbf{P}}$ is the corresponding Borel subgroup of $\mathbf{L}_{\mathbf{P}}$. This gives rise to root systems $\Phi_{G}=\Phi(\mathbf{G}(\mathbb{C}), \mathbf{T}(\mathbb{C}))$ and $\Phi_{L}=\Phi\left(\mathbf{L}_{\mathbf{P}}(\mathbb{C}), \mathbf{T}(\mathbb{C})\right)$ with positive roots $\Phi_{G}^{+}=\Phi\left(\mathscr{U}_{\mathbf{B}}(\mathbb{C}), \mathbf{T}(\mathbb{C})\right)$ and $\Phi_{L}^{+}=\Phi_{L} \cap \Phi_{G}^{+}$(determined by the Borel subgroups $\mathbf{B} \subset \mathbf{G}$ and $\mathbf{B}_{\mathbf{L}} \subset \mathbf{L}_{\mathbf{P}}$ respectively). Let $\rho_{B}=\frac{1}{2} \sum_{\alpha \in \Phi_{G}^{+}} \alpha$.

Let $W_{G}=W(\mathbf{G}(\mathbb{C}), \mathbf{T}(\mathbb{C}))$ denote the Weyl group of $\mathbf{G}(\mathbb{C})$ and let $W_{P}=W\left(\mathbf{L}_{\mathbf{P}}(\mathbb{C}), \mathbf{T}(\mathbb{C})\right)$ denote the Weyl group of $\mathbf{L}_{\mathbf{P}}(\mathbb{C})$. The choice of $\mathbf{B}$ determines a length function $\ell$ on $W_{G}$. Let $W_{P}^{1} \subset W_{G}$ denote the set of Kostant representatives: the 
unique elements of minimal length from each of the cosets $W_{P} x \in W_{P} \backslash W_{G}$. As in [Sp], §10.2 or [Vo], \$3.2.1, it may also be described as the set

$$
W_{P}^{1}=\left\{w \in W_{G} \mid w^{-1}\left(\Phi_{L}^{+}\right) \subset \Phi_{G}^{+}\right\}
$$

(and depends on the choice of $\mathbf{B}_{\mathbf{L}} \subset \mathbf{L}_{\mathbf{P}}$ ).

If $\beta \in \chi^{*}(\mathbf{T}(\mathbb{C}))$ is $\mathbf{B}_{\mathbf{L}}$-dominant, let us write $V_{\beta}^{L}$ for the irreducible $\mathbf{L}_{\mathbf{P}}$-representation with highest weight $\beta$. Let $\lambda_{B} \in \chi^{*}(\mathbf{T}(\mathbb{C}))$ be the highest weight of the irreducible representation $E$ of $\mathbf{G}$. Kostant's theorem states that for all $w \in W_{P}^{1}$, the weight $w\left(\lambda_{B}+\rho_{B}\right)-\rho_{B}$ is $\mathbf{B}_{\mathbf{L}}$-dominant, and that as an $\mathbf{L}_{\mathbf{P}}$-module, the cohomology group $H^{i}\left(\mathfrak{N}_{P}, E\right)$ is isomorphic to

$$
\bigoplus\left\{V_{w\left(\lambda_{B}+\rho_{B}\right)-\rho_{B}}^{L} \mid w \in W_{P}^{1} \text { and } \ell(w)=i\right\}
$$

If $w \in W_{G}$ then the character $w\left(\lambda_{B}+\rho_{B}\right)-\rho_{B}-v$ of $\mathbf{S}_{\mathbf{0}}$ is trivial on $\mathbf{S}_{\mathbf{G}}$ so we may define

$$
I_{v}(w)=\left\{\alpha \in \Delta_{P} \mid\left\langle\left(w\left(\lambda_{B}+\rho_{B}\right)-\rho_{B}-v\right) \mid \mathbf{S}_{\mathbf{P}}^{\prime}, t_{\alpha}\right\rangle<0\right\}
$$

where $\left\{t_{\alpha}\right\}$ form the basis of the cocharacter group $\chi_{*}^{\mathbb{Q}}\left(\mathbf{S}_{\mathbf{P}}^{\prime}\right)$ which is dual to the basis $\Delta_{P}$ of simple roots, cf. (12.1.1). So in the notation of (12.1.1),

$$
I_{v}(w)=I_{v}(\gamma) \quad \text { where } \gamma=\left(w\left(\lambda_{B}+\rho_{B}\right)-\rho_{B}\right) \mid \mathbf{S}_{\mathbf{P}}
$$

To summarize we have:

12.8. Proposition. Let $\mathbf{P}$ be a standard rational parabolic subgroup of $\mathbf{G}$. Let $v_{P}=v \mid \mathbf{S}_{\mathbf{P}} \in \chi_{\mathbb{Q}}^{*}\left(\mathbf{S}_{\mathbf{P}}\right)$ be the character which is determined by the weight profile $v \in \chi_{\mathbb{Q}}^{*}\left(\mathbf{S}_{\mathbf{0}}\right)$. Let $\lambda_{B}$ denote the highest weight of the irreducible representation $E$ of $\mathbf{G}$. Let $I \subset \Delta_{P}$ be a subset corresponding to a choice of standard rational parabolic subgroup $\mathbf{Q} \supset \mathbf{P}$. Then Kostant's theorem determines an isomorphism of graded $\mathbf{L}_{\mathbf{P}}$-modules,

$$
H^{*}\left(\mathfrak{N}_{P}, E\right)_{\left\lceil v_{P}, I\right\rfloor} \cong \bigoplus_{\substack{w \in W_{P}^{1} \\ I_{v}(w)=I}} V_{w\left(\lambda_{B}+\rho_{B}\right)-\rho_{B}}^{L}[-\ell(w)]
$$

where the sum is taken over all $w \in W_{P}^{1}$ such that $I_{v}(w)=I$, and where $V_{\beta}^{L}[-m]$ means that the irreducible $\mathbf{L}_{\mathbf{P}}$-module $V_{\beta}^{L}$ appears in degree $m$.

\section{Lefschetz numbers}

13.1. In this section we recall the Lefschetz fixed point theorem for hyperbolic correspondences from $[\mathrm{GM} 2], \S 10.3$.

Suppose $\bar{C}, \bar{X}$ and $\bar{Y}$ are compact subanalytic spaces and that $c=\left(c_{1}, c_{2}\right): \bar{C} \rightarrow \bar{X} \times \bar{Y}$ is a subanalytic mapping. (The bars are used so that the notation here will agree with that in the rest of the paper.) Let $\mathbf{S}^{\bullet} \in D^{b}(\bar{X})$ be a (bounded from below) complex of (cohomologically) constructible sheaves on $\bar{X}$ and let $\mathbf{T}^{\bullet} \in D^{b}(\bar{Y})$ be a (bounded from below) complex of (cohomologically) constructible sheaves on $\bar{Y}$. Since 
$c$ is proper we have $c_{*}=c_{\text {! }}$. A lift of the correspondence $\bar{C}$ to the sheaf level ([Ve], [GI], [Bo5]) is a morphism

$$
\Phi: c_{2}^{*} \mathbf{T}^{\bullet} \rightarrow c_{1}^{!} \mathbf{S}^{\bullet}
$$

Such a morphism induces a homomorphism $H^{*}\left(\bar{Y} ; \mathbf{T}^{\bullet}\right) \rightarrow H^{*}\left(\bar{X} ; \mathbf{S}^{\bullet}\right)$ as follows. First apply $\left(c_{1}\right)$ ! and adjunction to obtain a morphism

$$
\left(c_{1}\right)_{!} c_{2}^{*} \mathbf{T}^{\bullet} \rightarrow\left(c_{1}\right)_{!} c_{1}^{!} \mathbf{S}^{\bullet} \rightarrow \mathbf{S}^{\bullet}
$$

Let $p: \bar{X} \rightarrow$ pt and $q: \bar{Y} \rightarrow$ pt be the map to a point. Then the diagram

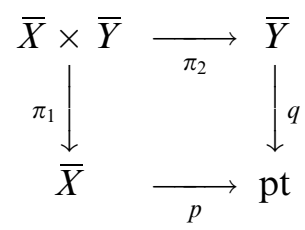

is a fiber square so there is an adjunction natural transformation [GM2], (2.6b), $q_{!}\left(\pi_{2}\right)_{*} \rightarrow p_{*}\left(\pi_{1}\right)_{!}$. Apply $q_{\text {! }}$ to the adjunction morphism $\mathbf{T}^{\bullet} \rightarrow\left(c_{2}\right)_{*} c_{2}^{*} \mathbf{T}^{\bullet}$ and use (13.1.2) to obtain

$$
\begin{aligned}
q_{!} \mathbf{T}^{\bullet} & \rightarrow q_{!}\left(c_{2}\right)_{*} c_{2}^{*} \mathbf{T}^{\bullet}=q_{!}\left(\pi_{2}\right)_{*} c_{*} c_{2}^{*} \mathbf{T}^{\bullet} \\
& \rightarrow p_{*}\left(\pi_{1}\right)_{!} c_{*} c_{2}^{*} \mathbf{T}^{\bullet}=p_{*}\left(c_{1}\right)_{!} c_{2}^{*} \mathbf{T}^{\bullet} \rightarrow p_{*} \mathbf{S}^{\bullet}
\end{aligned}
$$

This morphism induces the desired mapping on cohomology. (It may also be constructed by applying $p_{!}\left(c_{2}\right)_{*}$ to $(13.1 .1)$ rather than $q_{*}\left(c_{1}\right)_{!}$.)

In what follows, we suppose $\bar{X}=\bar{Y}$ and $\mathbf{S}^{\bullet}=\mathbf{T}^{\bullet}$, so $c=\left(c_{1}, c_{2}\right): \bar{C} \rightarrow \bar{X} \times \bar{X}$ is a correspondence on $\bar{X}$ and $\Phi: c_{2}^{*} \mathbf{S}^{\bullet} \rightarrow c_{1}^{!} \mathbf{S}^{\bullet}$ is a lift to the sheaf level. The Lefschetz fixed point theorem states that the resulting Lefschetz number

$$
L\left(\mathbf{S}^{\bullet}, \bar{C}\right)=\sum_{i \geqq 0} \operatorname{Tr}\left(\Phi^{*}: H^{i}\left(\bar{X} ; \mathbf{S}^{\bullet}\right) \rightarrow H^{i}\left(\bar{X} ; \mathbf{S}^{\bullet}\right)\right)=\sum_{F} L\left(\mathbf{S}^{\bullet}, \bar{C}, F\right)
$$

is a sum of locally defined contributions $L\left(\mathbf{S}^{\bullet}, \bar{C}, F\right)$, one for each connected component $F \subset \bar{C}$ of the fixed point set of the correspondence $\bar{C}$.

Let $F \subset \bar{C}$ be a connected component of the fixed point set and suppose that the correspondence $\bar{C}$ is weakly hyperbolic $(\$ 11.7)$ near $F^{\prime}=c_{1}(F)=c_{2}(F)$ with indicator mapping $t: W \rightarrow \mathbb{R}_{\geqq 0} \times \mathbb{R}_{\geqq 0}$. (This means that $W \subset \bar{X}$ is a neighborhood of $F^{\prime}$, that $t$ is a proper subanalytic mapping such that $t^{-1}(0,0)=F^{\prime}$, and that for all $x \in c_{1}^{-1}(W) \cap c_{2}^{-1}(W)$ we have $t_{1} c_{1}(x) \leqq t_{1} c_{2}(x)$ and $t_{2} c_{1}(x) \geqq t_{2} c_{2}(x)$.) Denote by $h$ and $j$ the inclusions

$$
F^{\prime} \stackrel{h}{\rightarrow} t^{-1}\left(\mathbb{R}_{\geqq 0} \times\{0\}\right) \stackrel{j}{\rightarrow} \bar{X}
$$

of $F^{\prime}$ into the "expanding set" or "unstable manifold" $F^{-}=t^{-1}\left(\mathbb{R}_{\geqq 0} \times\{0\}\right)$, and of $F^{-}$ into $\bar{X}$. 
Let $\mathbf{A}^{\bullet}=h^{!} j^{*}\left(\mathbf{S}^{\bullet}\right)$ as in $\$ 12.4$. Then the lift $\Phi$ determines a lift $\Psi^{\prime}: c_{2}^{*} \mathbf{A}^{\bullet} \rightarrow c_{1}^{!} \mathbf{A}^{\bullet}$ which, by adjunction, induces an endormorphism $\Psi: \mathbf{A}^{\bullet} \rightarrow \mathbf{A}^{\bullet}$ (which covers the identity mapping on $F^{\prime}$ ). In [GM2] we prove:

13.2. Theorem. The contribution $L\left(\mathbf{S}^{\bullet}, \bar{C}, F\right)$ of $F$ to the global Lefschetz number $L\left(\mathbf{S}^{\bullet}, \bar{C}\right)$ is given by

$$
L\left(\mathbf{S}^{\bullet}, \bar{C}, F\right)=\sum_{i \geqq 0}(-1)^{i} \operatorname{Tr}\left(\Psi^{*}: H^{i}\left(F^{\prime} ; \mathbf{A}^{\bullet}\right) \rightarrow H^{i}\left(F^{\prime} ; \mathbf{A}^{\bullet}\right)\right) .
$$

Moreover, if $F^{\prime}=\coprod_{\alpha=1}^{m} F_{\alpha}^{\prime}$ is stratified so that the pointwise Lefschetz number $n(x)=\sum_{i \geqq 0}(-1)^{i} \operatorname{Tr}\left(\Psi_{x}^{*}: H_{x}^{i}\left(\mathbf{A}^{\bullet}\right) \rightarrow H_{x}^{i}\left(\mathbf{A}^{\bullet}\right)\right)$ is constant on each stratum, then the local contribution is the sum over strata,

$$
L\left(\mathbf{S}^{\bullet}, \bar{C}, F\right)=\sum_{\alpha=1}^{m} \chi_{c}\left(F_{\alpha}^{\prime}\right) n\left(x_{\alpha}\right)
$$

where $x_{\alpha} \in F_{\alpha}^{\prime}$ and where $\chi_{c}$ denotes the Euler characteristic with compact supports. (See [KS], Prop. 9.6.12 for a related result.) The right hand side of (13.2.1) is the Euler characteristic $\chi\left(F^{\prime} ; n\right)$ of the constructible function $n(x)$, as discussed in [Mac].

13.3. Morphisms and weighted cohomology. In this section we show how to lift any morphism to the weighted cohomology sheaf. As in $\$ 2$, G denotes a connected linear reductive algebraic group defined over $\mathbb{Q}, D$ denotes the associated symmetric space, $K^{\prime}=A_{G} K\left(x_{0}\right)$ is the stabilizer in $G$ of a fixed basepoint $x_{0} \in D, \Gamma \subset \mathbf{G}(\mathbb{Q})$ is a neat arithmetic group and $X=\Gamma \backslash D$. As in $\S 12.2$ let $\tau: G \rightarrow G L(E)$ be a finite dimensional irreducible representation on some complex vector space. It gives rise to the local coefficient system (flat homogeneous vector bundle) $\mathbf{E}=\left(G / K^{\prime}\right) \times_{\Gamma} E$ which is the quotient of $\left(G / K^{\prime}\right) \times E$ under the equivalence relation $\left(x K^{\prime}, v\right) \sim\left(\gamma x K^{\prime}, \tau(\gamma) v\right)$ for all $\gamma \in \Gamma$. Denote by $\left[x K^{\prime}, v\right] \in \mathbf{E}$ the resulting equivalence class. Let $\mathbf{P}_{\mathbf{0}}$ be the standard minimal rational parabolic subgroup with $\mathbf{S}_{\mathbf{0}}=\mathbf{S}_{\mathbf{P}_{\mathbf{0}}}$. Fix $v \in \chi_{\mathbb{Q}}^{*}\left(\mathbf{S}_{\mathbf{0}}\right)$ so that $v \mid \mathbf{S}_{\mathbf{G}}$ coincides with the character by which $\mathbf{S}_{\mathbf{G}}$ acts on $E$ and let $\mathbf{W}^{v} \mathbf{C}^{\bullet}(\bar{X} ; \mathbf{E})$ denote the resulting weighted cohomology complex of sheaves on $\bar{X}$.

Suppose $\Gamma^{\prime} \subset \Gamma$ is a subgroup of finite index, set $C=\Gamma^{\prime} \backslash G / K$, and let $f: C \rightarrow X$ be a morphism, i.e., there exists $h \in \mathbf{G}(\mathbb{Q})$ such that $h \Gamma^{\prime} h^{-1} \subset \Gamma$ and $f\left(\Gamma^{\prime} x K\right)=\Gamma h x K$. Let $\mathbf{E}^{\prime} \rightarrow C$ be the local coefficient system on $C$ which is determined by the representation $\tau: G \rightarrow G L(E)$. The morphism $f$ is covered by a mapping $\mathbf{E}^{\prime} \rightarrow \mathbf{E}$ of local systems given by $[x K, v] \mapsto[h x K, \tau(h) v]$. This mapping is easily seen to be well defined, and it induces an isomorphism of local systems $\mathbf{E}^{\prime} \cong f^{*}(\mathbf{E})$ on $C$. Since $f: C \rightarrow X$ is an unramified finite covering, it further induces a canonical quasi-isomorphism of the sheaves of smooth differential forms with coefficients in this local system, $f^{*} \mathbf{\Omega}^{\bullet}(X ; \mathbf{E}) \rightarrow \boldsymbol{\Omega}^{\bullet}\left(C ; \mathbf{E}^{\prime}\right)$.

The morphism $f: C \rightarrow X$ admits a unique continuous extension $\bar{f}: \bar{C} \rightarrow \bar{X}$ to the reductive Borel-Serre compactifications (Lemma 6.3). If $i_{C}: C \rightarrow \bar{C}$ and $i_{X}: X \rightarrow \bar{X}$ denote the inclusions then the adjunction mapping [GM2], equation (2.5a), 


$$
\bar{f}^{*}\left(i_{X}\right)_{*} \mathbf{\Omega}^{\bullet}(X ; \mathbf{E}) \stackrel{\cong}{\rightrightarrows}\left(i_{C}\right)_{*} f^{*} \mathbf{\Omega}^{\bullet}(X ; \mathbf{E}) \stackrel{\cong}{\rightrightarrows}\left(i_{C}\right)_{*} \mathbf{\Omega}^{\bullet}(C ; \mathbf{E})
$$

is a quasi-isomorphism. It is easy to see that this induces a quasi-isomorphism

$$
\bar{f}^{*} \mathbf{W}^{v} \mathbf{C}^{\bullet}(\bar{X} ; \mathbf{E}) \rightarrow \mathbf{W}^{v} \mathbf{C}^{\bullet}\left(\bar{C} ; \mathbf{E}^{\prime}\right)
$$

of weighted cohomology sheaves. (In fact the whole construction of the weighted cohomology sheaf on $\bar{X}$ pulls back to the construction of weighted cohomology on $\bar{C}$.)

13.4. Hecke correspondences and weighted cohomology. Let $g \in \mathbf{G}(\mathbb{Q})$. Then $g$ gives rise to a Hecke correspondence $\left(c_{1}, c_{2}\right): \bar{C} \rightarrow \bar{X}$. Here, $\bar{C}$ is the reductive Borel-Serre compactification of $C=\Gamma^{\prime} \backslash G / K$ with $\Gamma^{\prime}=\Gamma \cap g^{-1} \Gamma g$. Both mappings $c_{1}$ and $c_{2}$ are finite so there are natural isomorphisms of functors $c_{i}^{*} \cong c_{i}^{!}$and $\left(c_{i}\right)_{*} \cong\left(c_{i}\right)_{!}$(for $\left.i=1,2\right)$. From the preceding paragraph we obtain a canonical lift

$$
\Phi: c_{2}^{*} \mathbf{W}^{v} \mathbf{C}^{\bullet}(\bar{X} ; \mathbf{E}) \rightarrow c_{1}^{!} \mathbf{W}^{v} \mathbf{C}^{\bullet}(\bar{X} ; \mathbf{E})
$$

to the weighted cohomology sheaves, which is given by the composition

$$
c_{2}^{*} \mathbf{W}^{v} \mathbf{C}^{\bullet}(\bar{X} ; \mathbf{E}) \stackrel{\cong}{\rightrightarrows} \mathbf{W}^{v} \mathbf{C}^{\bullet}(\bar{C} ; \mathbf{E}) \stackrel{\cong}{\leftrightarrows} c_{1}^{*} \mathbf{W}^{v} \mathbf{C}^{\bullet}(\bar{X} ; \mathbf{E}) \cong c_{1}^{!} \mathbf{W}^{v} \mathbf{C}^{\bullet}(\bar{X} ; \mathbf{E}) .
$$

13.5. Computation of the local contribution. For the remainder of $\S 13$, fix a Hecke correspondence $\bar{C} \rightrightarrows \bar{X}$ which is determined by some element $g \in \mathbf{G}(\mathbb{Q})$. Fix a regular $\Gamma$ equivariant parameter $b \in \mathscr{B}$ which is so large that the resulting tilings $\left\{D^{P}\right\}$ of $\bar{D},\left\{X^{P}\right\}$ of $\bar{X}$ and $\left\{C^{P}\right\}$ of $\bar{C}$ are narrow (\$6.11) with respect to the Hecke correspondence. Choose $\mathbf{t} \in A_{P_{0}}(>1)$ to be regular, dominant, and sufficiently close to $\mathbf{1}$ as in Proposition 11.2, with resulting shrink homeomorphism $\operatorname{Sh}(\mathbf{t})$, and let $\left(c_{1}^{\prime}, c_{2}^{\prime}\right): \bar{C} \rightrightarrows \bar{X}$ be the resulting modified correspondence. It is easy to see that $\operatorname{Sh}(\mathbf{t})^{*}\left(\mathbf{W}^{v} \mathbf{C}^{\bullet}\right) \cong \mathbf{W}^{v} \mathbf{C}^{\bullet}$ so we may consider (13.4.1) to be a lift of the modified correspondence as well.

Suppose the Hecke correspondence preserves some stratum $C_{P}$. According to Proposition 7.3, locally near $C_{P}$ the correspondence is isomorphic to the parabolic Hecke correspondence $\Gamma_{P}^{\prime} \backslash D[P] \rightrightarrows \Gamma_{P} \backslash D[P]$ which is given by some $y \in \mathbf{P}(\mathbb{Q}) \cap \Gamma g \Gamma$ and to which we may associate a decomposition $\Delta_{P}=\Delta_{P}^{+} \cup \Delta_{P}^{-} \cup \Delta_{P}^{0}$ of the simple roots. Suppose that $C_{P}$ contains fixed points and denote by $F_{P}(e) \subset C_{P}$ the set of fixed points with characteristic element $e \in \Gamma_{L} \bar{y} \Gamma_{L} \subset \mathbf{L}_{\mathbf{P}}(\mathbb{Q})$.

By Proposition 8.4 the torus factor $a_{e} \in A_{P}$ of $e$ coincides with the torus factor $a_{y}$ so the set $\Delta_{P}^{+}$(resp. $\Delta_{P}^{-}$, resp. $\left.\Delta_{P}^{0}\right)$ consists of those simple roots $\alpha \in \Delta_{P}$ for which $\alpha\left(a_{e}\right)<0$ $($ resp. $>0$, resp. $=0)$. Hence we may write $\Delta_{P}^{+}=\Delta_{P}^{+}(e)\left(\right.$ resp. $\Delta_{P}^{-}=\Delta_{P}^{-}(e)$, resp. $\left.\Delta_{P}^{0}=\Delta_{P}^{0}(e)\right)$.

As in $\S 12.7$, choose a Borel pair $\mathbf{T}(\mathbb{C}) \subset \mathbf{B}(\mathbb{C})$ so that (12.7.1) holds. Assume the local system $\mathbf{E}$ arises from an irreducible representation of $\mathbf{G}$ with highest weight $\lambda_{B} \in \chi^{*}(\mathbf{T}(\mathbb{C}))$. Let $\rho_{B}=\frac{1}{2} \sum_{\alpha \in \Phi_{G}^{+}} \alpha \in \chi^{*}(\mathbf{T}(\mathbb{C}))$ denote the half-sum of the positive roots. Let
$r=\left[\Gamma \cap \mathscr{U}_{P}: \Gamma^{\prime} \cap \mathscr{U}_{P}\right]$.

13.6. Theorem. The contribution to the Lefschetz number from the fixed point constituent $F_{P}(e)$ is: 


$$
r \chi_{c}\left(F_{P}(e)\right)(-1)^{\left|\Delta_{P}^{+}\right|} \sum_{\substack{w \in W_{P}^{1} \\ I_{v}(w)=\Delta_{P}^{+}(e)}}(-1)^{\ell(w)} \operatorname{Tr}\left(e^{-1} ; V_{w\left(\lambda_{B}+\rho_{B}\right)-\rho_{B}}^{L}\right)
$$

where $I_{v}(w)$ is defined in (12.7.2).

In $[\mathrm{GKM}], \S 7.14$ the Lefschetz formula in the adelic setting is described but not proven. The missing ingredient is the proof of the formula for the local contribution $L_{Q}(\gamma)$ which appears on page 534. (This formula differs slightly from (13.6.1) because the factor $r \chi_{c}\left(F_{P}(e)\right)$ is absorbed by the orbital integral in $\left.[\mathrm{GKM}].\right)$ Theorem 13.6 thus provides the proof of this formula, so it completes the proof of Theorem B (7.14) of [GKM]. The proof of Theorem 13.6 will occupy the rest of this section.

13.7. The nilmanifold correspondence. The Hecke correspondence $C \rightrightarrows X$ extends to a correspondence on the Borel-Serre compactification

$$
\tilde{C} \rightrightarrows \tilde{X}
$$

which is compatible with the projection $\mu: \tilde{X} \rightarrow \bar{X}$ to the reductive Borel-Serre compactification. Let $w^{\prime} \in F_{P}(e)$ and set $w=c_{1}\left(w^{\prime}\right)=c_{2}\left(w^{\prime}\right)$. The restriction of the correspondence to the relevant Borel-Serre stratum is given by

$$
\begin{aligned}
Y_{P}^{\prime}=\Gamma_{P}^{\prime} \backslash P / K_{P} A_{P} & \rightrightarrows Y_{P}=\Gamma_{P} \backslash P / K_{P} A_{P}, \\
\Gamma_{P}^{\prime} x K_{P} A_{P} & \mapsto\left(\Gamma_{P} x K_{P} A_{P}, \Gamma_{P} y x K_{P} A_{P}\right)
\end{aligned}
$$

(Here, $\Gamma_{P}^{\prime}=\Gamma_{P} \cap y^{-1} \Gamma_{P} y$.) The fibers $N_{P}=\mu^{-1}(w) \subset Y_{P}$ and $N_{P}^{\prime}=\left(\mu^{\prime}\right)^{-1}\left(w^{\prime}\right) \subset Y_{P}^{\prime}$ are nilmanifolds isomorphic to $\Gamma_{\mathscr{U}} \backslash \mathscr{U}_{P}$ and $\Gamma_{\mathscr{U}}^{\prime} \backslash \mathscr{U}$ respectively, where $\Gamma_{\mathscr{U}}=\Gamma_{P} \cap \mathscr{U}_{P}$ and $\Gamma_{\mathscr{U}}^{\prime}=\Gamma_{P}^{\prime} \cap \mathscr{U}_{P}=\Gamma \cap y^{-1} \Gamma_{\mathscr{U}} y$. So the correspondence 13.7.1 restricts to a correspondence $N_{P}^{\prime} \rightrightarrows N_{P}$ which will be described below. The following diagram may help in sorting out these spaces.

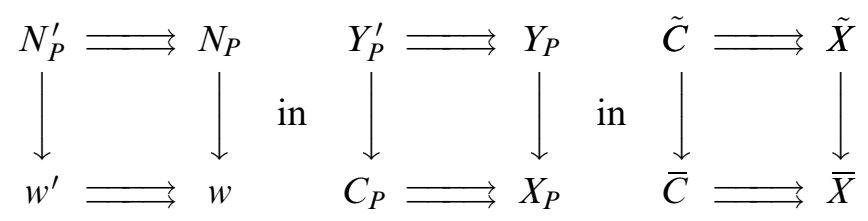

13.8. Lemma. Let $\phi: L_{P} \rightarrow G L\left(H^{*}\left(\mathfrak{N}_{P}, E\right)\right)$ denote the adjoint representation of the Levi quotient $L_{P}$ on the Lie algebra cohomology of $\mathfrak{N}_{P}$. Let $w^{\prime} \in F_{P}(e)$ be a fixed point in $C_{P}$ with characteristic element $e \in L_{P}$. Then the nilmanifold correspondence $\left(c_{1}, c_{2}\right): N_{P}^{\prime} \rightrightarrows N_{P}$ induces a mapping $\left(c_{1}\right)_{*} c_{2}^{*}: H^{*}\left(N_{P}, \mathbf{E}\right) \rightarrow H^{*}\left(N_{P}, \mathbf{E}\right)$ on cohomology which, under the Nomizu-van Est isomorphism $H^{*}\left(N_{P}, \mathbf{E}\right) \cong H^{*}\left(\mathfrak{N}_{P}, E\right)$ may be identified with the homomorphism

$$
r \phi\left(e^{-1}\right)
$$

where $r=\left[\Gamma_{\mathscr{U}}: \Gamma_{\mathscr{U}}^{\prime}\right]$.

13.9. Proof. First we find equations for the nilmanifold correspondence. Choose a 
lift $x K_{P} A_{P} \in D=P / K_{P} A_{P}$ of the fixed point $w^{\prime}=\Gamma_{P}^{\prime} x K_{P} A_{P} \mathscr{U}_{P} \in C_{P}$. This determines a parametrization of the nilmanifold $N_{P}$ by

$$
\begin{aligned}
\Gamma_{\mathscr{U}} \backslash \mathscr{U} & \rightarrow N_{P} \subset Y_{P}=\Gamma_{P} \backslash P / K_{P} A_{P}, \\
\Gamma_{\mathscr{U}} z & \mapsto \Gamma_{P} z x K_{P} A_{P},
\end{aligned}
$$

and similarly $\Gamma_{\mathscr{U}}^{\prime} \backslash \mathscr{U} \rightarrow N_{P}^{\prime}$ by $\Gamma_{\mathscr{U}}^{\prime} z \mapsto \Gamma_{P}^{\prime} z x K_{P} A_{P}$.

Since $w^{\prime}$ is fixed, we have $\Gamma_{P} x \mathscr{U}_{P} K_{P} A_{P}=\Gamma_{P} y x \mathscr{U}_{P} K_{P} A_{P}$ hence there exists $\gamma \in \Gamma_{P}$ and $u \in \mathscr{U}_{P}$ so that $\gamma y u x K_{P} A_{P}=x K_{P} A_{P}$, in other words, so that $\gamma y u$ fixes the point $x K_{P} A_{P}$ in the Borel-Serre boundary component $P / K_{P} A_{P}$. Then $e=v_{P}(\gamma y)=v_{P}(\gamma y u)$ is the characteristic element of the fixed point $w^{\prime}$. Define $\Gamma_{\mathscr{U}}^{\prime} \backslash \mathscr{U} \rightrightarrows \Gamma_{\mathscr{U}} \backslash \mathscr{U}$ by

$$
\Gamma_{\mathscr{U}^{\prime}}^{\prime} z \mapsto\left(\Gamma_{\mathscr{U}} z, \Gamma_{\mathscr{U}}(\gamma y) z u^{-1}(\gamma y)^{-1}\right) .
$$

A simple calculation shows that the following diagram commutes:

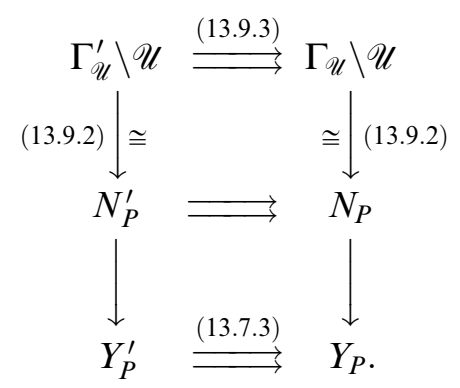

Next we will apply the theorem of Nomizu [Nol] and van Est [E] to this correspondence. The local system $\mathbf{E} \rightarrow X$ which is defined by the representation $\tau: G \rightarrow G L(E)$ extends canonically to a local system on the Borel-Serre compactification $\tilde{X}$. Its restriction to the nilmanifold $N_{P}$ is given by the quotient $\mathbf{E} \mid N_{P}=\mathscr{U} \times_{\Gamma_{2 /}} E$ under the relation $(z, v) \sim(\gamma z, \tau(\gamma) v)$ (for $\gamma \in \Gamma_{\mathscr{U}}, z \in \mathscr{U}_{P}$, and $\left.v \in E\right)$. The complex $\Omega^{\bullet}\left(N_{P}, \mathbf{E}\right)$ of smooth $\mathbf{E}$ valued differential forms on $N_{P}$ consists of sections of the (flat) vector bundle

$$
\mathbf{C}^{\bullet}\left(N_{P}, \mathbf{E}\right)=\mathscr{U}_{P} \times_{\Gamma_{P}} C^{\bullet}\left(\mathfrak{N}_{P}, E\right)
$$

where $C^{\bullet}\left(\mathfrak{N}_{P}, E\right)=\operatorname{Hom}_{\mathbb{C}}\left(\wedge^{\bullet} \mathfrak{N}_{P}, E\right)$ is the complex of Lie algebra cochains. Let $\phi$ be the representation of $P$ on this complex: if $\wedge^{\bullet} \operatorname{Ad}(p): \wedge^{\bullet} \mathfrak{N}_{P} \rightarrow \wedge^{\bullet} \mathfrak{N}_{P}$ denotes the adjoint action of $p \in P$ on the exterior algebra of $\mathfrak{M}_{P}$, then

$$
\phi(p)(s)=\tau(p) \circ s \circ \wedge^{\bullet} \operatorname{Ad}(p) .
$$

Denote by

$$
\Omega_{\text {inv }}^{\bullet}\left(\mathscr{U}_{P}, E\right)=\left\{\omega: \mathscr{U}_{P} \rightarrow C^{\bullet}\left(\mathfrak{N}_{P}, E\right) \mid \omega(u x)=\phi(u) \omega(x) \text { for all } u, x \in \mathscr{U}_{P}\right\}
$$

the complex of (left) $\mathscr{U}_{P}$-invariant $E$-valued differential forms on $\mathscr{U}_{P}$. Such a differential form is determined by its value $s=\omega(1) \in C^{\bullet}\left(\mathfrak{N}_{P}, E\right)$, and it passes to a differential form 
on $N_{P}$. Denote by $\Omega_{\text {inv }}^{\bullet}\left(N_{P}, \mathbf{E}\right)$ the collection of all such "left"-invariant differential forms. The Nomizu-van Est theorem ([Nol], [E]) states that the inclusion $\Omega_{\text {inv }}^{\bullet}\left(N_{P}, \mathbf{E}\right) \hookrightarrow \Omega^{\bullet}\left(N_{P}, \mathbf{E}\right)$ induces an isomorphism on cohomology. In summary we have a diagram

$$
C^{\bullet}\left(\mathfrak{N}_{P}, E\right) \stackrel{\cong}{\longleftarrow} \Omega_{\text {inv }}^{\bullet}\left(\mathscr{U}_{P}, E\right) \stackrel{\cong}{\longleftarrow} \Omega_{\text {inv }}^{\bullet}\left(N_{P}, \mathbf{E}\right) \hookrightarrow \Omega^{\bullet}\left(N_{P}, \mathbf{E}\right)
$$

of isomorphisms and quasi-isomorphisms. Although the group $P$ does not act on the vector bundle $\mathbf{C}^{\bullet}\left(N_{P}, \mathbf{E}\right)$, it does act on the complex $\Omega_{\text {inv }}^{\bullet}\left(N_{P}, \mathbf{E}\right) \cong \Omega_{\text {inv }}^{\bullet}\left(\mathscr{U}_{P}, E\right)$ of invariant sections by

$$
(p \cdot \omega)(x)=\phi(p)^{-1} \omega\left(p x p^{-1}\right)
$$

and the group $\mathscr{U}_{P}$ acts on this complex by

$$
(u \cdot \omega)(x)=\omega\left(x u^{-1}\right) .
$$

If $\omega \in \Omega_{\text {inv }}^{\bullet}\left(N_{P}, \mathbf{E}\right)$ is given by (13.9.4) then by (13.9.3) its pullback by $c_{2}$ is given by

$$
c_{2}^{*}(\omega)(z)=\phi(\gamma y)^{-1} \omega\left((\gamma y) z u^{-1}(\gamma y)^{-1}\right) .
$$

Evaluating at $z=1$ and using the fact that $\omega$ is left invariant,

$$
c_{2}^{*}(\omega)(1)=\phi(u)^{-1} \phi(\gamma y)^{-1} \omega(1) .
$$

Let $s=\omega(1) \in C^{\bullet}\left(\mathfrak{N}_{P}, E\right)$, suppose $d s=0$ and let $[s] \in H^{*}\left(\mathfrak{N}_{P}, E\right)$ be the resulting cohomology class. Since $\mathscr{U}_{P}$ acts trivially on this cohomology,

$$
c_{2}^{*}([s])=\phi(e)^{-1}[s]
$$

where $e=v_{P}(\gamma y)$ is the characteristic element of the fixed point $w$. Finally, observe that the pushforward mapping $\left(c_{1}\right)_{*}: H^{*}\left(N_{P}^{\prime}, \mathbf{E}\right) \rightarrow H^{*}\left(N_{P}, \mathbf{E}\right)$ is given by multiplication by $r=\left[\Gamma_{\mathscr{U}}: \Gamma_{\mathscr{U}}^{\prime}\right]$. This completes the proof of Lemma 13.8.

13.10. Proof of Theorem 13.6. We will apply the Lefschetz fixed point formula to the modified Hecke correspondence. By Proposition 11.2, after modifying the correspondence by composing with $S h(\mathbf{t})$, the fixed point constituent $F_{P}(e)$ becomes "truncated", that is, it becomes replaced by the intersection $F_{P}^{0}(e)=F_{P}(e) \cap C_{P}^{0}$ of $F_{P}(e)$ with the central tile in $C_{P}$. Denote by $\partial F^{0}=F_{P}(e) \cap \partial C_{P}^{0}$ its intersection with the boundary of the central tile. Set $F^{\prime}=c_{1}\left(F_{P}(e)\right)=c_{2}\left(F_{P}(e)\right)$. Set $E=F^{\prime} \cap X_{P}^{0}=c_{i}\left(F_{P}^{0}(e)\right)$ and $\partial E=F^{\prime} \cap \partial X_{P}^{0}=c_{i}\left(\partial F^{0}\right)$. (Having used up all the letters some time ago, we temporarily re-use the notation $E$ here, hoping the reader will not confuse it with the local system.) Note that $E-\partial E$ is diffeomorphic to $F^{\prime}$.

By Theorem 11.9 the (modified) Hecke correspondence is weakly hyperbolic near $F_{P}(e)$ and an indicator mapping (defined in a neighborhood $U \subset \bar{X}$ of $F^{\prime}$ ) is given by

$$
t(x)=\left(\sum_{\alpha \in \Delta_{P}^{+}} r_{\alpha}^{P}(x)+d_{E} \pi_{P}(x), \sum_{\alpha \in \Delta_{P}^{-}} r_{\alpha}^{P}(x)+\sum_{\alpha \in \Delta_{P}^{0}} r_{\alpha}^{P}(x)\right) .
$$


Let $\mathbf{Q} \supset \mathbf{P}$ be the rational parabolic subgroup corresponding to the subset $I=\Delta_{P}^{+} \subset \Delta_{P}$ consisting of the simple roots for which the Hecke correspondence is (strictly) expanding. Then, in the notation of (2.4.1), $\Delta_{P}=i\left(\Delta_{Q}\right) \amalg \Delta_{P}^{+}$. The partial distance function $r_{\alpha}^{P}$ vanishes on the stratum $X_{Q}$ whenever $\alpha \in \Delta_{P}^{-} \cup \Delta_{P}^{0}$, cf. (3.5.2). Hence $\bar{X}_{Q} \cap U=t^{-1}\left(\mathbb{R}_{\geqq 0} \times\{0\}\right)$ is the "expanding set" of the correspondence.

According to Theorem 13.2 we need to compute the stalk cohomology (at points $w \in E$ ) of the sheaf

$$
\mathbf{A}^{\bullet}=h^{!} j^{*} \mathbf{W}^{v} \mathbf{C}^{\bullet}(\mathbf{E})
$$

where

$$
E \underset{h}{\rightarrow} \bar{X}_{Q} \underset{j}{\rightarrow} \bar{X}
$$

This is best accomplished by decomposing $h$,

$$
E \underset{h_{1}}{\rightarrow} F^{\prime} \underset{h_{2}}{\rightarrow} X_{P} \underset{h_{3}}{\rightarrow} \bar{X}_{Q} \underset{j}{\rightarrow} \bar{X}
$$

Then $\mathbf{B}^{\bullet}=h_{3}^{!} j^{*} \mathbf{W}^{v} \mathbf{C}^{\bullet}(\mathbf{E})$ is the sheaf studied in Theorem 12.5, where we have taken $I=\Delta_{P}^{+}$. Its stalk cohomology is locally constant on $X_{P}$ and was shown to be

$$
H_{w}^{i}\left(\mathbf{B}^{\bullet}\right) \cong H^{j-\left|\Delta_{P}^{+}\right|}\left(\mathfrak{M}_{P}, \mathbf{E}\right)_{\left\lceil v_{P}, \Delta_{P}^{+}\right\rfloor} .
$$

Since $h_{2}$ is a smooth closed embedding we have a canonical quasi-isomorphism (12.3.1)

$$
\mathbf{C}^{\bullet}:=h_{2}^{!} \mathbf{B}^{\bullet} \cong h_{2}^{*}\left(\mathbf{B}^{\bullet}\right) \otimes \mathcal{O}[-c]
$$

where $c=\operatorname{dim}\left(X_{P}\right)-\operatorname{dim}\left(F^{\prime} \cap X_{P}\right)$ and where $\mathcal{O}$ is the orientation bundle (i.e. the top exterior power) of the normal bundle of $F^{\prime} \cap X_{P}$ in $X_{P}$. The complex $\mathbf{C}^{\bullet}$ is constructible with respect to the stratification of $\bar{X}$, meaning that its cohomology sheaves are locally constant on $X_{P}$, hence also on $E$. But $E$ is a manifold with boundary, so

$$
h_{1}^{!} \mathbf{C}^{\bullet} \cong i_{!} \mathbf{C}^{\bullet} \mid(E-\partial E)
$$

is obtained by first restricting to the interior $E-\partial E$ and then extending by 0 . (Here, $i: E-\partial E \rightarrow E$ denotes the inclusion.) Thus the cohomology of $h_{1}^{!} \mathbf{C}^{\bullet}$ is the compactly supported cohomology $H_{c}^{i}\left(E-\partial E ; \mathbf{C}^{\bullet}\right) \cong H_{c}^{i}\left(F^{\prime} ; \mathbf{C}^{\bullet}\right)$.

Next we must compute the pointwise Lefschetz number $n(w)$ for $w \in E$, that is, the alternating sum of the traces on the stalk cohomology of $\mathbf{A}^{\bullet}=h_{1}^{!} \mathbf{C}^{\bullet}$. By (13.10.2) it is 0 when $w \in \partial E$, so let $w \in E-\partial E$. Then

$$
\begin{aligned}
H_{w}^{i}\left(\mathbf{C}^{\bullet}\right) & =H_{w}^{i-c}\left(h_{2}^{*} \mathbf{B}^{\bullet} \otimes \mathcal{O}\right) \\
& =H^{i-c-\left|\Delta_{P}^{+}\right|}\left(\mathfrak{N}_{P}, E\right)_{\left\lceil v_{P}, \Delta_{P}^{+}\right\rfloor} \otimes \mathcal{O}_{w} .
\end{aligned}
$$

By $§ 8.6$, the mapping $c_{1}: F_{P}(e) \rightarrow F^{\prime}$ is a covering of degree 


$$
d_{e}=\left[\Gamma_{L} \cap \bar{y}^{-1} \Gamma_{L} \bar{y}: v_{P}\left(\Gamma_{P} \cap y^{-1} \Gamma_{P} y\right)\right] .
$$

Near each fixed point $w^{\prime} \in c_{1}^{-1}(w)$ the Hecke correspondence acts on the $\mathfrak{N}_{P}$-cohomology through the homomorphism $r \phi\left(e^{-1}\right)$ (using Lemma 13.8), and by $\S 8.7$ it acts on $\mathcal{O}_{w}$ by $(-1)^{c}$. Summing these contributions over the $d_{e}$ different points in $c_{1}^{-1}(w)$ gives

$$
\begin{aligned}
n(w) & =d_{e} r(-1)^{-c-\left|\Delta_{P}^{+}\right|}(-1)^{c} \sum_{i \geqq 0}(-1)^{i} \operatorname{Tr}\left(\phi\left(e^{-1}\right) ; H^{i}\left(\mathfrak{N}_{P}, E\right)_{\left[v_{P}, \Delta_{P}^{+}\right\rfloor}\right) \\
& =d_{e} r(-1)^{\left|\Delta_{P}^{+}\right|} \sum_{\substack{v \in W_{P}^{1} \\
I_{v}(v)=\Delta_{P}^{+}}}(-1)^{\ell(v)} \operatorname{Tr}\left(e^{-1} ; V_{v\left(\lambda_{B}+\rho_{B}\right)-\rho_{B}}^{L}\right)
\end{aligned}
$$

by Proposition 12.8. The contribution arising from $F_{P}(e)$ is this quantity times $\chi_{c}(E-\partial E)=\chi_{c}\left(F^{\prime}\right)$. However (by §8.6), $\chi_{c}\left(F_{P}(e)\right)=d_{e} \chi_{c}\left(F^{\prime}\right)$ which absorbs the factor of $d_{e}$ in (13.10.6) and therefore completes the proof of Theorem 13.6.

\section{Proof of Theorem 1.5}

14.1. As in $\S 2, \mathbf{G}$ denotes a connected reductive linear algebraic group defined over $\mathbb{Q}, D=G / K^{\prime}$ is its associated symmetric space with basepoint $x_{0} \in D$ and stabilizer $K^{\prime}=A_{G} K\left(x_{0}\right)$. Let $\Gamma \subset \mathbf{G}(\mathbb{Q})$ denote an arithmetic subgroup which we assume to be neat, and $X=\Gamma \backslash D$. Throughout this section we fix a Hecke correspondence $\left(c_{1}, c_{2}\right): \bar{C} \rightrightarrows \bar{X}$ defined by some element $g \in \mathbf{G}(\mathbb{Q})$. So $C=\Gamma^{\prime} \backslash D$ with $\Gamma^{\prime}=\Gamma \cap g^{-1} \Gamma g$. We also fix a $\Gamma$ equivariant tiling of $D$ which is narrow with respect to the Hecke correspondence. Choose $\mathbf{t} \in A_{P_{0}}(>1)$ in accordance with Proposition 11.2.

Let $F \subset \bar{C}$ denote the (full) fixed point set of the Hecke correspondence $\bar{C} \rightrightarrows \bar{X}$ and let $E$ denote the (full) fixed point set of the modified Hecke correspondence (11.1.1). Then

$$
F=\coprod_{\{\mathbf{P}\}} F \cap C_{P} \quad \text { and } \quad E=\coprod_{\{\mathbf{P}\}} F \cap C_{P}^{0}
$$

where the union is over the strata of $\bar{C}$, that is, over $\Gamma^{\prime}$-conjugacy classes of rational parabolic subgroups $\mathbf{P} \subseteq \mathbf{G}$. Each $F \cap C_{P}^{0}$ is a union of connected components of $E$ by Proposition 11.2. The Lefschetz fixed point theorem (Theorem 13.2) may be used to write the Lefschetz number as a sum over these individual strata.

14.2. Contribution from a single stratum. Let $\mathbf{P} \cong \mathbf{G}$ be a rational parabolic subgroup and suppose that $c_{1}\left(C_{P}\right)=c_{2}\left(C_{P}\right)=X_{P}$. By Proposition 7.3, in a neighborhood of $C_{P}$ the correspondence is isomorphic to the parabolic Hecke correspondence determined by some $y \in \Gamma g \Gamma \cap P$ and moreover (in this neighborhood) the fixed points of the modified correspondence coincide with those of $E \cap C_{P}=F \cap C_{P}^{0}$.

If $F_{P}(e)$ denotes the set of fixed points in $C_{P}$ with characteristic element $e \in \Gamma_{L} \bar{y} \Gamma_{L}$, then by Proposition 8.4,

$$
F \cap C_{P}=\coprod_{\{e\}} F_{P}(e) \text { and } \quad E \cap C_{P}=\coprod_{\{e\}} F_{P}(e) \cap C_{P}^{0}
$$


where the union is over $\Gamma_{L}$-conjugacy classes of elements $\{e\} \subset \Gamma_{L} \bar{y} \Gamma_{L}$ which are elliptic modulo $A_{P}$. (Here, $\Gamma_{L}=v_{P}(\Gamma \cap P) \subset L_{P}$ and $\bar{y}=v_{P}(y)$.) For each such conjugacy class $\{e\}$, the set $F_{P}(e)$ consists of finitely many connected components, say, $F_{1}, F_{1}, \ldots, F_{m}$. The contribution to the Lefschetz number from the component $F_{j}$ is given by Theorem 13.6. By (8.4.1) (see also $\$ 8.6, \S 15.8$ ),

$$
\sum_{j=1}^{m} \chi_{c}\left(F_{j}\right)=\chi_{c}\left(F_{P}(e)\right)=\chi_{c}\left(\Gamma_{e}^{\prime} \backslash L_{e} / K_{e}^{\prime}\right)
$$

So the contribution to the Lefschetz number from the stratum $C_{P} \rightrightarrows X_{P}$ is

$$
L(P, y):=\sum_{\{e\}} \chi_{c}\left(\Gamma_{e}^{\prime} \backslash L_{e} / K_{e}^{\prime}\right) r(-1)^{\left|\Delta_{P}^{+}\right|} \sum_{\substack{w \in W_{P}^{1} \\ I_{v}(w)=\Delta_{P}^{+}(e)}}(-1)^{\ell(w)} \operatorname{Tr}\left(e^{-1} ; V_{w\left(\lambda_{B}+\rho_{B}\right)-\rho_{B}}^{L}\right)
$$

where the index set for the first sum is the same as that for the union in (14.2.1). This quantity $L(P, y)$ depends only on the local system $\mathbf{E}$, the choice of parabolic subgroup $\mathbf{P}$ and the element $y \in P$.

14.3. Sum over strata. Let $\mathbf{P}_{1}, \mathbf{P}_{2}, \ldots, \mathbf{P}_{t}$ denote a collection of representatives, one from each $\Gamma$-conjugacy class of rational parabolic subgroups $\mathbf{P} \subseteq \mathbf{G}$. These index the strata of $\bar{X}$. For each such $i$ the intersection $\Gamma g \Gamma \cap P_{i}$ decomposes:

$$
\Gamma g \Gamma \cap P_{i}=\coprod_{j} \Gamma_{P_{i}} y_{i j} \Gamma_{P_{i}}
$$

Lemma 7.4 gives a one-to-one correspondence between this collection $\left\{y_{i j}\right\}$ and strata $C_{i j}$ of $\bar{C}$ such that $c_{1}\left(C_{i j}\right)=c_{2}\left(C_{i j}\right)$. Moreover the restriction of the Hecke correspondence to a neighborhood of $C_{i j}$ is locally isomorphic to the parabolic Hecke correspondence defined by $y_{i j}$ so the local contribution to the Lefschetz number from $C_{i j}$ equals the number $L\left(P_{i}, y_{i j}\right)$ given in (14.2.2). In summary, the total Lefschetz number is

$$
L(g)=\sum_{i=1}^{t} \sum_{j} L\left(P_{i}, y_{i j}\right)
$$

as claimed in Theorem 1.5.

14.4. Another formula. If a little expansion $S h(t)^{-1}$ is used instead of the shrink, this will convert neutral directions normal to each stratum into expanding directions, and it will convert the tangential distance into a contracting direction. An indicator mapping replacing $(13.10 .1)$ is

$$
t(x)=\left(\sum_{\alpha \in \Delta_{P}^{+} \cup \Delta_{P}^{0}} r_{\alpha}^{P}(x), \sum_{\alpha \in \Delta_{P}^{-}} r_{\alpha}^{P}(x)+d_{E} \pi_{P}(x)\right) .
$$

This changes the nature of the sheaf $\mathbf{A}^{\bullet}$ with the result that the Euler characteristic (rather than the Euler characteristic with compact supports) appears in the formula. So, in equa- 
tion (14.3.1), the contribution $L(P, y)(14.2 .2)$ from the stratum $C_{P} \rightrightarrows X_{P}$ will be replaced by the quantity

$$
L^{\prime}(P, y)=\sum_{\{e\}} r(-1)^{\left|\Delta_{P}^{+} \cup \Delta_{P}^{0}\right|} \chi\left(\Gamma_{e}^{\prime} \backslash L_{e} / K_{e}^{\prime}\right) \sum_{\substack{w \in W_{P}^{1} \\ I_{v}(w)=\Delta_{P}^{+} \cup \Delta_{P}^{0}}}(-1)^{\ell(w)} \operatorname{Tr}\left(e^{-1} ; V_{w\left(\lambda_{B}+\rho_{B}\right)-\rho_{B}}^{L}\right)
$$

where the summations are over the same index sets as in (14.2.2), and where $\Delta_{P}^{+}=\Delta_{P}^{+}(e)$ and $\Delta_{P}^{0}=\Delta_{P}^{0}(e)$.

\section{Remarks on the Euler characteristic}

As in $\S 2, \mathbf{G}$ denotes a connected linear reductive algebraic group defined over $\mathbb{Q} ; D$ denotes the associated symmetric space; $\mathbf{S}_{\mathbf{G}}$ denotes the greatest $\mathbb{Q}$-split torus in the center of $G ; A_{G}=\mathbf{S}_{\mathbf{G}}(\mathbb{R})^{0}$ denotes the identity component of its real points; $K^{\prime}=A_{G} K$ is the stabilizer in $G$ of a fixed basepoint $x_{0} \in D ; \Gamma \subset \mathbf{G}(\mathbb{Q})$ is an arithmetic group, and $X=\Gamma \backslash D$.

15.1. Proposition. Suppose $\left(\mathbf{G} / \mathbf{S}_{\mathbf{G}}\right)(\mathbb{R})$ does not contain a compact maximal torus. Then $\chi(X)=\chi_{c}(X)=0$, that is, both the Euler characteristic and the Euler characteristic with compact supports vanish.

The proof will appear in $§ 15.6$.

15.2. Lemma. Let $X=\Gamma \backslash G / K A_{G}$. Then the Euler characteristic and the Euler characteristic with compact supports coincide: $\chi(X)=\chi_{c}(X)$.

15.3. Proof. Let $\tilde{X}$ denote the Borel-Serre compactification of $X$. Topologically, it is a manifold with boundary $\partial \tilde{X}=\tilde{X}-X$. Since $H_{c}^{i}(X)=H^{i}(\tilde{X}, \partial \tilde{X})$, it suffices to show that $\chi(\partial \tilde{X})=0$. The boundary $\partial \tilde{X}$ is a union of finitely many boundary strata $Y_{P}$, each of which fibers over the corresponding stratum $X_{P}$ (of the reductive Borel-Serre compactification) with fiber a nilmanifold $N_{P}$ (cf. $\left.\S 2.5,13.7\right)$. So $\chi\left(Y_{P}\right)=\chi\left(N_{P}\right) \chi\left(X_{P}\right)=0$. It follows from Mayer-Vietoris that $\chi(\partial \tilde{X})=0$.

For completeness we also include a proof of the following often-cited fact.

15.4. Lemma. Suppose the real Lie group $G / A_{G}$ does not contain a compact maximal torus. Then the Euler form vanishes identically on $X$.

15.5. Proof. By replacing $\mathbf{G}$ by the algebraic group ${ }^{\mathbf{0}} \mathbf{G}$ (and noting that $\left.X=\Gamma \backslash{ }^{0} G / K\right)$, we may assume that $\mathbf{S}_{\mathbf{G}}$ is trivial. Let $\mathfrak{g}=\mathfrak{f} \oplus \mathfrak{p}$ be the Cartan decomposition of $\operatorname{Lie}(G)$ corresponding to the choice $K$ of maximal compact subgroup. Choose a $K$ invariant inner product on $\mathfrak{p}$. This determines a $G$-invariant Riemannian metric on $D=G / K$ which passes to a Riemannian metric on $X$. Let $\Omega$ be the curvature form of the torsion-free Levi-Civita connection which is associated to this metric. The resulting Euler form $E u$ is defined to be 0 if $\operatorname{dim}(D)$ is odd. If $\operatorname{dim}(D)=2 k$ then $E u$ is the $G$-invariant differential form on $D$ whose value at the basepoint $x_{0}$ is 


$$
E u\left(\dot{x}_{1}, \dot{y}_{1}, \dot{x}_{2}, \dot{y}_{2}, \ldots, \dot{x}_{k}, \dot{y}_{k}\right)=P\left(\Omega\left(\dot{x}_{1}, \dot{y}_{1}\right), \ldots, \Omega\left(\dot{x}_{k}, \dot{y}_{k}\right)\right)
$$

(for any $\dot{x}_{1}, \ldots, \dot{y}_{k} \in \mathfrak{p}=T_{x_{0}} D$ ), where $P$ is the polarization of the Pfaffian Pf $: \operatorname{End}(\mathfrak{p})^{-} \rightarrow \mathbb{R}$. (Here, $\operatorname{End}(\mathfrak{p})^{-}$denotes the skew-adjoint endomorphisms of $\mathfrak{p}$.) The form $E u$ on $D$ passes to a differential form on $X=\Gamma \backslash D$, which is the Euler form for $X$.

Let $\mathrm{Ad}: K \rightarrow G L(\mathfrak{p})$ be the adjoint representation and let ad $: \mathfrak{f} \rightarrow \operatorname{End}(\mathfrak{p})^{-}$be its derivative. We claim that $\operatorname{det}(\operatorname{ad}(\dot{k}))=0$ for any $\dot{k} \in \mathfrak{f}$. Modify $\dot{k}$ by conjugacy if necessary, so as to guarantee that $\dot{k}$ lies in a maximal torus $\mathrm{t} \subset \mathfrak{g}$ which is stable under the Cartan involution $([\mathrm{Wa}], \S 1.2,1.3)$. Then $\mathrm{t}=\mathrm{t}_{+} \oplus \mathrm{t}_{-}$with $\mathrm{t}_{+} \subset \mathfrak{f}$ and $\mathrm{t}_{-} \subset \mathfrak{p}$. By assumption, $\mathrm{t}_{-}$ contains a nonzero vector $\dot{t}$, and $\operatorname{ad}(\dot{k})(\dot{t})=[\dot{k}, \dot{t}]=0$, which proves the claim.

The principal $K$-bundle $G \rightarrow D=G / K$ admits a canonical $G$-invariant connection ([KN], Chapt. II, Thm. 11.5). Its curvature form $\omega \in \mathscr{A}^{2}(D, \mathfrak{l})$ is the $G$-invariant differential form whose value at the basepoint $x_{0}$ is given by $\omega_{0}\left(\dot{p}_{1}, \dot{p}_{2}\right)=-\left[\dot{p}_{1}, \dot{p}_{2}\right] \in \mathfrak{f}$ for any $\dot{p}_{1}, \dot{p}_{2} \in \mathfrak{p}$. By a theorem of Nomizu [No2], for any real representation $\lambda: K \rightarrow G L(E)$, the resulting connection in the associated $G$-homogeneous vector bundle $\mathbf{E}=G \times_{K} E$ coincides with the torsion-free metric (Levi-Civita) connection of any $G$-invariant metric on $E$. Its curvature is the $G$-invariant $\operatorname{End}(\mathbf{E})$-valued differential form whose value at the basepoint is $\Omega_{0}=\lambda^{\prime} \circ \omega_{0}$ where $\lambda^{\prime}: \mathfrak{f} \rightarrow \operatorname{End}(E)$ is the differential of $\lambda$. Taking $\lambda=\operatorname{Ad}: K \rightarrow G L(\mathfrak{p})$ as above gives $\Omega_{0}\left(\dot{p}_{1}, \dot{p}_{2}\right)=-\operatorname{ad}\left(\left[\dot{p}_{1}, \dot{p}_{2}\right]\right)$. By the above claim, this has determinant 0 hence its Pfaffian vanishes also. Therefore the Euler form is zero on $D$, so it is also zero on $X$.

15.6. The proof of Proposition 15.1 is then a consequence of the following classical result of Harder $[\mathrm{H}]$ (a more streamlined proof of which may be found in [Le2]).

15.7. Theorem. The Euler characteristic $\chi(X)$ is given by the integral over $X$ of the Euler form with respect to any invariant Riemannian metric on $X$.

15.8. Euler characteristic of a fixed point component. Now suppose that $X_{P} \subset \bar{X}$ is a boundary stratum corresponding to a rational parabolic subgroup $P=\mathscr{U}_{P} L_{P}$. Let $F_{P}(e) \subset X_{P}$ be the set of fixed points with some fixed (elliptic) characteristic element $e \in \mathbf{L}_{\mathbf{P}}(\mathbb{Q})$. Let $L_{e}$ be the centralizer of $e$ in $L_{P}$. By $(8.4 .1), F_{P}(e) \cong \Gamma_{e}^{\prime} \backslash L_{e} / K_{e}^{\prime}$ where $\Gamma_{e}^{\prime}=\Gamma_{L}^{\prime} \cap L_{e}$ and where $K_{e}^{\prime}=L_{e} \cap\left(z\left(K_{P} A_{P}\right) z^{-1}\right.$ ) (for appropriate $z$ ). By (8.4.2),

$$
\chi_{c}\left(\Gamma_{e}^{\prime} \backslash L_{e} / K_{e}^{\prime}\right)=d_{e} \chi_{c}\left(\Gamma_{e} \backslash L_{e} / K_{e}^{\prime}\right)
$$

where $\Gamma_{e}=\Gamma_{L} \cap L_{e}$ and $d_{e}=\left[\Gamma_{e}: \Gamma_{e}^{\prime}\right]$. This expression has the following merit. The contribution (14.2.2) to the Lefschetz number from the stratum $C_{P}$ depends on the subgroup $\Gamma_{P}^{\prime} \subset \Gamma_{P}$. However once this expression (15.8.1) has been substituted into (14.2.2), the dependency on this subgroup $\Gamma_{P}^{\prime}$ occurs only in the two integers $r$ and $d_{e}$.

15.9. Descent. Let $\mathbf{S}_{e}$ be the greatest $\mathbb{Q}$-split torus in the center of $L_{e}$ and let $A_{e}$ be the identity component of its group of real points. As explained in $[\mathrm{GKM}], \S 7.11$, the group $K_{e}^{\prime}$ does not necessarily contain $\mathbf{S}_{\mathbf{e}}(\mathbb{R})$, so although $F_{P}(e)$ is not necessarily a "locally sym- 
metric space" in the sense of $\S 2$, it fibers over the locally symmetric space $\Gamma_{e}^{\prime} \backslash L_{e} / K_{e}^{\prime} A_{e}$ with fiber $A_{e} / A_{P}$ which is diffeomorphic to a Euclidean space. Therefore

$$
\chi_{c}\left(F_{P}(e)\right)=(-1)^{\operatorname{dim}\left(A_{e} / A_{P}\right)} \chi_{c}\left(\Gamma_{e}^{\prime} \backslash L_{e} / K_{e} A_{e}\right)=(-1)^{\operatorname{dim}\left(A_{e} / A_{P}\right)} d \chi_{c}\left(\Gamma_{e} \backslash L_{e} / K_{e} A_{e}\right)
$$

(where $K_{e}=L_{e} \cap\left(z K_{P} z^{-1}\right)$ ).

Now suppose that $L_{P} / A_{P}$ does not contain a compact maximal torus. According to the preceding remarks, $\chi_{c}\left(C_{P}\right)=\chi_{c}\left(X_{P}\right)=0$. However the contribution (14.2.2) to the Lefschetz number from the stratum $C_{P}$ does not necessarily vanish. Assuming $L_{P} / A_{P}$ does not contain a compact maximal torus, the same will be true of $L_{e} / A_{P}$. If, moroever, $A_{P}=A_{e}$ then $\chi_{c}\left(F_{P}(e)\right)=0$. However if $A_{P}$ differs from $A_{e}$, it is possible that $\chi\left(F_{P}(e)\right) \neq 0$ (in which case $F_{P}(e)$ is necessarily non-compact since it is fibered by $A_{e} / A_{P}$ as described above). See Example 16.4 in which $L_{e}=A_{e}$ and $F_{P}(e) \cong A_{e} / A_{P}$ is the orbit of a split torus. In such cases it is possible to re-attribute the contribution (14.2.2) from the stratum $C_{P} \rightrightarrows X_{P}$ to smaller strata in the correspondence. This procedure is carried out in [GKM], p. 531, resulting in a Lefschetz formula in which the only nonzero contributions come from strata $C_{P} \rightrightarrows X_{P}$ such that $L_{P}$ has a compact maximal torus.

15.10. In the adelic setting, the Euler characteristic with compact support $\chi_{c}\left(F_{P}(e)\right)$ can be expressed in terms of orbital integrals (cf. [GKM], §7.11 and §7.14).

\section{Examples and special cases}

16.1. Reducible fixed point components. For $G=S L(3, \mathbb{R}), \quad D=G / K$, and $\Gamma \subset S L(3, \mathbb{Z})$ a neat principal congruence subgroup, the reductive Borel-Serre compactification $\bar{X}$ contains a singular 0-dimensional stratum $X_{B}$ corresponding to the standard Borel subgroup B. This stratum is contained in the closures of the strata $X_{P_{1}}$ and $X_{P_{2}}$ corresponding to the standard maximal parabolic subgroups $\mathbf{P}_{\mathbf{1}}, \mathbf{P}_{\mathbf{2}}$ containing $\mathbf{B}$. Let $g$ be a generic element of $\mathbf{G}(\mathbb{Q}) \cap \mathscr{U}\left(P_{1}\right) \cap \mathscr{U}\left(P_{2}\right)$ which is not in $\Gamma$, for example,

$$
g=\left(\begin{array}{ccc}
1 & 0 & 1 / 2 \\
0 & 1 & 0 \\
0 & 0 & 1
\end{array}\right)
$$

Let $\bar{C} \rightrightarrows \bar{X}$ be the resulting Hecke correspondence. Then these three strata $X_{P_{1}}, X_{P_{2}}$, and $X_{B}$ are fixed by the correspondence. However points in $X$ which are sufficiently close to these strata are not fixed.

16.2. Middle weight for $\mathbf{S p}_{4}$. Let $\mathbf{G}=\mathbf{S p}_{4}$, fix a neat arithmetic subgroup $\Gamma \subset \mathbf{G}(\mathbb{Q})$, and choose a Hecke correspondence $\bar{C} \rightrightarrows \bar{X}$ which is determined by some $g \in \mathbf{G}(\mathbb{Q})$. If $\mathbf{P}$ is a minimal parabolic subgroup of $\mathbf{G}$ then its Levi quotient $\mathbf{L}_{\mathbf{P}}=\mathbf{S}_{\mathbf{P}}$ is a maximal split torus and the boundary stratum $C_{P}$ consists of a single point. Suppose this point is an isolated fixed point of the Hecke correspondence. Let $e$ be its characteristic element. The vector 
space $\chi_{\mathbb{Q}}^{*}\left(\mathbf{S}_{\mathbf{P}}\right)$ has a basis consisting of the simple roots $\Delta_{P}=\{\alpha, \beta\}$. Let $\left\{t_{\alpha}, t_{\beta}\right\}$ be the dual basis of $\chi_{*}^{\mathbb{Q}}\left(\mathbf{S}_{\mathbf{P}}\right)$, so that $\left\langle\beta, t_{\beta}\right\rangle=1,\left\langle\beta, t_{\alpha}\right\rangle=0$ and the same with $\alpha$ and $\beta$ interchanged. See Figure 6.

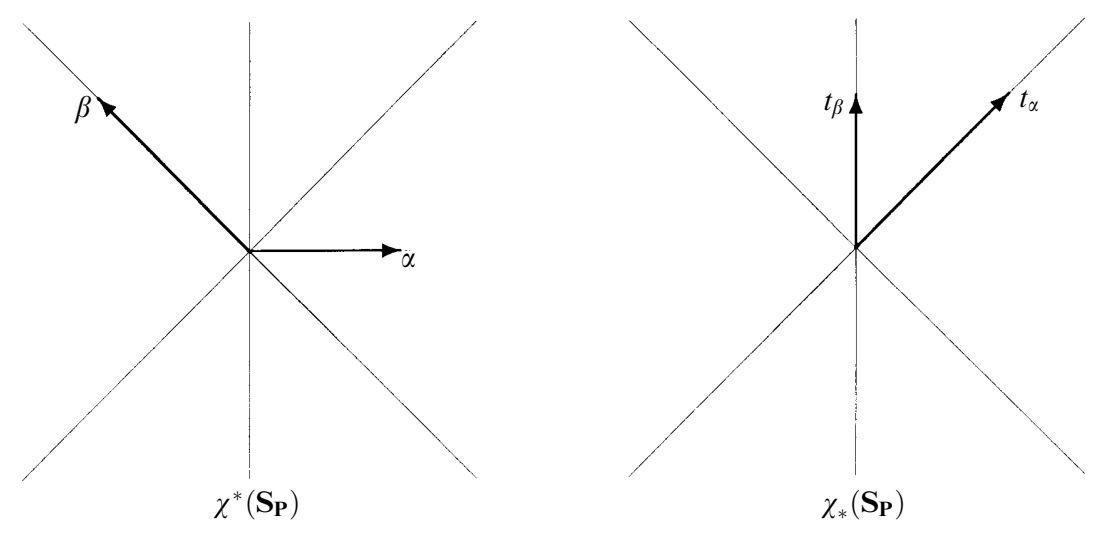

Figure 6. Simple roots and dual basis.

Let us take $\mathbf{E}$ to be the trivial local system, and the weight profile $v=-\rho_{B}$ to be the middle weight (where $\rho_{B}$ is the half-sum of the positive roots). The cohomology $H^{*}\left(\mathfrak{M}_{P}, \mathbb{C}\right)$ decomposes into a sum of 1-dimensional weight spaces,

$$
V_{w \rho_{B}-\rho_{B}} \subset H^{\ell(w)}\left(\mathfrak{N}_{P}, \mathbb{C}\right)
$$

as $w \in W$ varies over the elements of the full Weyl group. These weights are the dots in the left hand part of Figure 7, in which the origin is at $-\rho_{B}$. For each weight space indexed by a given $w \in W$ we have indicated the corresponding set

$$
I_{v}(w)=\left\{\theta \in \Delta_{P} \mid\left\langle w \rho_{B}, t_{\theta}\right\rangle<0\right\}
$$

of simple roots. The cohomology $H^{*}\left(\mathfrak{\Re}_{P}\right)$ is divided into four "quadrants" according to the value of $I_{v}(w)$.

If necessary, project the characteristic element $e$ to the identity component $A_{P}$ of the torus $\mathbf{S}_{\mathbf{P}}(\mathbb{R})$ and let $t \in \mathfrak{A}_{P}=\operatorname{Lie}\left(A_{P}\right)$ denote its log. The right hand half of Figure 7 may be identified with the Lie algebra $\mathfrak{A}_{P}$. The chamber containing $t$ determines the expandingcontracting nature of the Hecke correspondence near this fixed point. In each chamber we have indicated the set of expanding roots,

$$
\Delta_{P}^{+}(e)=\left\{\theta \in \Delta_{P} \mid \theta(t)<0\right\}
$$

(where now $\theta \in \Delta_{P}$ has been identified with a homomorphism $\mathfrak{A}_{P} \rightarrow \mathbb{R}$ ). The Lie algebra $\mathfrak{A}_{P}$ is divided into four "quadrants" according to the value of $\Delta_{P}^{+}(e)$ (although we have not indicated which quadrant contains a given "wall"). 

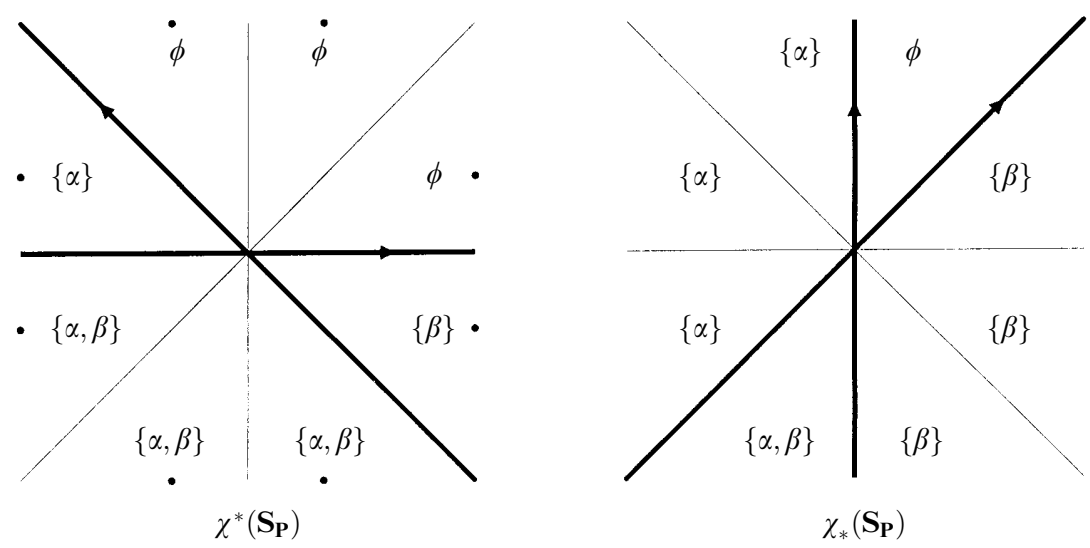

Figure 7. Diagram of $I_{v}(w)$ and of $\Delta_{P}^{+}(e)$.

Theorem 13.6 states that the portion of $H^{*}\left(\mathfrak{N}_{P}\right)$ which contributes to the Lefschetz number at this fixed point depends on the quadrant in which $t=\log (e)$ lies: if $\Delta_{P}^{+}(e)=J \subset \Delta_{P}$ then only the portion of $H^{*}\left(\mathfrak{N}_{P}\right)$ which lies in the quadrant indexed by $J$ contributes to the Lefschetz number. A further degree shift by $|J|$ occurs when this portion $H^{*}\left(\mathfrak{N}_{P}\right)_{\left\lceil v_{P}, J\right\rfloor}$ is identified (in Theorem 12.5) with the local weighted cohomology with supports.

It is a remarkable fact that, globally in the Hecke correspondence, the fixed points occur in Weyl group orbits. Assuming $t$ is regular (does not lie on a wall) then, after summing over all the fixed points, each chamber will appear the same number of times. It is the sum of these local contributions over a $W$-orbit of fixed points ([GKM], p. 529, last paragraph) which gives rise to the combinatorial formula for the averaged discrete series characters as described in $[\mathrm{GKM}]$.

16.3. Very positive and very negative weights. Let $i: X \rightarrow \bar{X}$ denote the inclusion. Suppose the weight profile $v=-\infty$ (or is very negative). Then the weight truncation does nothing, and the weighted cohomology sheaf $\mathbf{W}^{v} \mathbf{C}^{\bullet}(\mathbf{E}) \cong R i_{*}(\mathbf{E})$ becomes the "full" direct image of E. For any stratum $X_{Q}, I_{v}(w)=\emptyset$ for any $w \in W_{Q}^{1}$. Theorem 13.6 then says that a fixed point stratum $F \cap C_{Q}$ (with characteristic element $e$ ) makes a contribution to the Lefschetz number only if $\Delta_{Q}^{+}(e)=\emptyset$, which is to say, only if the Hecke correspondence is either contracting or neutral in every direction normal to the stratum $X_{Q}$.

In this case the local contribution to the Lefschetz number may be expressed in terms of the character of the finite dimensional representation $G \rightarrow G L(E)$. We briefly recall the argument in $[\mathrm{GKM}], \S 7.18$. The quantity $\sum_{i}(-1)^{i} \operatorname{Tr}\left(e^{-1} ; H^{i}\left(\mathfrak{N}_{P}, E\right)\right)$ is equal to $\operatorname{Tr}\left(e^{-1} ; E\right)$
times the following quantity:

$$
\begin{aligned}
\sum_{i}(-1)^{i} \operatorname{Tr}\left(e^{-1} ; \wedge^{i}\left(\mathfrak{N}_{P}^{*}\right)\right) & =\operatorname{det}\left(1-\operatorname{Ad}(e) ; \mathfrak{N}_{P}(\mathbb{C})\right) \\
& =\prod_{\alpha \in \Phi_{L}^{+}}\left(1-\alpha^{-1}(e)\right) \prod_{\alpha \in \Phi_{L}^{+}} \alpha(e)(-1)^{\operatorname{dim} \mathfrak{N}_{P}} \\
& =\Delta_{P}(e) \operatorname{det}\left(e ; \mathfrak{N}_{P}\right)(-1)^{\operatorname{dim} \mathfrak{N}_{P}}
\end{aligned}
$$


where $\Delta_{P}(e)=\prod_{\alpha \in \Phi_{L}^{+}}\left(1-\alpha^{-1}(e)\right)$ denotes the (partial) Weyl denominator. (These quantities may be further expressed in terms of $\left|D_{L}^{G}(e)\right|, \delta_{P}(e)$, and $\chi_{G}(e)$ using [GKM], (7.16.11), (7.18.3) and [GKM], p. 497.)

Similarly, suppose the weight profile is $v=+\infty$ or is a very large positive weight. The stalk cohomology (at a point $x \in X_{Q}$ in some boundary stratum $X_{Q}$ ) of the weighted cohomology sheaf $\mathbf{W}^{v} \mathbf{C}^{\bullet}(\mathbf{E})$ vanishes because the weight truncation (12.2.1) kills everything. In this case, the weighted cohomology sheaf is quasi-isomorphic to the sheaf $R i_{!}(\mathbf{E})$ which is obtained as the extension by 0 of the local system $\mathbf{E}$. Its cohomology is the compact support cohomology $H_{c}^{*}(X ; \mathbf{E})$ of the locally symmetric space. For any stratum $X_{Q}$, according to (12.7.2), $I_{v}(w)=\Delta_{Q}$ for any $w \in W_{Q}^{1}$. Theorem 13.6 then says that a fixed point stratum $F \cap C_{Q}$ (with characteristic element $e \in L_{Q}$ ) makes a contribution to the Lefschetz number only if $\Delta_{Q}^{+}(e)=\Delta_{Q}$, that is, only if the Hecke correspondence is strictly expanding in all directions normal to the stratum $X_{Q}$. Then the same quantity $\sum_{i}(-1)^{i} \operatorname{Tr}\left(e^{-1} ; H^{i}\left(\mathfrak{N}_{P}, E\right)\right)$ occurs in the formula, but with a (possibly) different sign.

In these cases (of $v= \pm \infty$ ) the Lefschetz formula of Franke [F] can be recovered, cf. [GKM], §7.17, 7.18.

16.4. Hyperbolic 3-space. For $\mathbf{G}(\mathbb{R})=S L_{2}(\mathbb{C})$ the symmetric space $D=G / K$ may be identified with hyperbolic 3-space. If $\Gamma$ is a torsion-free arithmetic group, then $X=\Gamma \backslash D$ is a hyperbolic 3-manifold. The group $G$ does not contain a compact maximal torus. Consequently, $\chi(X)=0$ (cf. §15.1). However, when $X$ is not compact, there exists a Hecke correspondence on $X$ whose fixed point set consists of a smooth curve which passes from one cusp to another cusp. The Euler characteristic of this fixed point set is not zero, although the Euler form vanishes identically. The fixed point set is not a "locally symmetric space" in the sense of $\$ 2.1$ because it contains (and in fact consists of) a Euclidean factor, cf. §15.9. It is possible to find particular weight profiles such that the (global) Lefschetz number of this correspondence on the weighted cohomology is nonzero. However, the formula $[\mathrm{GKM}]$ (thm. 7.14.B) would attribute the contribution from this fixed curve to the cusps, rather than to the interior stratum. This re-attribution is a result of equation (7.14.2) of $[\mathrm{GKM}]$.

16.5. Nielsen fixed point theory. Suppose $X$ is a compact manifold with fundamental group $\Gamma=\pi_{1}\left(X, x_{0}\right)$. Let $f: X \rightarrow X$ be a self-map. A choice of path from the basepoint $x_{0}$ to its image $f\left(x_{0}\right)$ determines a homomorphism $\phi: \Gamma \rightarrow \Gamma$. Two elements $\gamma_{1}, \gamma_{2} \in \Gamma$ are said to be $\phi$-conjugate if there exists $\gamma \in \Gamma$ so that $\gamma_{2}=\gamma \gamma_{1} \phi(\gamma)^{-1}$. Let $(\Gamma)_{\phi}$ denote the set of $\phi$-conjugacy classes in $\Gamma$ and let $\mathbb{R}(\Gamma)_{\phi}$ be the vector space of finite formal linear combinations of such classes. For each connected component $F$ of the fixed point set of $f$, let $L(F) \in \mathbb{R}$ denote the contribution of $F$ to the Lefschetz number $L$, that is,

$$
L=\sum_{i}(-1)^{i} \operatorname{Tr}\left(f^{*}: H^{i}(X) \rightarrow H^{i}(X)\right)=\sum_{F} L(F)
$$

The Nielsen theory (see $[\mathrm{GN}]$ ) assigns

- a $\phi$-conjugacy class $\{F\} \in(\Gamma)_{\phi}$ to each connected component $F$ of the fixed point set, and 
- a (cohomologically defined) Nielsen number $N(\{\gamma\}, f)$ to each $\phi$-conjugacy class $\{\gamma\}$ such that

$$
\sum_{\{\gamma\} \in(\Gamma)_{\phi}} N(\{\gamma\}, f)\{\gamma\}=\sum_{F} L(F)\{F\} \in \mathbb{R}(\Gamma)_{\phi}
$$

thereby "refining" the Lefschetz fixed point formula. (The sum on the left is over $\phi$ conjugacy classes and the sum on the right is over connected components of the fixed point set.)

Now suppose that $X=\Gamma \backslash D$ is a compact locally symmetric space. Fix $g \in \mathbf{G}(\mathbb{Q})$ and let $C \rightrightarrows X$ be the resulting Hecke correspondence. Let $(\Gamma g \Gamma)_{1}$ be the set of $\Gamma$-conjugacy classes of elements $e \in \Gamma g \Gamma$. Let $\mathbf{E}$ be the local system corresponding to a representation $\tau: G \rightarrow G L(E)$. Theorem 1.5 then says that the Lefschetz number of this correspondence is:

$$
L=\sum_{\{e\}} \chi(F(e)) \operatorname{tr}\left(\tau(e)^{-1} ; E\right)
$$

Here, the sum is taken over all conjugacy classes $\{e\} \in(\Gamma g \Gamma)_{1}$, and $F(e)$ denotes the set of fixed points which have characteristic element equal to $e$. This set is empty unless $e$ is elliptic (modulo $A_{G}$ ). If $F(e)$ is not empty, then it is compact.

It turns out that if the local system $\mathbf{E}$ is trivial, and if the correspondence $C \rightrightarrows X$ is actually a self-map $f: X \rightarrow X$ then the terms in (16.5.2) are exactly the terms in the Nielsen formula (16.5.1). The group $\Gamma$ may be identified with the fundamental group $\pi_{1}\left(X, x_{0}\right)$. The Hecke correspondence is actually a self-map iff the element $g$ normalizes $\Gamma$. In this case, the automorphism $\phi: \Gamma \rightarrow \Gamma$ is given by conjugation: $\phi(\gamma)=g \gamma g^{-1}$. Finally, the association $a \mapsto a g$ (for $a \in \Gamma$ ) determines a one-to-one correspondence

$$
(\Gamma)_{\phi} \rightarrow(\Gamma g \Gamma)_{1}
$$

There is a slightly more general Nielsen formula for correspondences, also with coefficients in a local system. The terms in this formula again coincide with the terms in the sum (16.5.2).

\section{References}

[Ar1] J. Arthur, The $L^{2}$ Lefschetz numbers of Hecke operators, Inv. Math. 97 (1989), 257-290.

[Ar2] J. Arthur, A trace formula for reductive groups I, Duke Math. J. 45 (1978), 911-952.

[Ar3] J. Arthur, Characters, harmonic analysis, and an $L^{2}$-Lefschetz formula, The Mathematical Heritage of Hermann Weyl, Proc. Symp. Pure Math. 48, Amer. Math. Soc., Providence, RI, 1998.

[AMRT] A. Ash, D. Mumford, M. Rapoport, and Y.-S. Tai, Smooth Compactification of Locally Symmetric Varieties, Math. Sci. Press, Brookline, Mass., 1975.

[BaM] D. Barbasch and H. Moscovici, $L^{2}$-index and the Selberg trace formula, J. Funct. Anal. 53 (1983), 151-201.

[Be] J. Bewersdorff, Eine Lefschetzsche Fixpunktformel für Hecke-Operatoren, Bonner Math. Schr. 164, Univ. Bonn, 1985.

[Bo3] A. Borel, Linear Algebraic Groups (second edition), Grad. Texts Math. 126, Springer Verlag, New York 1991.

[Bo4] A. Borel, Stable real cohomology of arithmetic groups, Ann. Sci. Éc. Norm. Sup. 7 (1974), $235-272$. 
[Bo5] A. Borel, Sheaf theoretic intersection cohomology, Seminar on Intersection Cohomology, Progr. Math. 50, Birkhäuser, Boston 1984.

[BS] A. Borel and J.-P. Serre, Corners and Arithmetic Groups, Comm. Math. Helv. 48 (1973), $436-491$.

[E] W. T. van Est, A generalization of the Cartan-Leray sequence, i, ii, Indag. Math. XX (1958), $399-413$.

[F] J. Franke, Harmonic analysis in weighted $L_{2}$-spaces, Ann. Sci. Éc. Norm. Sup. 31 (1998), 181-279.

[GeM] S. Gelfand and Y. Manin, Homological Algebra, in: Algebra V, Encycl. Math. Sci. 38, Springer-Verlag, Berlin 1994.

[Gi] C. Gibson, K. Wirthmüller, A. Du Plessis, E. Looijenga, Topological Stability of Smooth Mappings, Lect. Notes Math. 552, Springer Verlag, New York 1976.

[GN] R. Geoghegan and A. Nicas, Parametrized Lefschetz-Nielsen fixed point theory and Hochschild homology traces, Amer. J. Math 116 (1994), 397-446.

[GHM] M. Goresky, G. Harder, and R. MacPherson, Weighted cohomology, Inv. Math. 116 (1994), $139-213$.

[GKM] M. Goresky, R. Kottwitz, and R. MacPherson, Discrete series characters and the Lefschetz formula for Hecke operators, Duke Math. J. 89 (1997), 477-554.

[GM1] M. Goresky and R. MacPherson, Lefschetz numbers of Hecke correspondences, in: The Zeta Functions of Picard Modular Surfaces, R. Langlands and D. Ramakrishnan, eds., Publ. C.R.M., Univ. de Montréal (1992), 465-478.

[GM2] M. Goresky and R. MacPherson, Local contribution to the Lefschetz fixed point formula, Inv. Math. 111 (1993), 1-33.

[GM3] M. Goresky and R. MacPherson, Stratified Morse Theory, Ergeb. Math. 14, Springer Verlag, Heidelberg 1989.

[GM4] M. Goresky and R. MacPherson, Intersection homology II, Inv. Math. 71 (1983), 77-129.

[GM5] M. Goresky and R. MacPherson, Errata to: Local contribution to the Lefschetz fixed point formula, 2003, http://arxiv.org/math.AT.0301289.

[GI] A. Grothendieck, L. Illusie, Formule de Lefschetz, S.G.A. 5, Cohomologie $l$-adique et Fonctions L, Lect. Notes Math. 589, Springer Verlag, Heidelberg (1977), 73-137.

$[\mathrm{H}] \quad$ G. Harder, Anhang (Letter to Goresky and MacPherson) in Eisensteinkohomologie und die Konstruktion gemischter Motive, Lect. Notes Math. 1562, Springer Verlag, Heidelberg 1993.

[I] B. Iverson, Cohomology of Sheaves, Universitext, Springer-Verlag, Heidelberg 1986.

[KS] M. Kashiwara and P. Schapira, Sheaves on Manifolds, Grundl. Math. Wiss. 292, Springer Verlag, Heidelberg 1990.

[KN] S. Kobayashi and K. Nomizu, Foundations of Differential Geometry I, II, John Wiley and Sons, New York 1966.

[Ko] B. Kostant, Lie algebra cohomology and the generalized Borel-Weil theorem, Ann. Math. 74 (1961), 329-387.

[Ku] M. Kuga and J. H. Sampson, A coincidence formula for locally symmetric spaces, Amer. J. Math 94 (1972), 486-500.

[L1] R. Langlands, Modular forms and $\ell$-adic representations, in: Modular Functions of One Variable II, Lect. Notes Math. 349, Springer Verlag, New York 1973.

[L2] R. Langlands, On the functional equation satisfied by Eisenstein series, Lect. Notes Math. 544, Springer Verlag, New York 1976.

[Le1] E. Leuzinger, An exhaustion of locally symmetric spaces by compact manifolds with corners, Inv. Math. 121 (1995), 389-410.

[Le2] E. Leuzinger, On the Gauss-Bonnet formula for locally symmetric spaces of noncompact type, Enseign. Math. 42 (1996), 201-214.

[Lo] E. Looijenga, $L^{2}$-cohomology of locally symmetric varieties, Comp. Math. 67 (1988), 3-20.

[Mac] R. MacPherson, Chern classes for singular varieties, Ann. Math. 100 (1974), 423-432.

[Mat] J. Mather, Notes on Topological Stability, mimeographed notes, Harvard University, 1970.

[Mo] H. Moscovici, Lefschetz formula for Hecke operators, Lie Groups and Representations III, Lect. Notes Math. 1077, Springer Verlag, New York (1984), 321-258.

[N] A. Nair, Weighted cohomology of locally symmetric spaces, Ann. Math. 150 (1999), 1-31.

[No1] K. Nomizu, On the cohomology of compact homogeneous spaces of nilpotent Lie groups, Ann. Math. 59 (1954), 531-538.

[No2] K. Nomizu, Invariant affine connections on homogeneous spaces, Amer. J. Math. 76 (1954), $33-65$.

[R] M. Rapoport, On the shape of the contribution of a fixed point on the boundary: the case of $\mathbb{Q}$-rank one, in: The Zeta Functions of Picard Modular Surfaces, R. Langlands and D. Ramakrishnan, eds., Publ. C. R. M., Univ. de Montréal (1992), 465-478.

[Sa] L. Saper, Tilings and finite energy retractions of locally symmetric spaces, Comm. Math. Helv. 72 (1997), 167-202. 
[SaS] L. Saper and M. Stern, $L_{2}$ cohomology of arithmetic varieties, Ann. Math 132 (1990), 1-69.

[Sat] I. Satake, On compactifications of the quotient spaces for arithmetically defined discontinuous groups, Ann. Math. 72 (1960), 555-580.

[Sh] S. Shokranian, Lefschetz numbers on weighted cohomology for twisted groups, preprint, Dept. Matemática, Univ. de Brasilia, 1999.

[Sp] T. A. Springer, Linear Algebraic Groups, Progr. Math. 9, Birkhäuser, Boston, MA, 1981.

[St] M. Stern, Lefschetz formulae for arithmetic varieties, Inv. Math. 115 (1994), 241-296.

[Ve] J. L. Verdier, The Lefschetz fixed point formula in étale cohomology, Proceedings of Conference on Local Fields, T. A. Springer, ed., Lect. Notes Math. 192, Springer Verlag, Heidelberg (1971), 165-168.

[Vo] D. Vogan, Representations of Real Reductive Lie Groups, Progr. Math. 15, Birkhäuser, Boston, MA, 1981.

[Wa] G. Warner, Harmonic Analysis on Semisimple Lie Groups I, Grundl. Math. Wiss. 188, Springer Verlag, Heidelberg 1972.

[We] U. Weselmann, thesis, University of Bonn, 1996, http://www.mathi.uni-heidelberg.de/ weselman/.

[Z1] S. Zucker, $L_{2}$ cohomology of warped products and arithmetic groups, Inv. Math. 70 (1982), 169-218.

[Z2] S. Zucker, $L_{2}$ cohomology and intersection homology of locally symmetric spaces II, Comp. Math. 59 (1986), 339-398.

[Z3] S. Zucker, Satake compactifications, Comm. Math. Helv. 58 (1983), 312-343.

School of Mathematics, Institute for Advanced Study, Princeton, NJ 08540, USA e-mail: goresky@ias.edu

Eingegangen 25. Juni 2002 